\title{
Patient Mobility Monitoring through the Analysis of Bed Exit Centre of Pressure
}

by

\section{Zhaofen Ren}

A thesis submitted to the Faculty of Graduate and Postdoctoral Affairs in partial fulfillment of the requirements for the degree of

Master of Applied Science

in

Electrical and Computer Engineering

Carleton University

Ottawa, Ontario

(C) 2015

Zhaofen Ren 


\section{Abstract}

The increase in the older adult population and the benefits of independent living are leading to the introduction of new approaches for monitoring the health and well-being of seniors living in their own homes. Technologies that can monitor an older person's daily activities and detect changes in functionality have the potential to act as early warning systems so that help can be provided before a serious health event occurs. Home monitoring can contribute to the creation of more supportive environments for aging at home. This thesis uses a pressure-sensitive mat that is placed between a bed frame and the mattress. The pressure data can reveal important clinical information about the bed occupant. This thesis deals with the analysis of bed exit characteristics in terms of centre of pressure trajectory and its dynamic behaviour. The algorithms were tested on data collected from bed occupants to demonstrate the various clinical features. A graphical user interface (GUI) was designed to assist health care providers in interpreting and comparing these clinical features. This work will be used to monitor frail older adults in their own homes to assist with early detection of mobility decline. 


\section{Acknowledgements}

First and foremost I would like to thank my wonderful supervisors, Dr. Rafik Goubran, Dr. Mohamed El-Tanany and Dr. Frank Knoefel for their endless support and help throughout my research. They have been so patient and led me through the problems I faced during the last year not only at school but also in life. I would also like to thank the team members of the CIHR mobility project for the experimental data collection and for offering their expertise. Thanks to CIHR for the financial support.

Moreover, I am indebted to the help from Theresa Grant, Jordan Giberson and Stephanie Bennett for their help with language and proof reading of this thesis. I thank them for taking out time from their busy schedules to look over my thesis.

I love the working environment in the lab. Thanks to the students who are currently working or used to work in the lab to make such a friendly working environment. Especially I would like to thank Dr. Vilas Joshi, Mike Rockwood, Bruce Wallace and Matthew Taylor. Dr. Vilas Joshi taught me lots of things, answered my questions and made me feel so welcomed. I would like to thank them all for being a part of my journey and becoming an important part of my life.

Last but not least, I would like to thank my parents, without their tremendous trust and support I would not have come to Carleton University and do my master. Without them, I would not be who I am today. 


\section{Table of Contents}

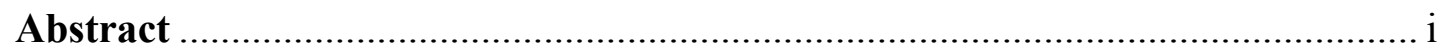

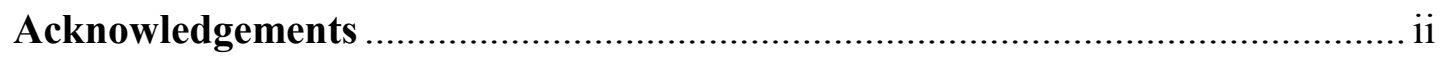

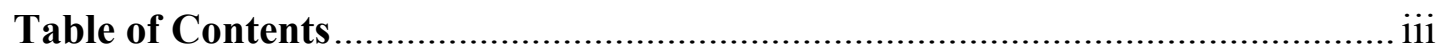

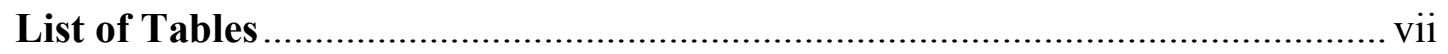

List of Illustrations ....................................................................................... vii

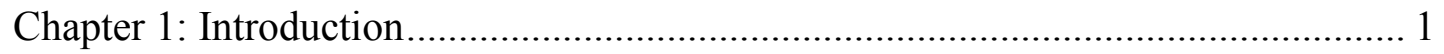

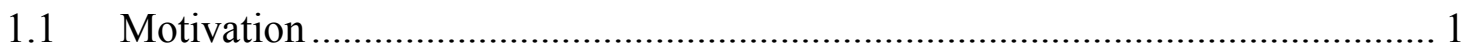

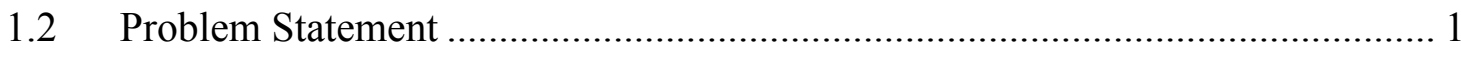

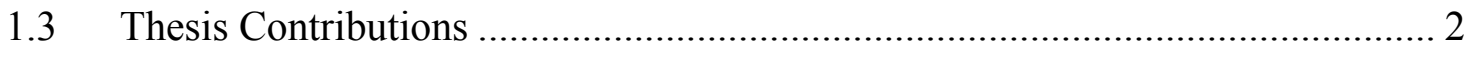

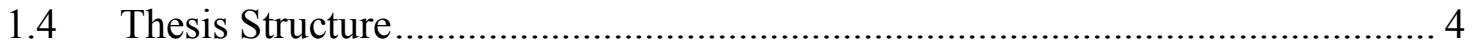

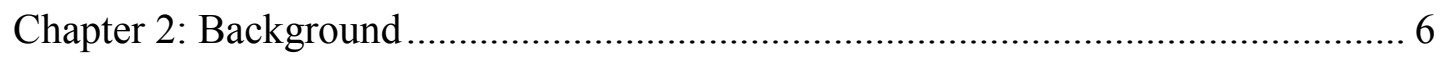

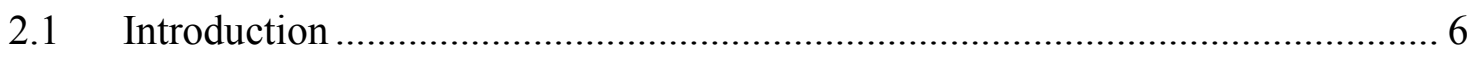

2.2 Technology

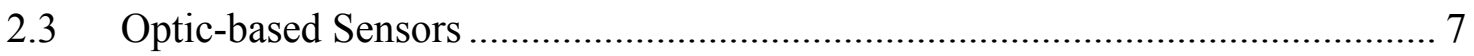

$2.4 \quad$ Related Work

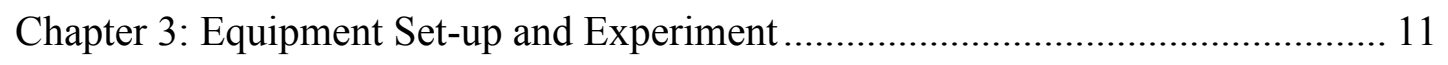




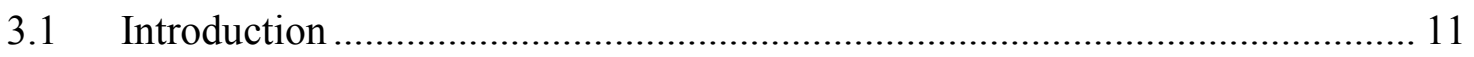

3.2 Equipment Set-up .............................................................................. 11

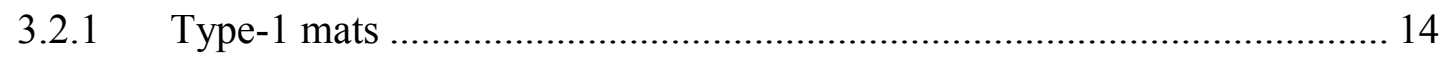

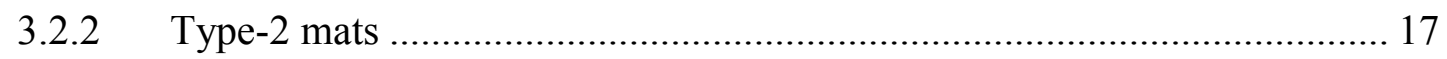

3.3 Volunteer Recruitment ................................................................... 21

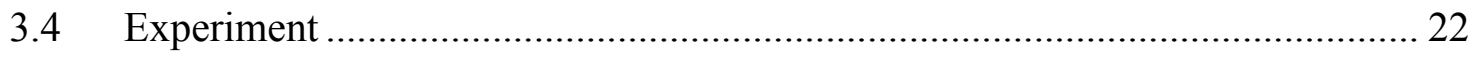

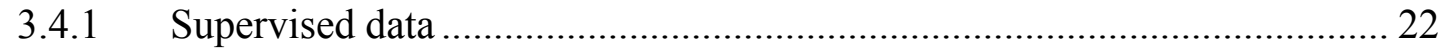

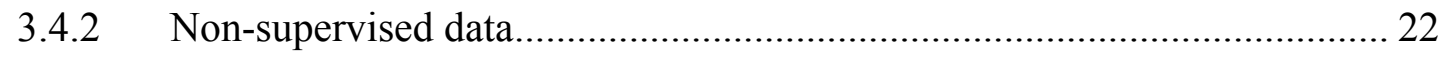

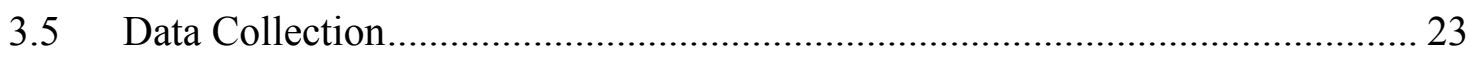

3.6 Data File Format................................................................................. 24

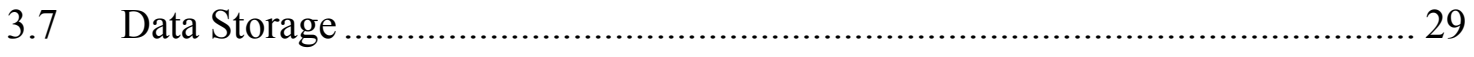

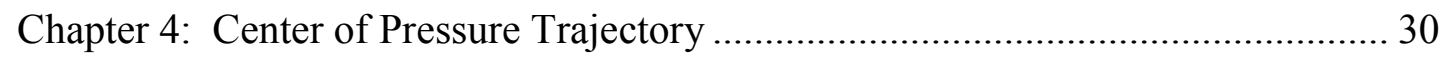

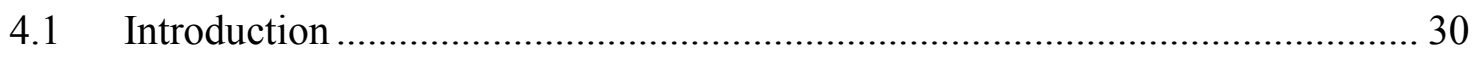

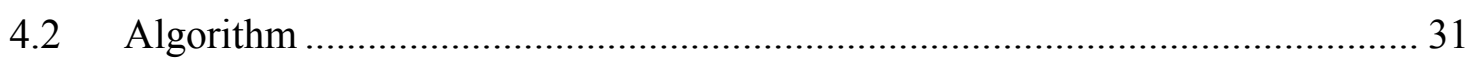

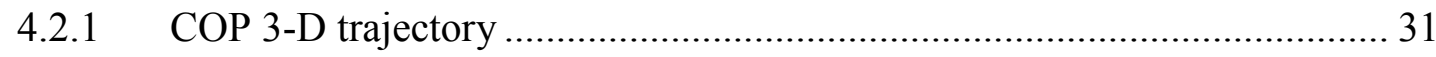

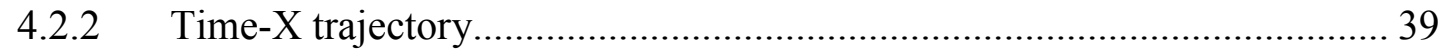

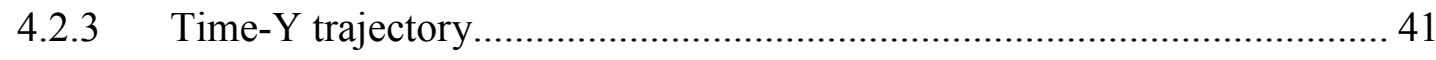

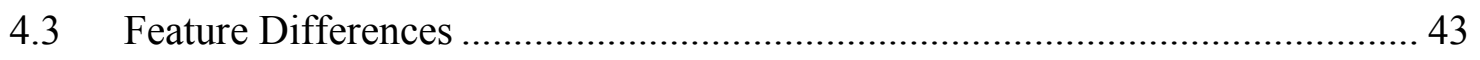

4.3.1 COP trajectory in 3-D graph difference before and after a fall ................ 43

4.3.2 Frequency color graph difference ................................................... 46 


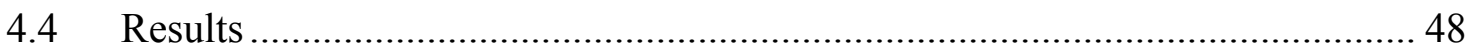

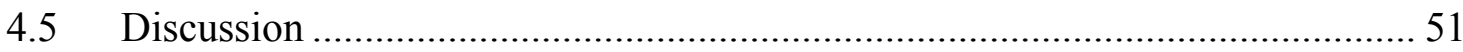

Chapter 5: Center of Pressure Magnitude................................................................... 53

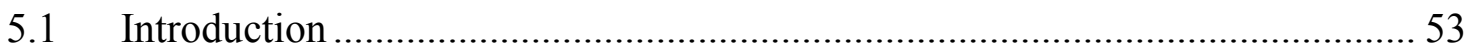

5.2 Generating Magnitude Graphs and Video...................................................... 54

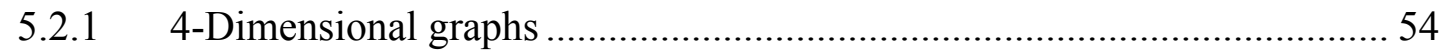

5.2.2 COP magnitude video ............................................................................ 56

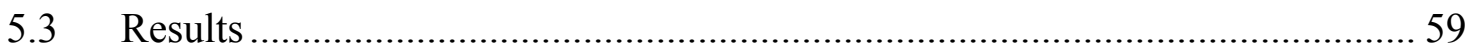

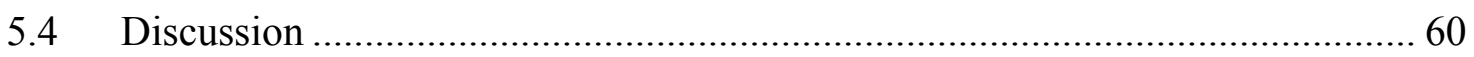

Chapter 6: Center of Pressure Dynamic Behaviour........................................................ 61

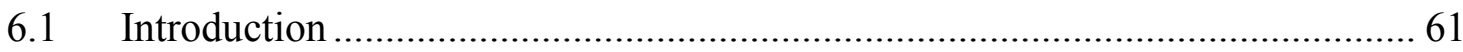

6.2 Algorithm for Calculating Velocity Magnitude ............................................... 62

6.3 Noise Suppression in the Computed COP Velocity Magnitude ......................... 67

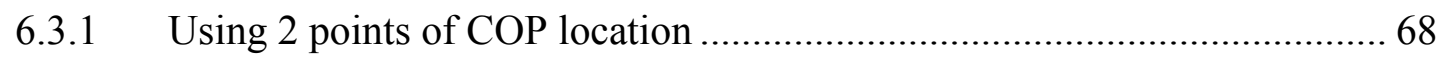

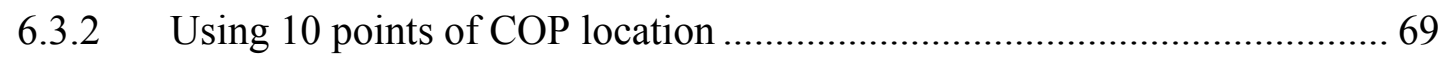

6.3.3 Using 20 points of COP location ............................................................. 73

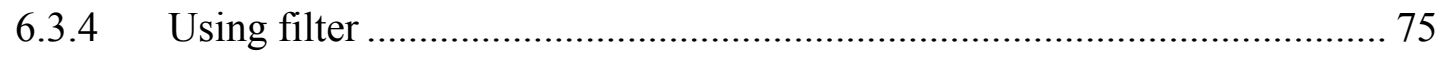

6.4 Algorithm for Calculating Rate of Change of Velocity Magnitude .................... 76

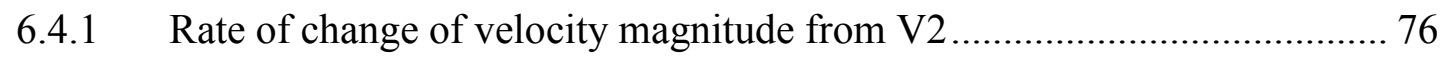

6.4.2 Rate of change of velocity magnitude from V10 ...................................... 77 


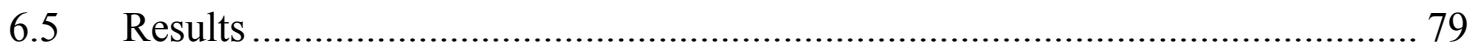

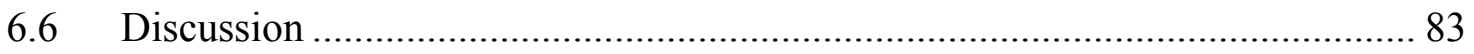

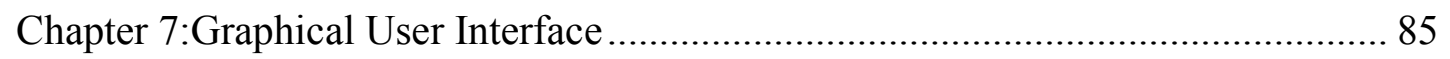

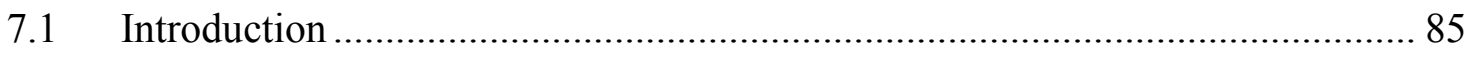

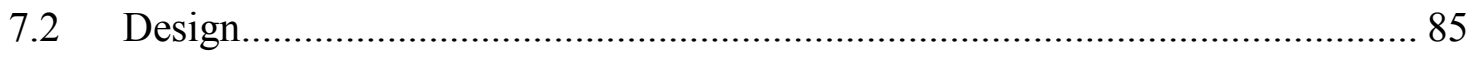

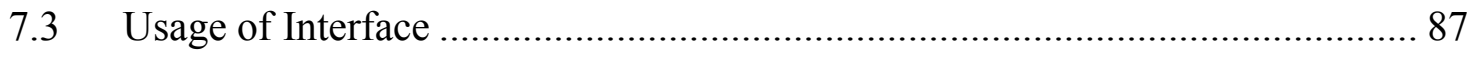

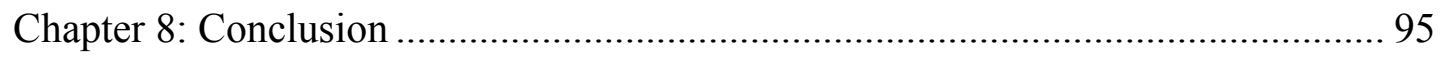

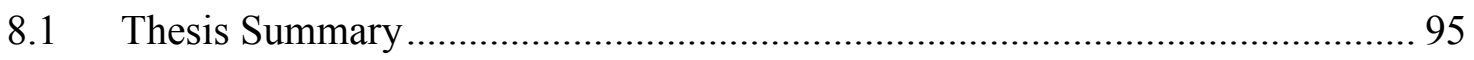

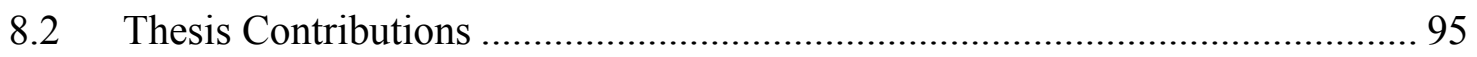

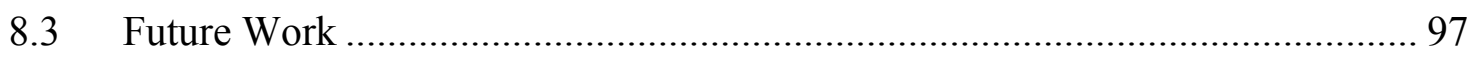

Reference 


\section{List of Tables}

Table 1: Type-1 mat data file sample ………………………................................... 25

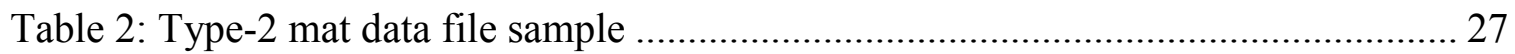




\section{List of Illustrations}

Figure 1: Optic-based pressure sensor structure. ......................................................... 8

Figure 2: Bed array install graph. ……………………..................................... 12

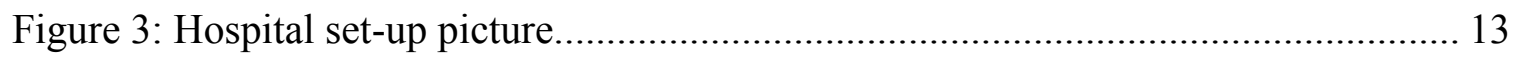

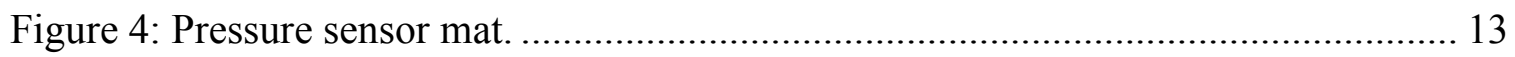

Figure 5: Type-1 mat inner sensor distribution diagram. ............................................ 14

Figure 6: Type-1 mat with connecting wire................................................................ 16

Figure 7: A recording box with Bluetooth to transmit data............................................ 17

Figure 8: Type-2 mat inside layout of sensors .......................................................... 18

Figure 9: One Type-2 mat with connecting wire ........................................................ 18

Figure 10: Type-2 mats signal sequence.................................................................. 19

Figure 11: Steps of building algorithm for Type-2 data files ........................................ 19

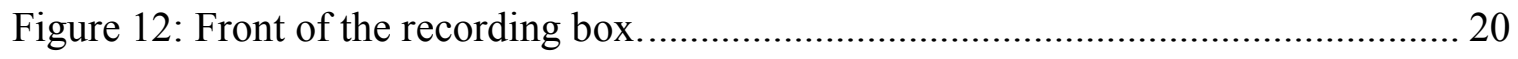

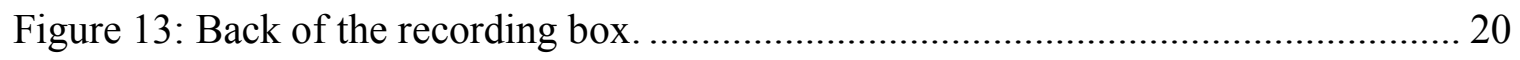

Figure 14: Type-1 mat sensor array, numbering system corresponding to txl column, table

1 .

Figure 15: Type-2 mat sensor array, labelled according to respective txl column, table 2.

(1)

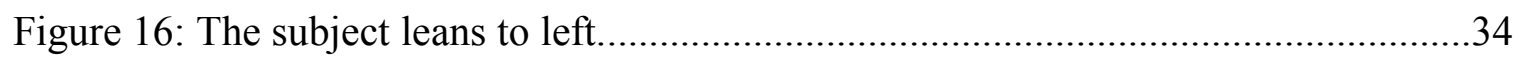

Figure 17: The subject leans to right.......................................................................... 33

Figure 18: time- $\mathrm{X}$ and time- $\mathrm{Y}$ trajectory plots versus time (in sample intervals) of lab test 1 
Figure 19: The COP 3-D trajectory plots of test 1 (used "Rotate 3D" function to make the

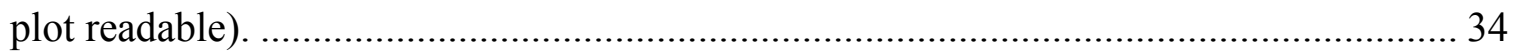

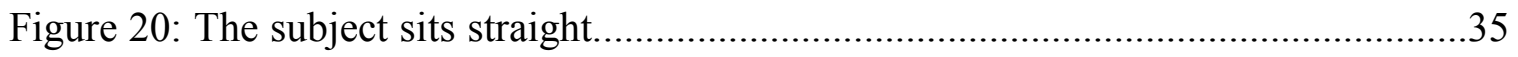

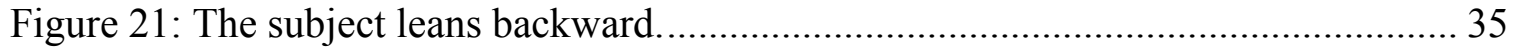

Figure 22: time-X and time-Y trajectory plots versus time (in sample intervals) of lab test 2 35

Figure 23: COP 3-D trajectory plots from test 2 (used "Rotate 3D" function to make the

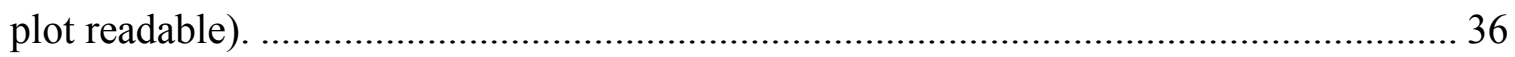

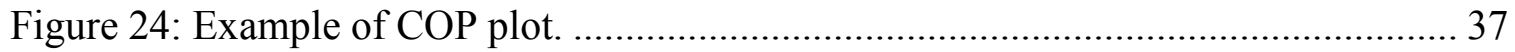

Figure 25: Color contrast plot showing the temporal variations of the Y-coordinate of the

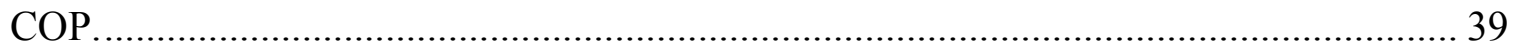

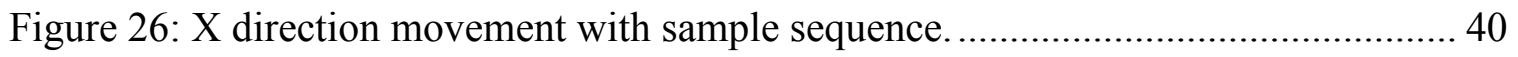

Figure 27: Time-X plot with real time and $\mathrm{cm}$ as unit for $\mathrm{y}$ axis................................... 40

Figure 28: Y direction movement with sample sequence. ..................................... 42

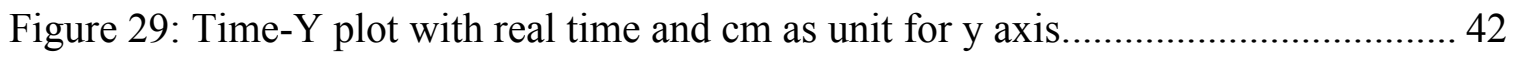

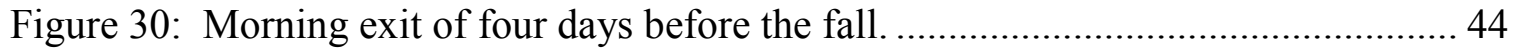

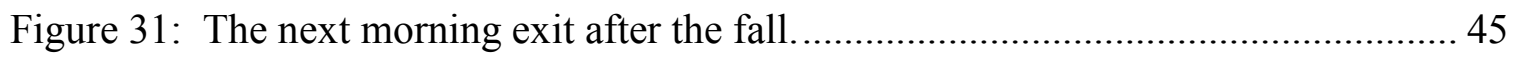

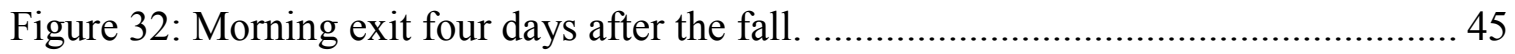

Figure 33: Color contrast plot of the morning exit four days before the fall showing the

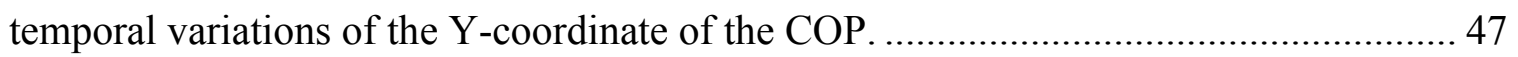

Figure 34: Color contrast plot of the next morning bed exit after the fall showing the temporal variations of the Y-coordinate of the COP. 
Figure 35: Color contrast plot of the morning exit four days after the fall showing the

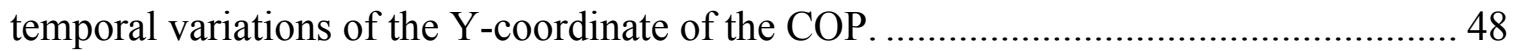

Figure 36: A young person getting out of the bed X direction plot.............................. 49

Figure 37: A young person getting out of the bed $\mathrm{Y}$ direction plot............................... 50

Figure 38: This young person bed-exit COP trajectory plot....................................... 50

Figure 39: Three young people break-down COP plots of bed-exit............................. 51

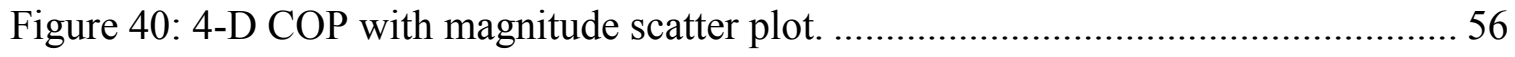

Figure 41: COP dot with a large pressure value (screenshot from a video). ................... 57

Figure 42: COP dot with a small pressure value (screenshot from a video).................... 58

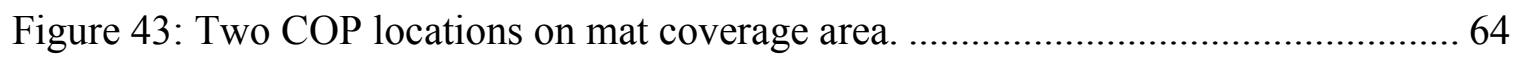

Figure 44: The subject leans to her left and her right with different speed for lab test of

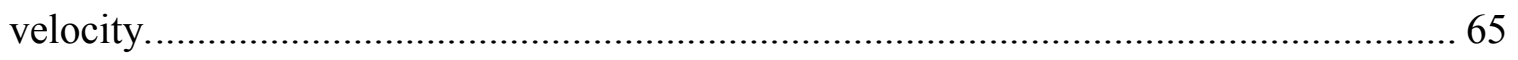

Figure 45: Slow and fast velocity plot from lab test showing on magnitude. ................. 66

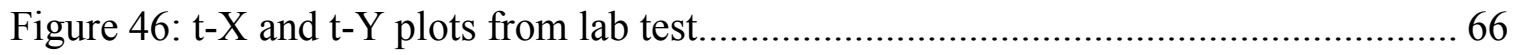

Figure 47: COP 3-D trajectory plots from test (used "Rotate 3D" function to make the

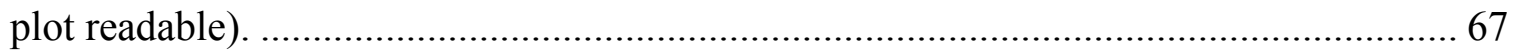

Figure 48: Velocity magnitude plot calculated by every two adjacent COP locations (V2).

Figure 49: Two ways to calculate $10 \mathrm{COP}$ points distance. ..................................... 71

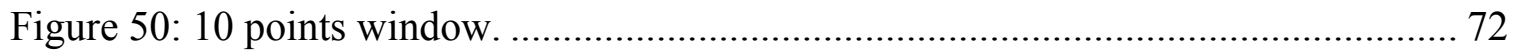

Figure 51: Velocity plot calculated using ten adjacent COP locations (V10)................. 73

Figure 52: Velocity plot calculated by twenty adjacent COP locations (V20)................ 74

Figure 53: Window 10 filtered velocity magnitude plot with V2............................... 75 
Figure 54: Window 20 filtered velocity magnitude plot with V2.............................. 76

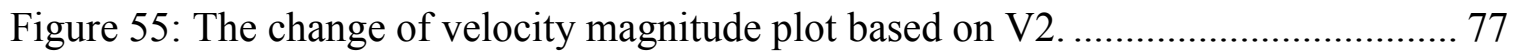

Figure 56: The change of velocity magnitude plot based on V10 ............................... 78

Figure 57: The change of velocity magnitude plot based on V20............................... 79

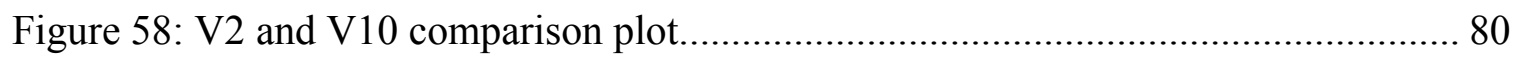

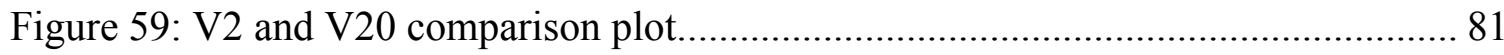

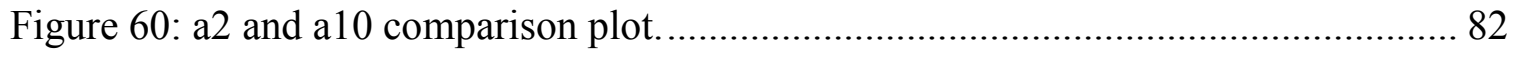

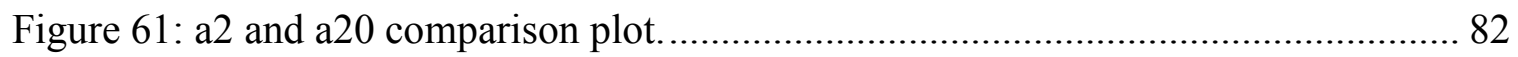

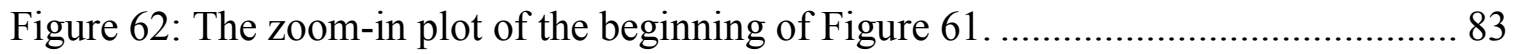

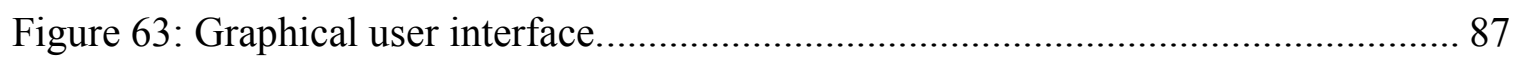

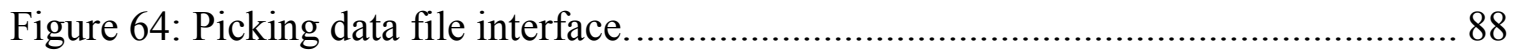

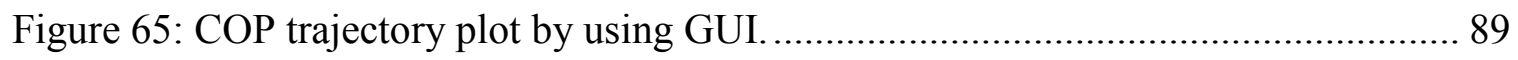

Figure 66: Comparison of two bed-exits on GUI. .................................................. 90

Figure 67: Choices of color for plots. ............................................................... 91

Figure 68: Sum of pressure changes during a bed-exit.......................................... 92

Figure 69: The number of active sensors during a bed-exit..................................... 93

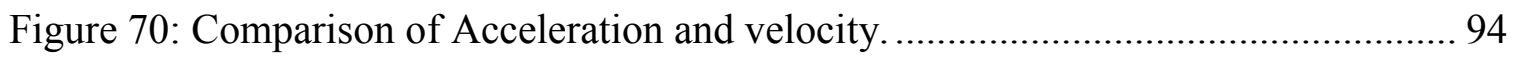




\section{List of Acronyms}

Act-Sen\#

ASCII

CIHR

COP

CSV

FIR

GUI

LED

LTS

LPF

LS

P.C.

SD

STS

Sum Pres

txl
The Number of Active Sensors

American Standard Code for Information Interchange

Canadian Institutes of Health Research

Center of Pressure

Comma Separated Values

Finite Impulse Response

Graphical User Interface

Light-emitting Diode

Lie to Sit

Low Pass Filter

Lying Stable

Personal Computer

Secure Digital

Sit to Stand

Sum of Pressure

Taxel - pixel in the pressure image 


\section{Chapter 1: Introduction}

\subsection{Motivation}

Over the last ten years, Canada's population has increased by 3.6 million [1], but the growth rate has recently declined [2]. The median age of Canada's population has increased, from 26.2 years old in 1971 to 39.9 years old by 2011, for a total increase in median age change of 13.7 years over 40 years [3]. By 2036, one in four Canadians will be 65 years or older [4]. The proportion of seniors in both the Canadian population and the world population is increasing, causing age-related problems; particularly economic and health related problems [5]. One particular problem resulting from this demographic shift is that large numbers of elderly people are living independently with a risk of mobility impairment and resulting injury. Common risks for mobility impaired seniors, and independently living seniors are falls, which are especially dangerous.

\subsection{Problem Statement}

With more older adults living at home independently, the need for monitoring is becoming more important. During hospital visits doctors perform medical tests on patients, and these tests may be enhanced by the information gained by daily monitoring. For example, a sudden loss of balance may be the forerunner of a stroke [6] and blood 
pressure drop with postural change may cause falls in old adults [7]. Daily monitoring of older adults has the potential to prevent such events.

The Canadian Institutes of Health Research (CIHR) has completed extensive research on many current and important matters [8], and now has an Institute on Aging dedicated to this research area. Some monitoring approaches require the use of wearable or implantable devices [9] whereas other approaches rely on sensors embedded in the environment to monitor home occupants such as the pressure mat used in this thesis. Bed pressure mats were investigated by many researchers [10]. Clinical features such as bed exit timing, symmetry and bouncing were considered. More clinical information needs to be extracted from long term monitoring data to help analyze individuals' health status and help predict falls. Long term data also needs to be collected from homes of older adults as opposed to only in-hospital data collection.

\subsection{Thesis Contributions}

In this thesis, under mattress optic-based pressure sensitive mats were used to collect pressure data of participants and then clinical features of bed-exit performance were extracted. The thesis contributions are listed as follows:

1. An algorithm was designed and implemented to convert the data from different mat types and mat configurations into a common format that can be processed with a single set of algorithms.

2. The second contribution was the design and implementation of several algorithms to measure the Center of Pressure (COP) location and the average 
pressure magnitude. These algorithms plot the COP trajectory and generate a video of the COP trajectory over time. The movements of a subject on the bed, including the sways and rocks during bed-exit transfers can be seen in the COP trajectory plot and video. This set of algorithms finally extracts clinical health information of a subject's mobility such as smoothness, symmetry and stability, which could help in fall prevention.

- [11] Z. Ren, T. Grant, R. Goubran, M. El-Tanany, F. Knoefel, H. Sveistrup, M. Bilodeau, and J. Jutai, “Analyzing center of pressure progression during bed exits," in Proc. IEEE 36th Annual International Conference of the IEEE Engineering in Medicine and Biology Society (EMBC), 2014, pp. 1786-1789.

3. The third contribution was to design and implement several algorithms to compute and plot the COP dynamic behaviour. The COP speed and its rate of change also revealed additional information about an individual's smoothness and timing of movements. Filtering was used to reduce the noise.

- [12] F. Knoefel, Z. Ren, T. Grant, M. Bilodeau, R. Goubran, H. Sveistrup, J. Jutai, "A method of analyzing bed exit movement in the home context," presented at Regional Geriatric Program of Eastern Ontario Annual General Meeting, Ottawa, Ontario, Canada, Oct. 24, 2014. 
- [13] S. Bennett, Z. Ren, R. Goubran, K. Rockwood, and F. Knoefel, "In-Bed Mobility Monitoring Using Pressure Sensors," submitted to 2014 IEEE Transactions on Instrument and Measurement, 2014.

4. The fourth contribution was the design and implement of a Graphical User Interface (GUI). This GUI allows the user to select any desired transfers from any patient. The user could then plot and compare any of the following features: COP trajectory, speed and its variation, average pressure magnitude, and the total number of active sensors. This GUI was written to plot multiple, super-imposed graphs of the same feature as well as plot two different features with two different scaling factors in one graph.

\subsection{Thesis Structure}

This thesis is divided into 8 chapters. The first chapter provides an introduction that describes the motivation behind this thesis, the problem statement, and thesis contributions. Chapter 2 gives the thesis background, including the technology used in the thesis experimentation, the working mechanism of sensors and existing research in the area of long term older adult monitoring. Chapter 3 explains the equipment set-up and discusses data collection from two types of mats and describes the file format. Center Of Pressure trajectory algorithms compare plots before and after a fall, and are described in Chapter 4. Chapter 5 discusses how the COP magnitude was calculated and the two ways of performing COP magnitude, providing 4 dimensional plots and video. Chapter 6 describes the calculation of COP speed and the rate of change, followed by noise reduction to make the plots clearer. Chapter 7 introduces the GUI that was designed for 
processing the data and presenting the results in a user friendly way. The last chapter provides a short summary and states the contributions of this thesis. 


\section{Chapter 2: Background}

\subsection{Introduction}

This Chapter provides the background information of the thesis. Section 2.2 and section 2.3 describe the technology that was used, and explain the operation of the optical based pressure sensor. Section 2.4 discusses existing research related to this thesis.

The concept of "smart homes" or "smart technology" has become popular in recent years [14]-[18]. Both young and old adults use the technology to make daily life easier, more comfortable and safer [19]-[21]. For instance, smart watches have been designed to detect gestures [22]. Smart phones have become medical diagnostic tools [23] and can be used to monitor activities of patients [24]. Smart TVs [25] and smart phones can remotely control home appliances at anytime from anywhere [26]. If hosts are not home, they can use their phone to turn off home appliances to avoid wasting energy and cause danger [27]. In many instances technology can help people who have health problems to monitor their health condition and improve their quality of life.

There is a growing interest in technologies that have the potential to remotely monitor aspects of an individual's evolving mobility status, so that critical changes may be detected early and more serious health problems prevented [28]-[30]. Getting out of bed is an activity performed by most people on a daily basis and also one that can be easily monitored using under mattress sensor technology. Difficulty with this common activity 
may be an important marker of physical decline since it clearly differentiates older adults living independently with those living in institutions [31].

This chapter describes the technology used in the thesis, the optic-based sensors working mechanism and related work done by others.

\subsection{Technology}

The technology used in the thesis, consisted of pressure sensitive mats which were used to collect data from volunteers. The data was transmitted by Bluetooth to a computer or saved on an SD memory card. MATLAB was used to process the data and obtain Center of Pressure (COP) trajectory and velocity. There were different types of pressure sensitive mats used in this research: Type-1 mat and Type- 2 mat systems that incorporate 72 optic-based pressure sensors but have different configurations. These differences will be discussed in Chapter 3. The technology provided a relatively unobtrusive way to monitor bed mobility in a continuous way [32].

\subsection{Optic-based Sensors}

The mats' sensors were optic-based pressure sensors made by S4 Sensors, Victoria, BC, Canada. Each bifurcated optic sensor was comprised of two plastic optical fibers, an emitter and a receiver [33]. The emitter and receiver strands were beside each other in a cellular foam structure, shown in Fig. 1 [33]. Light-emitting diode (LED) light was 
emitted through the emitter cable to the foam surface. When pressure was applied to a sensor, changing the pressure on the foam surface in turn changes the intensity of the light [34]. The light returns to a photo-diode through a receiver cable. The photo-diode converts the light to an output voltage which varies with the pressure being used on the sensor [34].

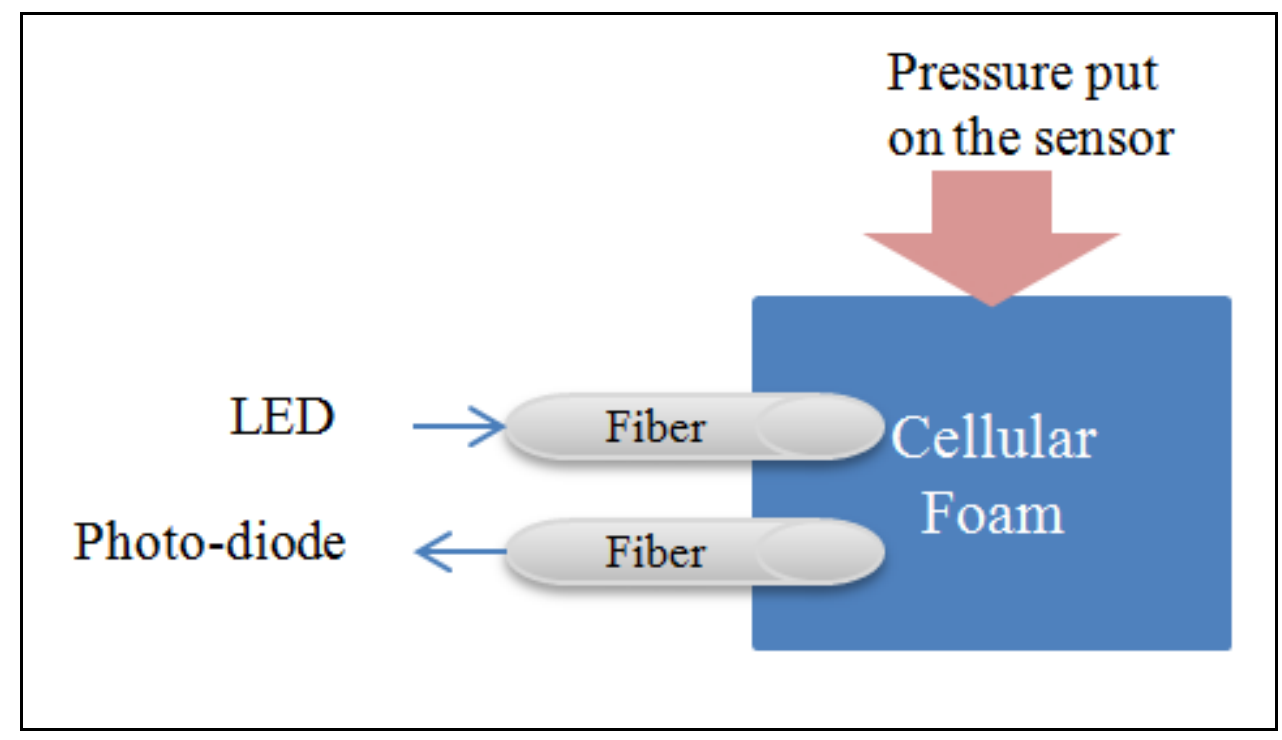

Figure 1: Optic-based pressure sensor structure.

Optic-based sensors do not contain any moving parts or electrical circuitry. Therefore, electrical interference is not a problem. The sensors can be used in different environments and without danger to the user. For these reasons as well as the low manufacturing cost, optic-based sensors are widely used [35].

\subsection{Related Work}

Sensor technologies are being developed as a part of home monitoring systems [29], [30]. Researchers devoted considerable time to "smart home" technologies which can automate 
or remote control home electronics to provide people a secure and comfort home. Radar [36], [37] and Kinect have been used to monitor movement patterns in the home for the purpose of determining an older individual's fall risk [38], [39]. Pressure sensors have been used to monitor force distribution related to grab bar use [29] as well as breathing patterns related to sleep apnea [40].

Under mattress pressure sensor technology has been used to monitor various aspects of bed mobility including sit-to-stand time [32], [33], [41], bouncing [42], symmetry [41], classification of bed movements [43] and bed occupancy [10]. Many students conducted research on pressure sensitive mats at Carleton University. Daphne Townsend validated the use of unobtrusive mats for central sleep apnea screening [44] and proposed feature extraction algorithms for detecting central sleep apnea [45]. She also designed adaptive signal processing algorithms to measure torso movements [46]. Amaya Arcelus designed algorithms to measure bed transfer characteristics such as sit-to-stand transfer duration [32], symmetry [41] and bouncing [42]. She also explored the use of a pressure-sensitive floor plate to better characterize bed exits [32] and to measure the standing stability of patients [47]. Signal processing algorithms were proposed to measure the breathing characteristics of a bed occupant [48] including respiratory disturbances [49] and respiratory effort [50]. The results revealed that detecting the lying posture is important in optimizing the parameters of the breathing detection algorithms. Nick Foubert and Anita McKee designed the appropriate feature extraction and classification algorithms to perform this function [51]. Stephanie Bennett proposed an algorithm for the automated assessment of mobility in bedridden patients [52]-[54] and was able to distinguish 
between stable and unstable sit-to-stand transfers using pressure sensors [55]. Additional work was conducted this year to monitor motion in palliative care [56].

Work completed by Amaya Arcelus [57], focused on context-aware monitoring using unobtrusive pressure sensors. Pressure sequences were analysed for Sit to Stand (STS) transfers through under a mattress mat and on the floor mat [32], [33], [41]. The COP trajectory and the duration of STS were used to classify impairments and separate healthy people from mobility-impaired people [58]. Matthew Taylor processed pressure images of bed mat and floor array to extract clinical features [59]. He processed floor sensors' data to get pressure images compared stability between a young person and an older adult [47], [60] and obtained individuals bed-exit time from bed mat [61].

This thesis proposes the use of center of pressure features to analyse bed exits. The data is collected from pressure sensitive mats which have different layouts of sensors. The center of pressure data reveals important information about how an individual gets out of bed. This information has not yet been studied with under mattress pressure sensor technology in the home context. 


\section{Chapter 3: Equipment Set-up and Experiment}

\subsection{Introduction}

This chapter describes the equipment set up and experimental methodology. This chapter also discusses differences between the two types of mats and how the experiment was conducted. Sensors have been used in the home context in many ways for some time [18] such as sensors that were installed in television sets (Infrared sensors at remote control to issue commands), water kettles (temperature sensors to control the temperature of water) and smart lamps (passive infrared sensors to detect motion to turn on and off the light). Widespread use of sensors has contributed to growing acceptability for use in long term monitoring of daily routines.

\subsection{Equipment Set-up}

The technology used in this study consisted of a pressure sensitive mat array composed of 72 fiber optic pressure sensors with high sensitivity to pressure changes [34]. The pressure sensors were equally distributed in a grid structure. The entire mat covered an area of approximately 80 by 90 centimeters. 
The pressure sensitive mat array was placed under the bed mattress and was connected to a transmitter box or a recording box. The transmitter boxes transmitted the information to a computer via a Bluetooth connection. The recording boxes saved the data on a secure digital (SD) card. A computer connected to a transmitter box ran a custom Windows XP system. Both transmitter box and computer were placed under the bed. We designated the pressure sensitive mat's $\mathrm{X}$ axis to correspond with bed width while the mat's $\mathrm{Y}$ axis corresponded to the bed length. This orientation scheme is illustrated in Fig. 2.

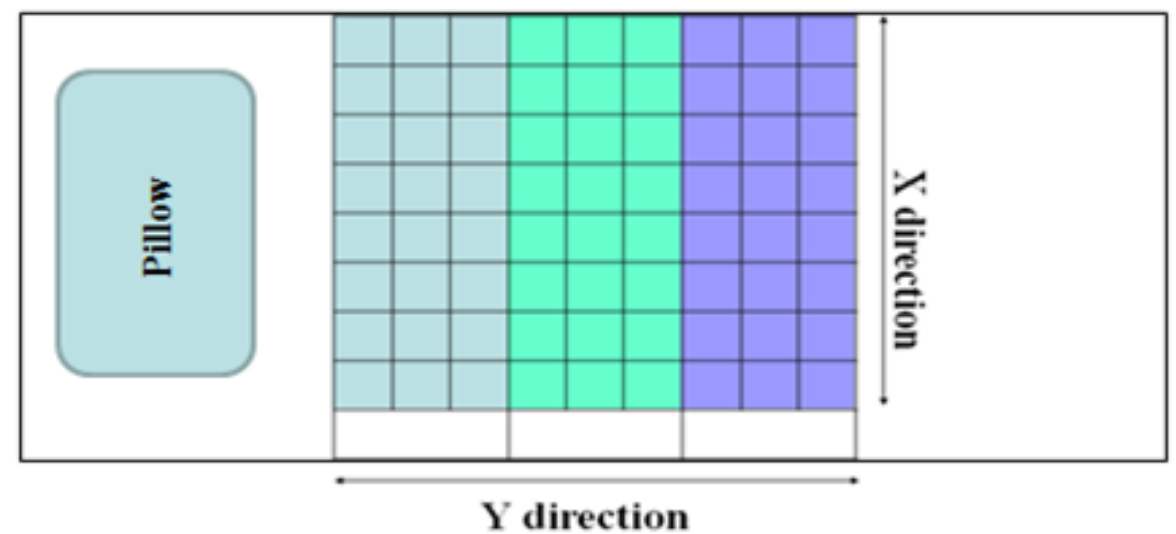

Figure 2: Bed array install graph.

The pressure mat systems were designed to accommodate standard home beds as well as hospital beds. For hospital beds, the mats were taped securely to the solid bed frame and the mattress sat on top. This configuration is shown in Fig. 3, Type-1 mat is in between of the mattress and bed frame (black).

In the home environment, mats were first taped securely to a plywood board which provided a stable surface between the box spring and mattress. If there was no box spring, the pressure sensitive mat is put on the top of bed frame underneath the mattress. Figure 4 is a lateral view of the home set up. In the case of community participants, 
systems were left in place for up to 12 months and data was collected on a continuous basis.

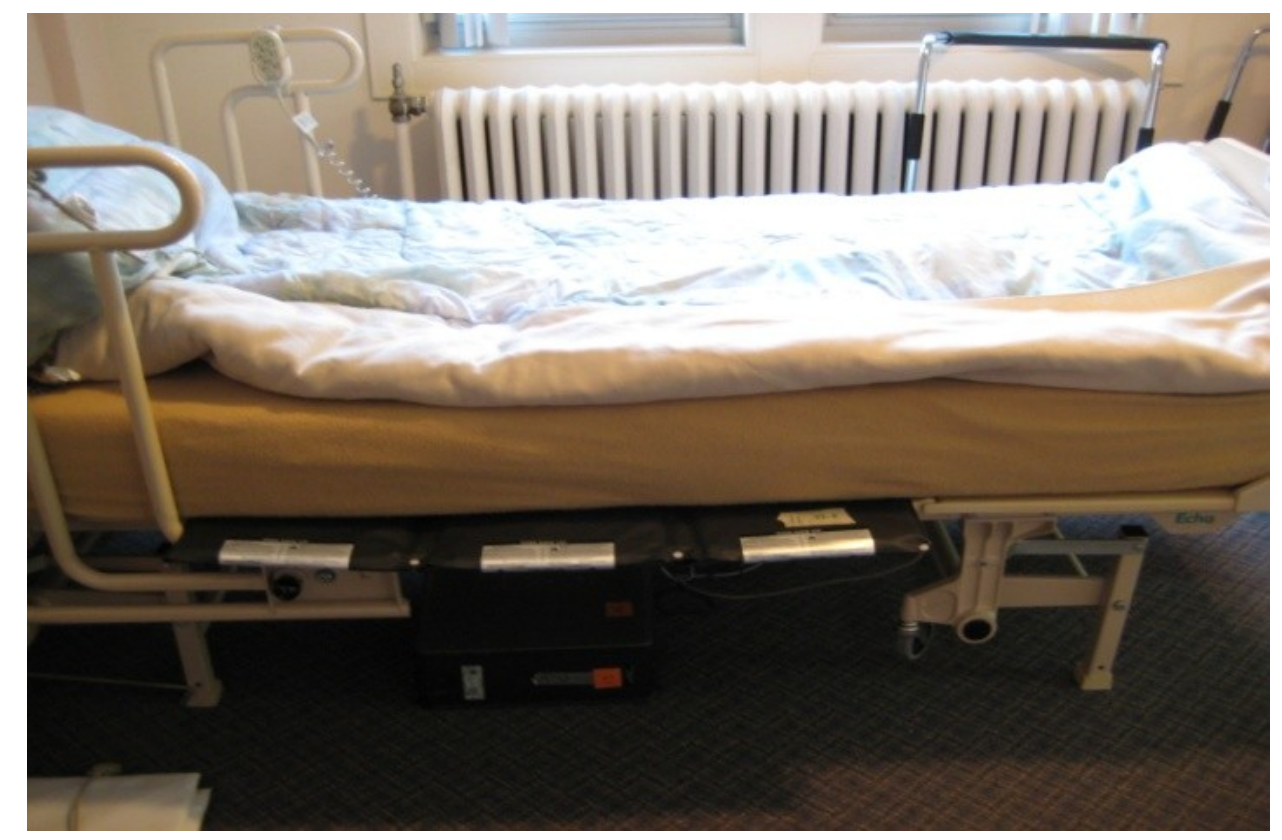

Figure 3: Hospital set-up picture.

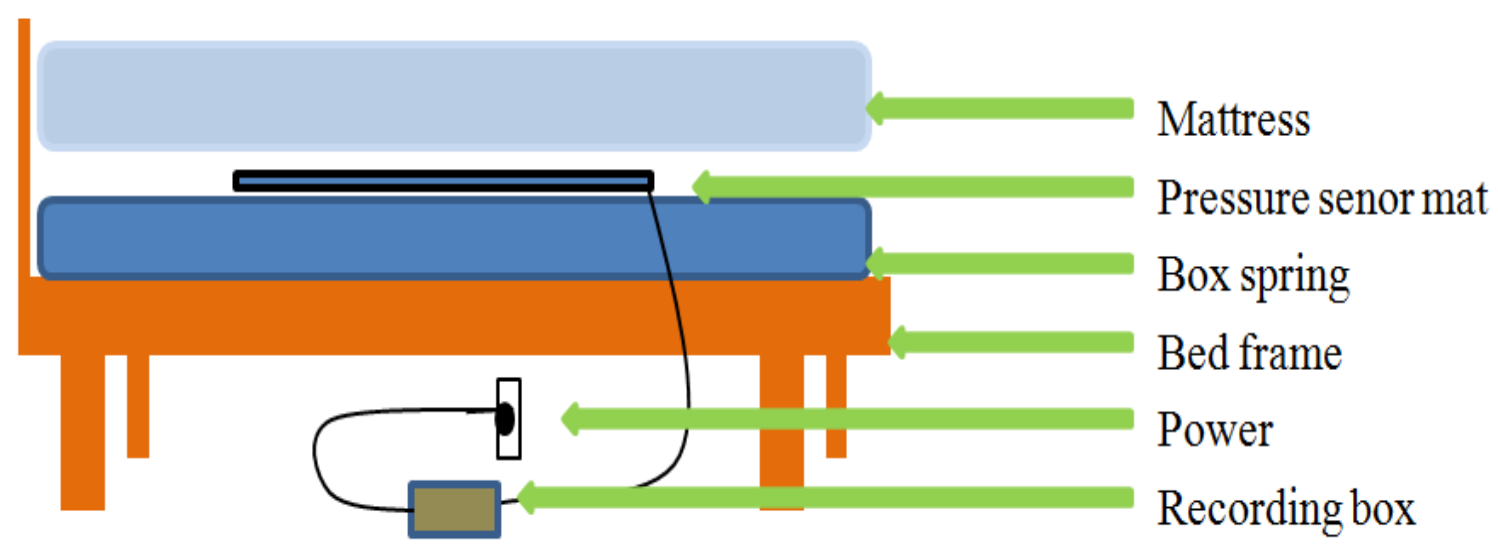

Figure 4: Pressure sensor mat. 


\subsubsection{Type-1 mats}

The Type-1 mat was composed of three panels as one piece containing 72 sensors in total. The 72 sensors were distributed evenly in the mat, as Fig. 5 shows. Sensors were embedded in foam as an 8 by 9 matrix, with 8 sensors lining the width and 9 sensors lining the length of the mat. A mat head exits in each panel of the pressure sensor array, located on the long side containing mat associated hardware.

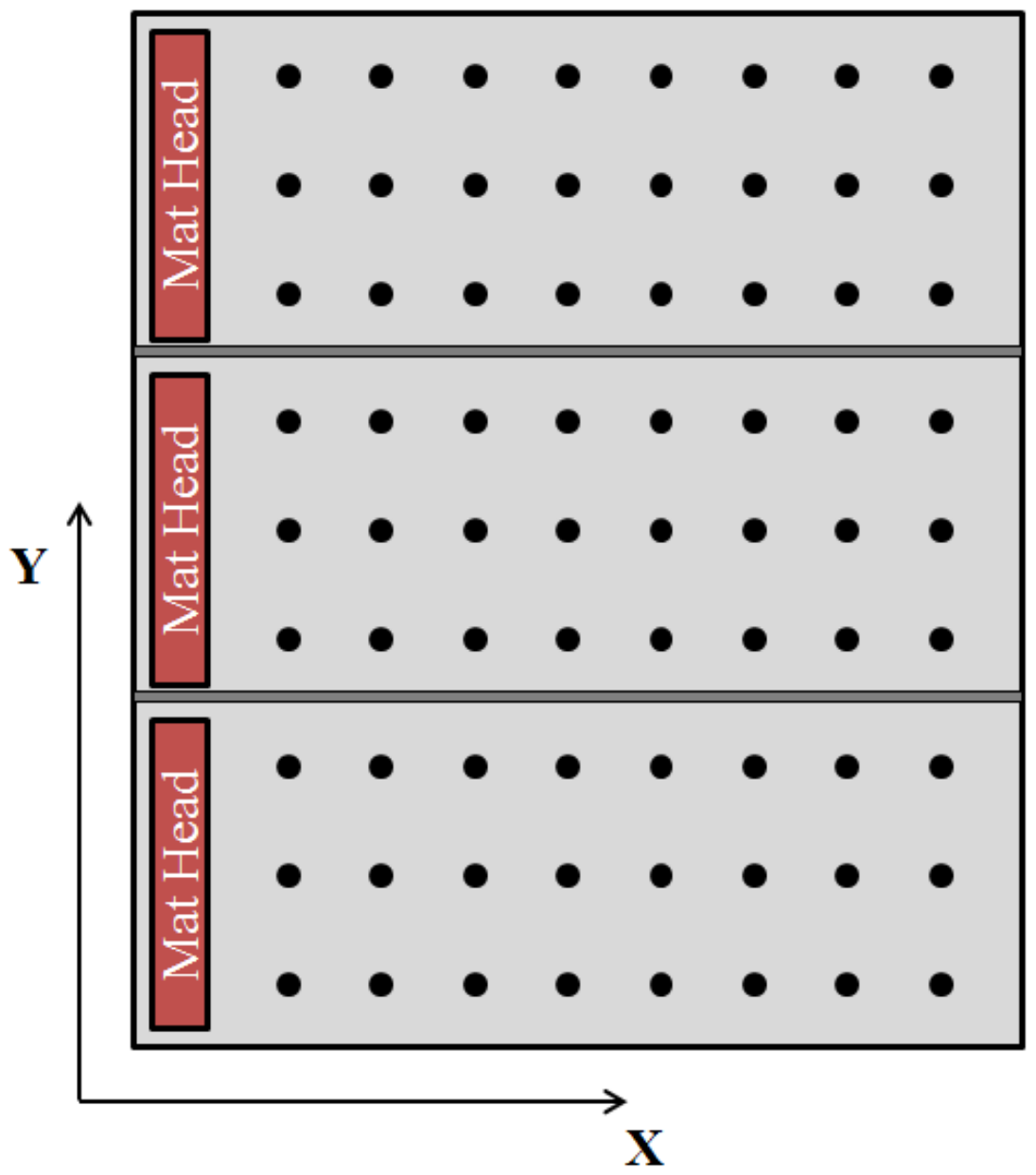

Figure 5: Type-1 mat inner sensor distribution diagram. 
Figure 6 is a picture of the Type- 1 mat with connecting wire. Figure 7 is a picture of the logger box used to connect with Type-1 mat. Type- 1 mats could work with the Type- 2 mat's logger box. However, in most systems we used transmitter boxes as the one shown in Fig. 7 with the Type-1 mat. One logger box and power supply was needed to connect with the Type-1 mat to collect data. Those data were sent to a server via Bluetooth or auto-saved as comma separated value (CSV) files on an SD card, depending on which recording box was being used. Data from 72 sensors were collected simultaneously with a sampling frequency of $20 \mathrm{~Hz}$. Data file size and format were different between Type-1 mats and Type-2 mats which will be discussed in sections 3.5 and 3.6. 


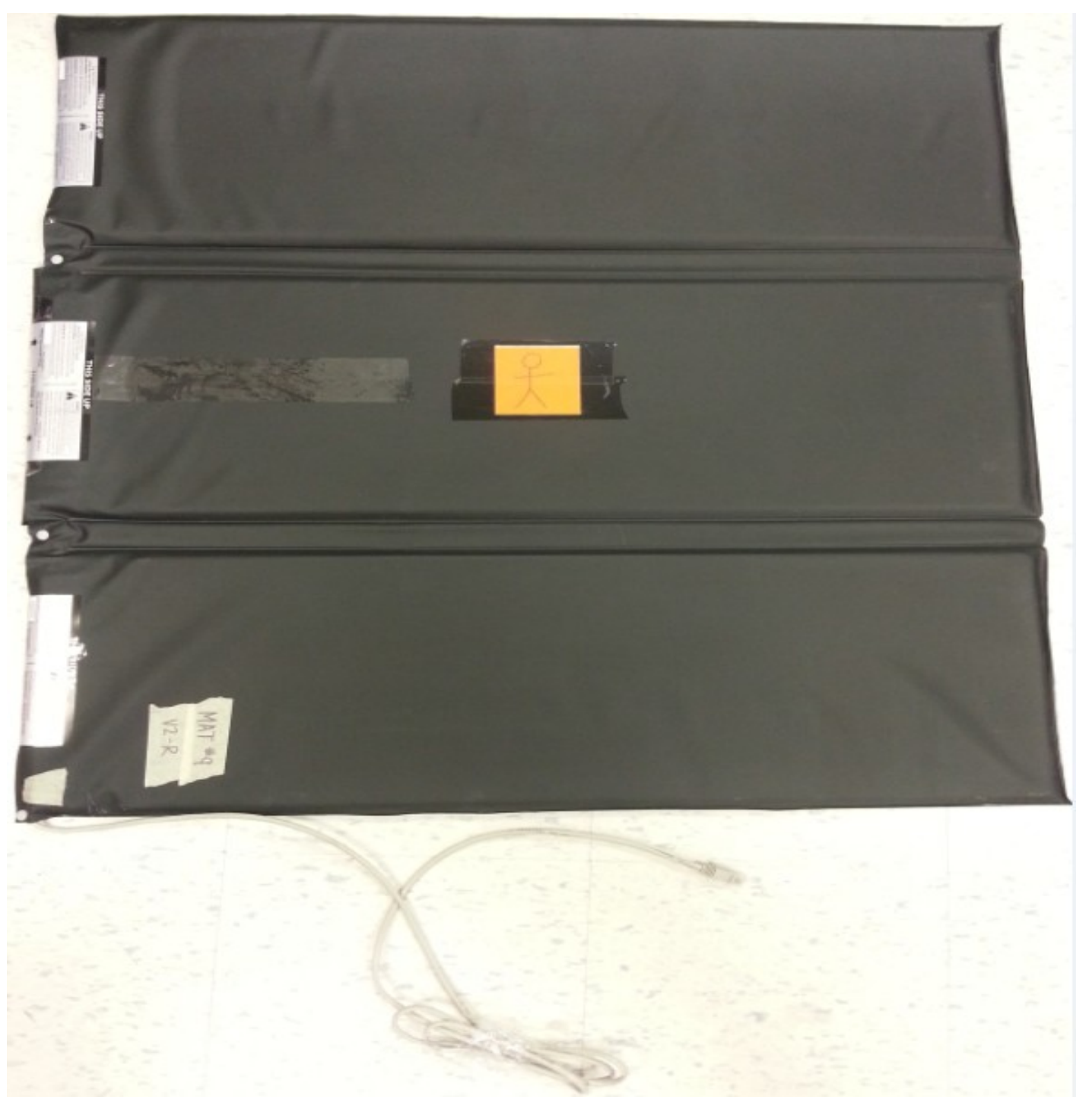

Figure 6: Type-1 mat with connecting wire. 


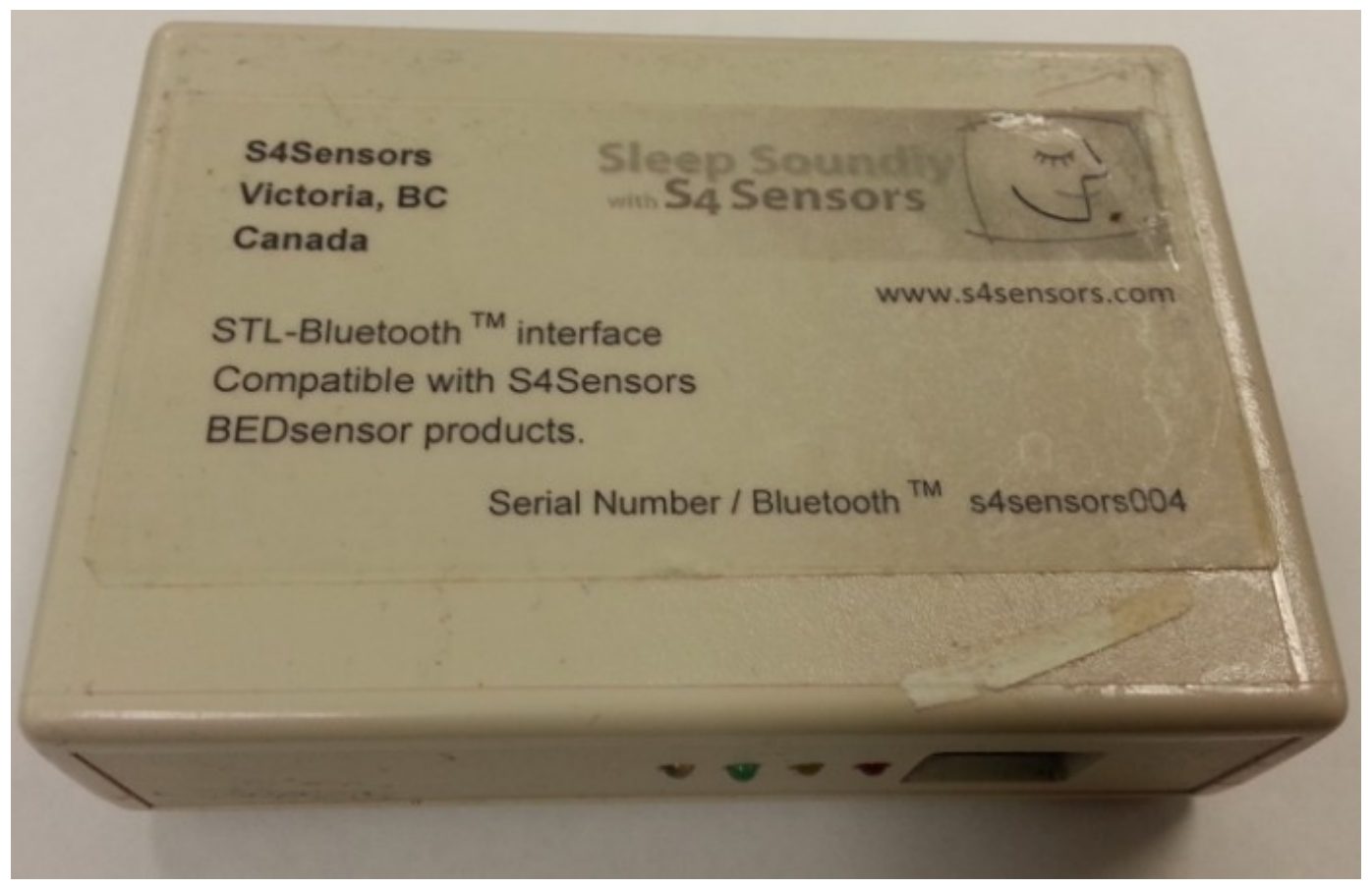

Figure 7: A recording box with Bluetooth to transmit data.

\subsubsection{Type-2 mats}

Type- 2 mats were composed of three separate panels. Each panel consisted of an 8 by 3 pressure array. The side with 8 sensors was designated as direction $\mathrm{X}$ corresponding to the short side of the bed. The side with 3 sensors was designated as direction Y, corresponding to the long side of the bed. One set of Type- 2 mats had 72 sensors which was the same number of sensors contained in Type-1 mats. Sensors in Type- 1 and Type- 2 mats had the same functions. Figure 8 shows the sensor lay-out structure of one piece of pressure sensor array panel. There was a slightly raised rectangular part on one side of the panel, which contained relevant hardware. Figure 9 is a picture of the mat panel. 


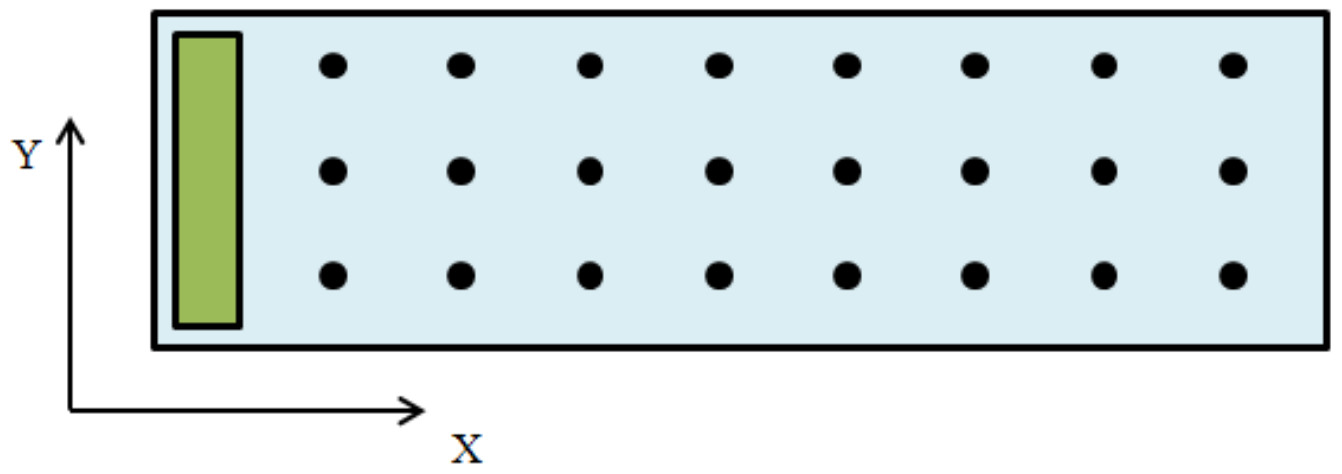

Figure 8: Type-2 mat inside layout of sensors

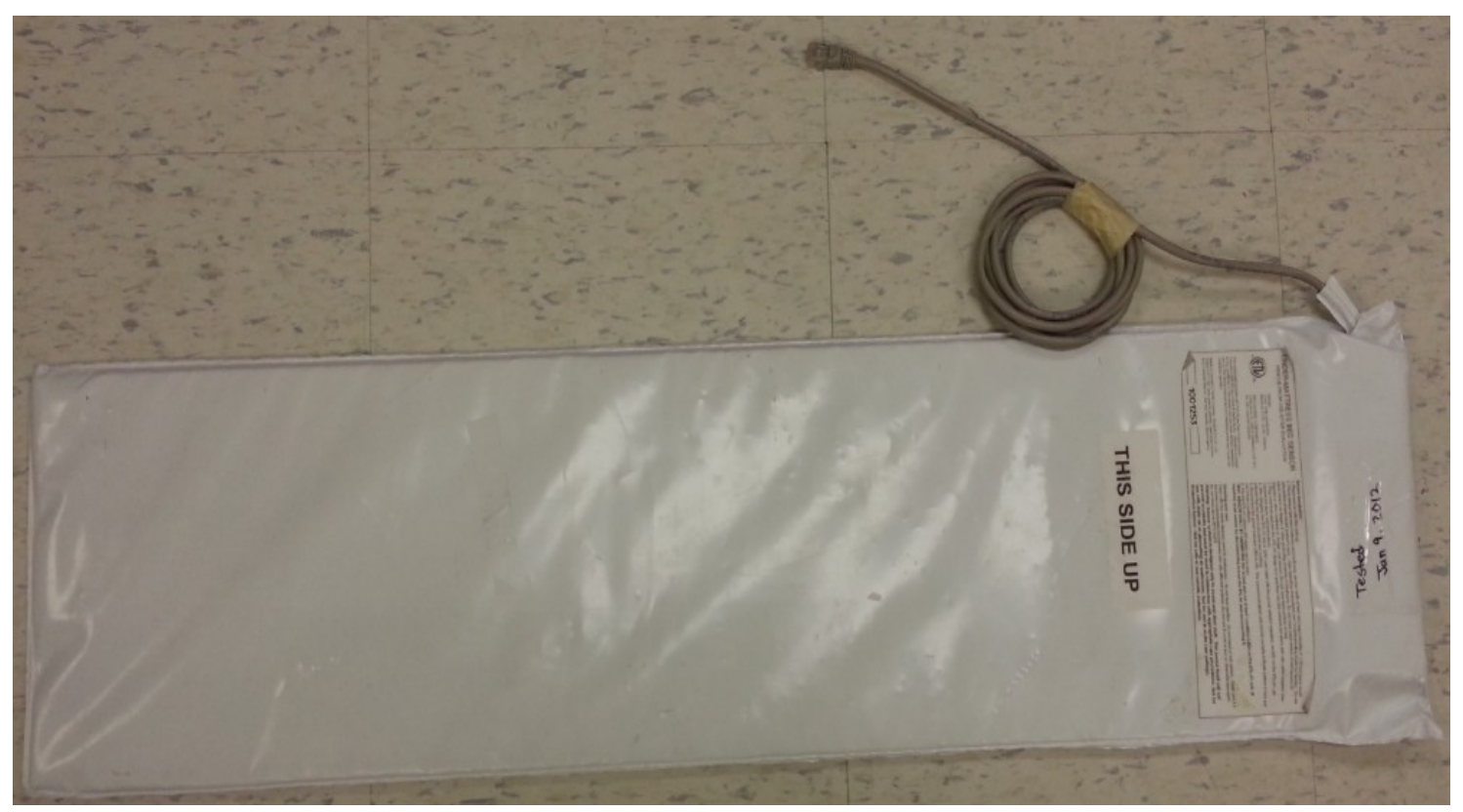

Figure 9: One Type-2 mat with connecting wire. 


\section{Type-2 mats}
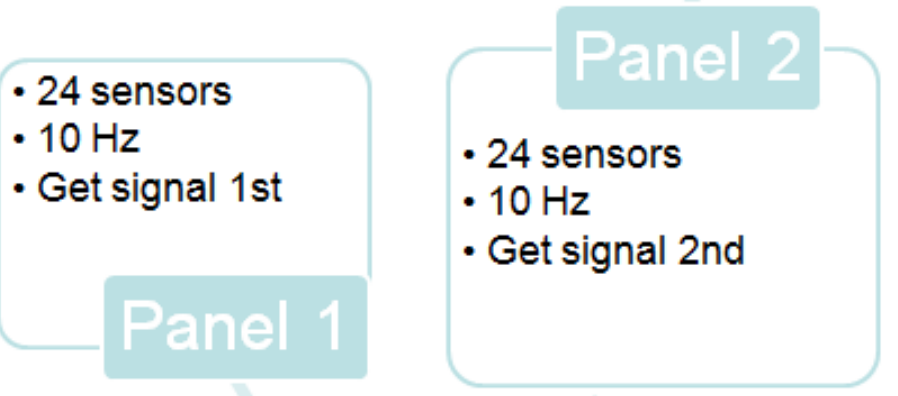

- 24 sensors

- $10 \mathrm{~Hz}$

- Get signal 3rd

Figure 10: Type-2 mats signal sequence.
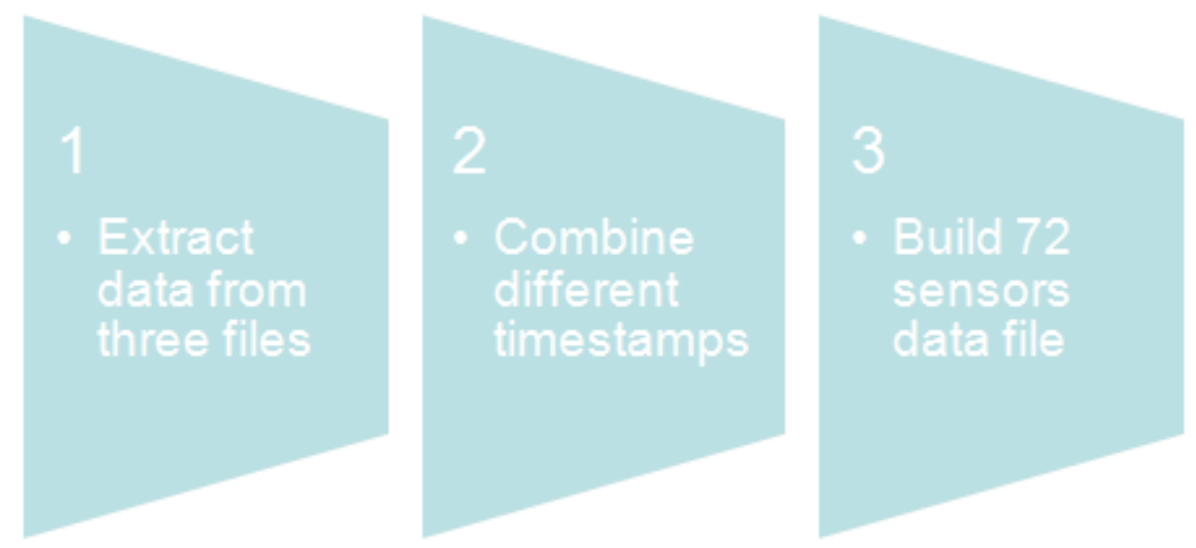

Figure 11: Steps of building algorithm for Type-2 data files

Three Type- 2 mat panels connected with one recording box to collect data at a sampling frequency of $10 \mathrm{~Hz}$. The recording box saved CSV files of data in hourly intervals. The logger box sent a signal to the first panel to obtain data to write into a data file. The logger box then sent a signal to the second and third panels to obtain data from each of those to be written onto separate files, as shown in Fig. 10. This process was repeated 10 times every second. All the CSV files were saved on an SD card. Every hour there were 
three separate files created for each set of the Type-2 mat panels. Since the signal logger box sent signals to the three mat panels, one after the other, there was slightly different timing. This meant timestamps on data files from the three mat panels were accordingly different. The recording box saved data files and files names separately and differently. That allowed the researchers to know which files were associated with which mat panel according to how they were labelled. Figure 12 and 13 show the front and back panels of the recording box. The front of the recording box had three ports which connected with sensor panels and three lights which indicated whether the three panels were plugged in, whether they were working and whether the box was turned on. An on/off button, SD card slot and a power port were located on the back panel.

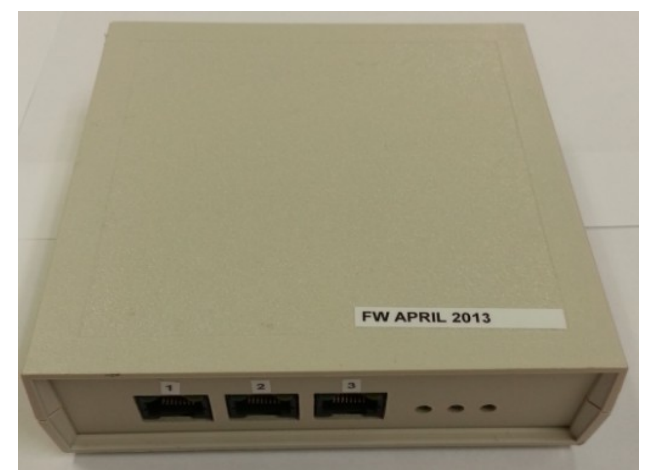

Figure 12: Front of the recording box.

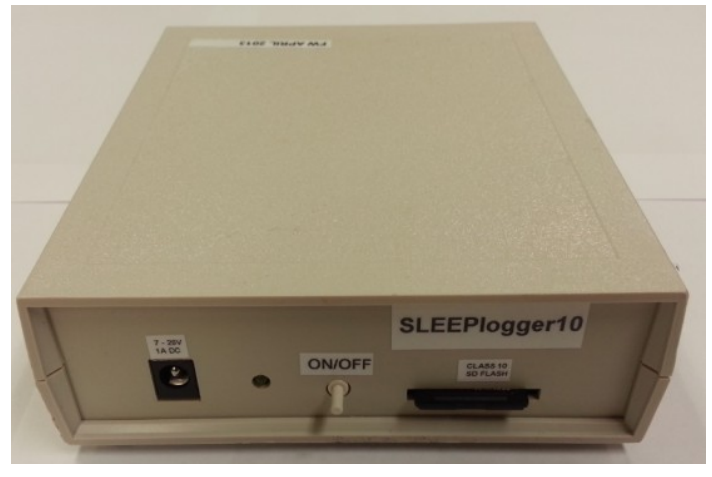

Figure 13: Back of the recording box. 


\subsection{Volunteer Recruitment}

The algorithms that were proposed and designed within the scope of this thesis are intended to be used for processing the data that is currently being collected by our clinical research partners at the Élisabeth Bruyère Hospital. This section describes the protocol used for data collection. A physiotherapist from Élisabeth Bruyère Hospital introduced and explained this project to potential volunteers at hospital and social gatherings. Those who were interested and meet inclusion criteria (65 years of age or more female/male, community dwelling older adults living in affordable seniors housing, and patients admitted to Geriatric Rehabilitation Unit at Élisabeth Bruyère Hospital) signed consent forms before participating in the project. This process recruited around 50 senior volunteers from different communities. There were 25 sensor systems set-up in Élisabeth Bruyère Hospital beds after patients agreed and signed consent forms. As stated previously, systems were installed in 25 seniors' homes for long-term monitoring (monitoring time was 8-12 months) and in Élisabeth Bruyère Hospital patient hospital beds for short-term monitoring (monitoring time was 1-4 weeks) during the patients stay. The recruitment of volunteers took place over a two year period.

Five young volunteers, all in their 20's, performed bed-exits in the digital signal processing laboratory at Carleton University to collect some reference data to design and test the algorithms. 


\subsection{Experiment}

\subsubsection{Supervised data}

Every month the physiotherapist visited the community-dwelling participants' homes and asked participants to get into and out of bed three times as they would normally do in the morning. These three bed transfers were video-taped monthly. Participants started by lying down on the bed and then exiting and coming into a standing position. Supervised data with video is gold standard. Having video information helped to assist with the interpretation of pressure-sensitive mat data since information obtained from mat technology could be cross-referenced with video information.

In a laboratory at Carleton University, five young healthy participants did similar transfers that involved lie-to-sit (LTS), and sit-to-stand (STS) phases using the same technology to monitor these transfers. Younger people performed their transfers under controlled conditions. This allowed for the comparison of younger movement patterns with those of older people.

\subsubsection{Non-supervised data}

The systems installed in the community were left for up to a year to continuously collect pressure data. Except for one visit every month in which transfers were supervised, the rest of data was not supervised. Since systems were collecting data in the real life context there was more noise and uncertainty factors to affect the authenticity and veracity of 
data than there would be in a laboratory setting. For instance, if a pet jumps on the bed or visitors sit on the bed, data can appear to be irregular. Notes were kept when possible on the occurrence of these events when known. In addition, home environments can be varied when participants move their bedroom furniture or unplug the technology system by mistake. All these factors present extra challenges to collecting data in the home environment compared to the laboratory environment.

While lab controlled data may reflect higher accuracy in some ways, data collected in the home environment reflects the realities of daily life that must be considered if home monitoring technologies are to be relevant in the future.

\subsection{Data Collection}

Data were collected in ASCII format and auto-saved as CSV files every hour. For Type-1 mats, each hour had one large CSV data file, and for Type-2 mats each hour has three smaller files. One hour Type-1 mat's file was about 21 MB. However, one hour Type-2 mat's file was only approximately $4 \mathrm{MB}$ owing to each file containing data from one panel. One panel had 24 sensors functioning at a frequency of $10 \mathrm{~Hz}$. That was half of Type-1 mat's $20 \mathrm{~Hz}$ frequency. For one participant who was using a Type-1 mat, approximately $500 \mathrm{MB}$ of space is needed to store a day of data, so that after one year there is 175 GB data. For a participant who was using a Type- 2 mat, approximately 288 $\mathrm{MB}$ of storage space is required for one day, so that after one year there is $100 \mathrm{~GB}$ data saved. 
$500 \mathrm{MB} \times 360=180000 \mathrm{MB} \approx 175 \mathrm{~GB}$

$288 \mathrm{MB} \times 360=103680 \mathrm{MB} \approx 100 \mathrm{~GB}$

Older participants had the systems collecting pressure related bed mobility data unobtrusively during this study. They were also asked to record any falls and critical health events they had over the study period. This information was collected monthly along with the pressure data by the physiotherapist during the monthly visits. Clinical measures of physical function were done during these monthly visits as well.

In this thesis, most of the plots were obtained from processing data files of Type-1 mats. The plots from data files of Type- 2 mats will be noted because of the different sampling frequencies.

\subsection{Data File Format}

Data files were saved as CSV files automatically when the activated logger box started to record. For Type-1 mats, the name of every data file consisted of the 'data', logging box system number which was unique for every box, the recording date and a 6 digit starting time of the file. For Type 2 mats, the panel number of the data file was included in the file title, as sensor 1 , sensor 2 or sensor 3 .

The first row of the data file is the directory of this file plus file name; the second row is date and time; the third row is not in use; the fourth row is column headings which is very important. The first column is time stamp, second column is sample number for Type-1 
and Type-2 mat data files. For Type-1 mat data files, third and fourth columns contain event and pressure respectively, which were not used in this research. Columns 5 through 76 are the sensor identification numbers. For Type-2 mat data files, third and fourth columns are unused event and motion with constant number, 5th column to 28 th column are the sensor identification numbers. From the fifth row to the end of the file are values of timestamp, sample number and pressure value data for every sensor for both Type-1 and Tpey-2 data files. Type- 1 mat's data file records one set of data every 0.05 seconds and Type- 2 mat's data file records one set of data every 0.1 seconds. However, on the data files, the time stamp is not always exact every 0.05 seconds because the SD card cannot write data in it fast enough. Table 1 and 2 are sample data sheets extracted from real data to illustrate the format of Type-1 and Type-2 data files, respectively. Pressing on every sensor separately of a mat then checking the data file you get, which sensor was matched with the column of data can be found. Figure 14 and 15 are the sensor arrays, labelled with corresponding column headings of txl numbers in data files from Type-1 and Type-2 mats, respectively. For example, the third row and third column sensor is labelled number 3, Fig. 14. The data from sensor 3 is recorded in Table 1, under column heading tx13.

The fourth row of a data file contains the sensor number and is essential information for researchers, so they could match sensors with columns represented the sensors value. The TimeStamp column shows the exact time of every sample and the Sample column is the order of samples increasing by one each recording. The sensor's values displayed the applied pressure changing of mats. A threshold of the sensor value was $\operatorname{set}^{1}$, when a cell

\footnotetext{
${ }^{1}$ The threshold was chosen by observing pressure values of the sensors from the bed with no occupants.
} 
value is over the threshold, this sensor is considered loaded and if it is below the threshold, it is considered unloaded.

Table 1: Type-1 mat data file sample

\begin{tabular}{|c|c|c|c|c|c|c|c|c|c|}
\hline \multicolumn{10}{|c|}{ C:IS4Sensorsldata_sn4358_2014_07_17_18_24_35.csv } \\
\hline \multicolumn{10}{|c|}{ Thu Jul 17 18:24:35 2014} \\
\hline TimeStamp & Sample & Event & pressure & txl1 & $\mathrm{tx} 12$ & tx13 & $\ldots$ & tx171 & $\operatorname{tx172}$ \\
\hline 18.24 .35 .390 & 84992 & 0 & 0 & 480 & 508 & 603 & $\ldots$ & 325 & 283 \\
\hline 18.24 .35 .437 & 84993 & 0 & 0 & 500 & 529 & 629 & $\ldots$ & 321 & 281 \\
\hline 18.24 .35 .484 & 84994 & 0 & 0 & 480 & 507 & 602 & $\ldots$ & 323 & 281 \\
\hline 18.24 .35 .531 & 84995 & 0 & 0 & 480 & 507 & 602 & $\ldots$ & 325 & 283 \\
\hline
\end{tabular}




\begin{tabular}{|c|c|c|c|c|c|c|c|c|}
\hline 12 & 8 & 4 & 24 & 20 & 16 & 36 & 32 & 28 \\
\hline 48 & 44 & 40 & 60 & 56 & 52 & 72 & 68 & 64 \\
\hline 11 & 7 & 3 & 23 & 19 & 15 & 35 & 31 & 27 \\
\hline 47 & 43 & 39 & 59 & 55 & 51 & 71 & 67 & 63 \\
\hline 10 & 6 & 2 & 22 & 18 & 14 & 34 & 30 & 26 \\
\hline 46 & 42 & 38 & 58 & 54 & 50 & 70 & 66 & 62 \\
\hline a & 5 & 1 & 21 & 13 & 17 & 33 & 29 & 25 \\
\hline 45 & 41 & 37 & 57 & 53 & 49 & 69 & 65 & 61 \\
\hline
\end{tabular}

Figure 14: Type-1 mat sensor array, numbering system corresponding to txl column, table 1. 
Table 2: Type-2 mat data file sample

\begin{tabular}{|c|c|c|c|c|c|c|c|c|c|}
\hline FILE $=$ /sensor & 1 sn 4426 & 2013 & 7_10_12 & 105 & & & & & \\
\hline Wed Jul 1012 & $: 54: 052$ & & & & & & & & \\
\hline TimeStamp & Sample & Event & Motion & txl1 & tx12 & tx13 & $\ldots$ & tx123 & txl24 \\
\hline 12.54 .06 .439 & 1 & 0 & 1 & 509 & 368 & 528 & $\ldots$ & 399 & 323 \\
\hline 12.54 .06 .539 & 2 & 0 & 1 & 510 & 367 & 528 & $\ldots$ & 400 & 324 \\
\hline 12.54 .06 .628 & 3 & 0 & 1 & 510 & 369 & 529 & $\ldots$ & 399 & 324 \\
\hline 12.54 .06 .866 & 4 & 0 & 1 & 509 & 369 & 528 & $\ldots$ & 400 & 324 \\
\hline
\end{tabular}

\begin{tabular}{|l|l|l|l|l|l|l|l|}
\hline 4 & 16 & 3 & 15 & 2 & 14 & 1 & 13 \\
\hline 8 & 20 & 7 & 19 & 6 & 18 & 5 & 17 \\
\hline 12 & 24 & 11 & 23 & 10 & 22 & 9 & 21 \\
\hline
\end{tabular}

Figure 15: Type-2 mat sensor array, labelled according to respective txl column, table 2. 


\subsection{Data Storage}

Data were stored on SD cards for every logging box or on a computer as the data were first collected. Every month the data was copied and stored onto an encrypted hard drive and then copied again onto a secure cloud server. The encrypted hard drive was password protected and stored in a locked locker in a lab at Carleton University. Only researchers involved in the project who had signed a pledge of confidentiality had permission and a password to be able to access data on the server. 


\section{Chapter 4: Center of Pressure Trajectory}

\subsection{Introduction}

This chapter describes Center of Pressure (COP) location ${ }^{2}$. Various techniques have been described for analyzing COP information relevant to postural control of standing and walking [60], [63]-[65]. Poor postural control is associated with a higher risk of falling, which can lead to hospitalization, particularly among older adults [66]. Since postural control is also important for being able to rise independently [67], it follows that the development of techniques for analyzing COP related to getting out of bed are important to the development of home monitoring systems.

COP analysis approaches in the past have been restricted to examining movement direction [43] rather than considering movement direction in relation to time, frequency and magnitude of movement patterns. This chapter describes an advanced approach for analyzing COP information relevant to bed mobility which incorporates information on movement direction as well as time, frequency and magnitude of movement patterns. The key contribution is that it provides a method for monitoring and analyzing postural control data which has relevance to an older population at risk of falling. Sway is one

\footnotetext{
2 The use of the term "center of pressure" may vary according to the field it is used in. According to Meriam Webster dictionary, it is defined as "the point of a surface exposed to external pressure (as of a fluid) at which a single force must be applied to equal or counterbalance the pressure forces acting on the whole surface". The definition used in this thesis work is given in sub-section 4.2.1 of this thesis.
} 
aspect of COP trajectory, related to fall risk. Increased postural sway in standing has been associated with balance performance [68]. The technology and algorithms in this thesis are aimed at determining sway that older people exhibit while sitting on and exiting their beds. This will allow researchers a way of monitoring postural control in the home environment during an important function that is performed daily by most people.

In particular, the thesis looks at a way of capturing the movement trajectories that occur in both the $\mathrm{x}$ and $\mathrm{y}$ directions (i.e. bed length and bed width) and provides a way of distinguishing the amount of sway that occurs in either direction.

\subsection{Algorithm}

This section discusses how the algorithm was used for calculating COP trajectory in the $\mathrm{X}$ and $\mathrm{Y}$ directions over time as well as the frequency and extent of participant movements during bed exits and on the bed.

\subsubsection{COP 3-D trajectory}

An algorithm was written in MATLAB to process the CSV files collected and to analyze information relevant to COP. Data were sampled at a frequency of $10 \mathrm{~Hz}$ with Type-2 mats and at $20 \mathrm{~Hz}$ with Type-1 mats. A threshold level was set for each sensor to determine whether it was loaded or not (i.e. whether an individual was in the bed). The reason we use a threshold is that sensors detected pressure exerted by the overlying 
mattress and therefore produced a pressure value even when participants were absent from bed. These unloaded pressure values were not taken into account by the algorithm which considered sensors to be loaded when producing pressure values above the set threshold.

As mentioned previously, coordinate $\mathrm{X}$ corresponded to bed width while coordinate $\mathrm{Y}$ corresponded with bed length. The pressure sensitive mat had 8 sensors at every line in the $\mathrm{X}$ direction and 9 sensors at every line in the $\mathrm{Y}$ direction. The algorithm designated each column in the $\mathrm{X}$ direction with an index from 1 to 8 and each row in the $\mathrm{Y}$ direction with an index from 1 to 9 . The $\mathrm{X}$ coordinate of the $\mathrm{COP}$ was calculated as weighted sum of the $\mathrm{X}$ coordinates of the loaded sensors. The weighting coefficients are the corresponding sensors pressure values. The $\mathrm{Y}$ coordinate of the COP was computed as the weighted sum of the $\mathrm{Y}$ coordinates of the same set of sensors used for computing the $\mathrm{X}$ coordinate. In both cases, the weighted sum was divided by the sum of pressure of the loaded sensors. Combining results from both sets of coordinates produced a COP location on the mat area. Using this information a three dimensional (3-D) graph showing (X, Y) location trajectory over time which corresponded to a sample taken every 0.05 seconds or 0.1 seconds could be plotted. Data were also used to create a movement frequency and magnitude plots, such as shown in Fig. 25. This showed the temporal variations of movements in the $\mathrm{Y}$ direction by using different colors and the frequency of movements changes.

'Gold standard' is the best available way to do test under reasonable conditions [69]. Observing a subject's movements on the bed and recording all the movements can be treated as a 'gold standard'. In this thesis, we used this 'gold standard' test to verify COP 
trajectory algorithm using the lab set-up system on a young female, meanwhile video was taken. The tests as follows:

1. The subject sat on the lab's bed, leaned to her left side then leaned to her right side as shown in Fig. 16 and Fig. 17 (from the video). The corresponding COP trajectory is shown in Fig. 18 and Fig. 19. Figure 18 shows the $\mathrm{X}$ and $\mathrm{Y}$ plots of a 3-D plot, corresponding to the subject movements on the $\mathrm{X}$ direction and $\mathrm{Y}$ direction separately. In Fig. 18, the region T1 corresponds to the subject's moving close to the foot of the bed (Y direction). A large change is observed on the COP in the $\mathrm{Y}$ direction whereas the $\mathrm{COP}$ on the $\mathrm{X}$ direction had a small change in the region between samples 220 and 300 . T2 points at the area where COP on the $\mathrm{Y}$ direction moves far from the foot of the bed and the COP on X direction did not have much change from samples 350 to 450 . Figure 19 is a 3-D COP movements' trajectory plot from this test.

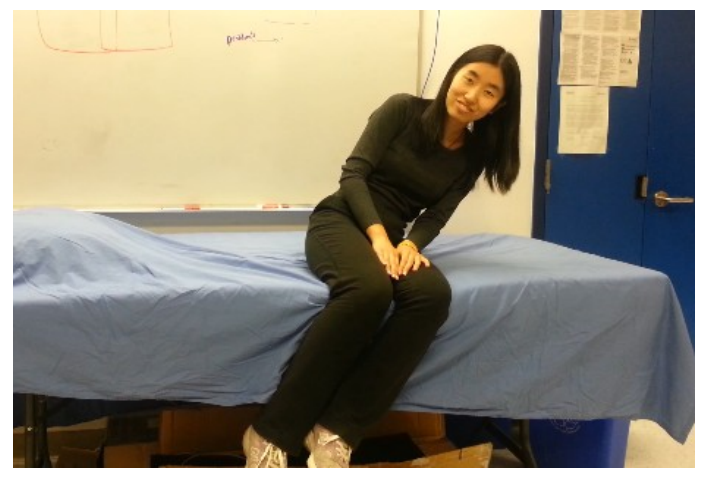

Figure 16: The subject leans to left.

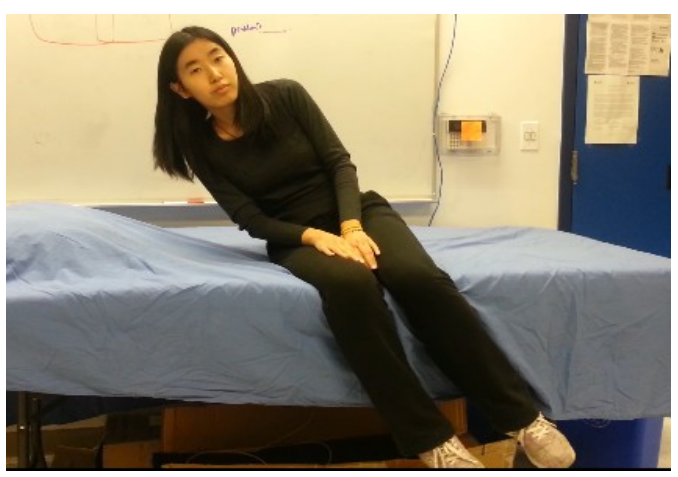

Figure 17: The subject leans to right. 

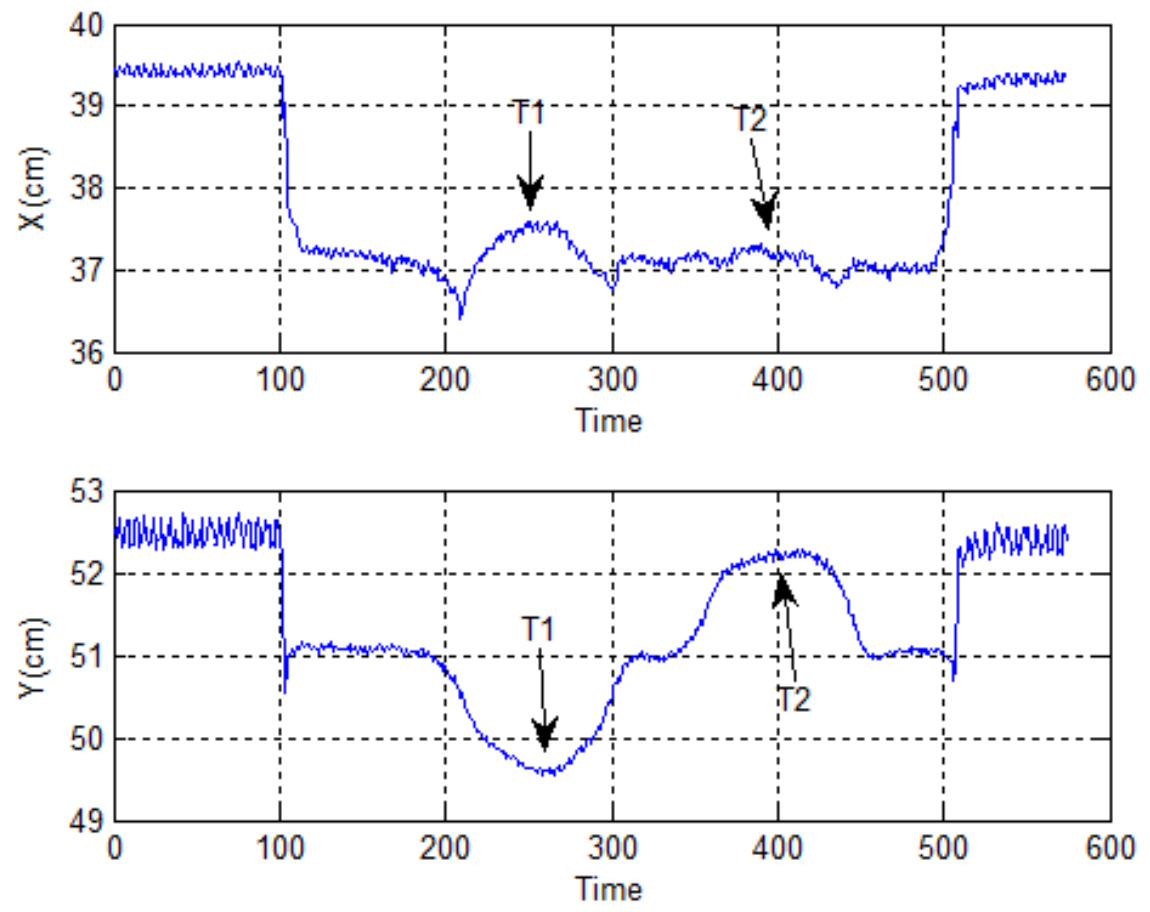

Figure 18: time-X and time-Y trajectory plots versus time (in sample intervals) of lab test 1.

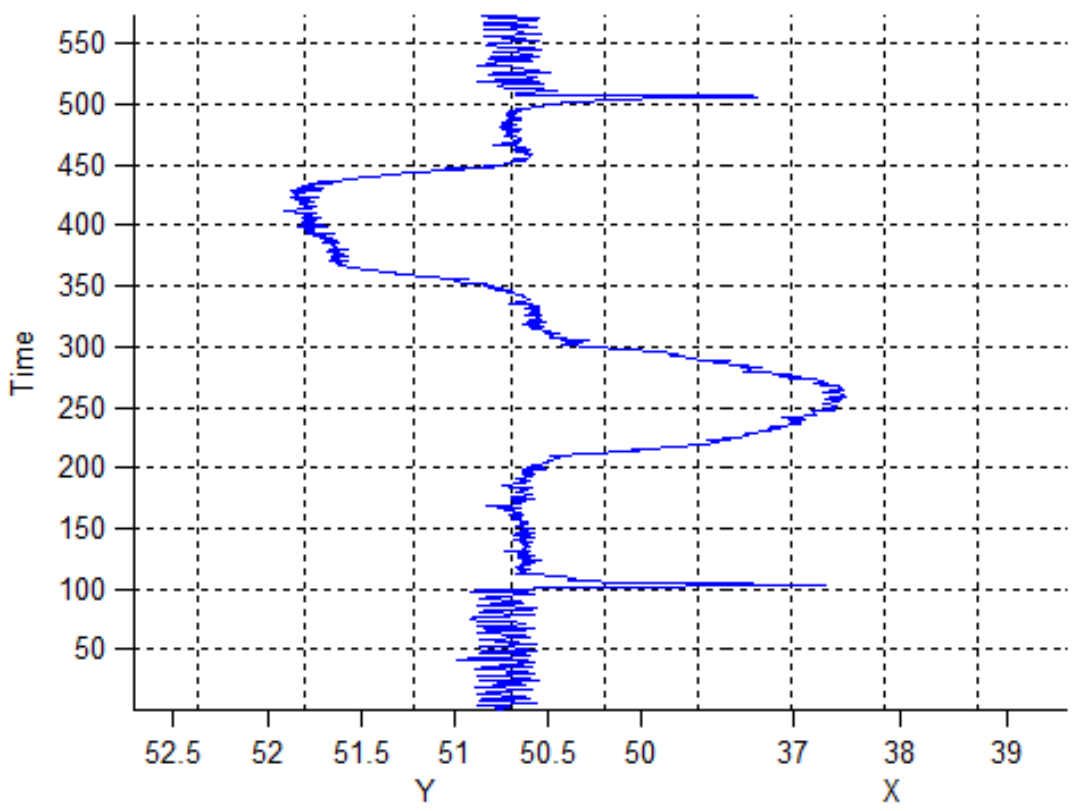

Figure 19: The COP 3-D trajectory plots of test 1 (used "Rotate 3D" function to make the plot readable). 
2. The subject sat straight, and then leaned backward as shown in Fig. 20 and 21.

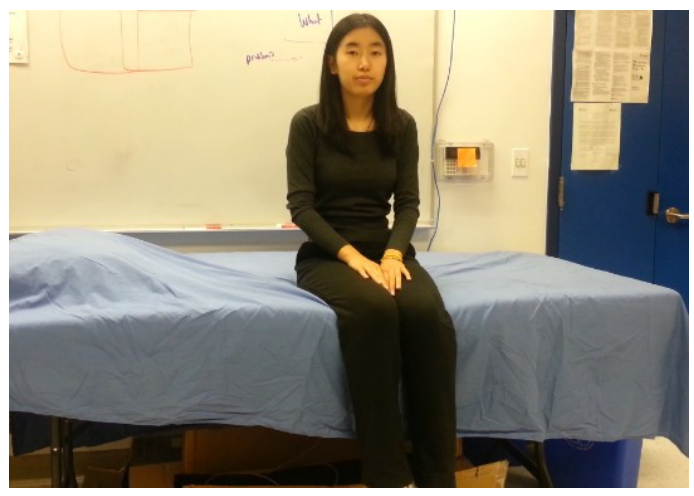

Figure 20: The subject sits straight.

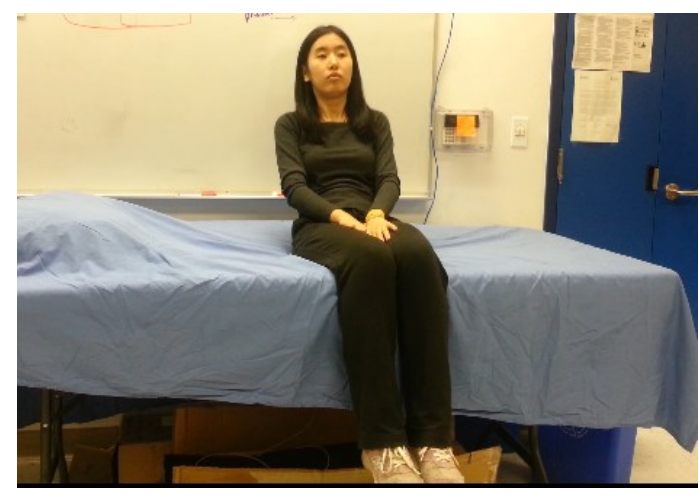

Figure 21: The subject leans backward.

The COP trajectory is shown in Fig. 22. T1 points to the area where the subject's COP is stable, and T2 points to the area where the subject leaned backward.
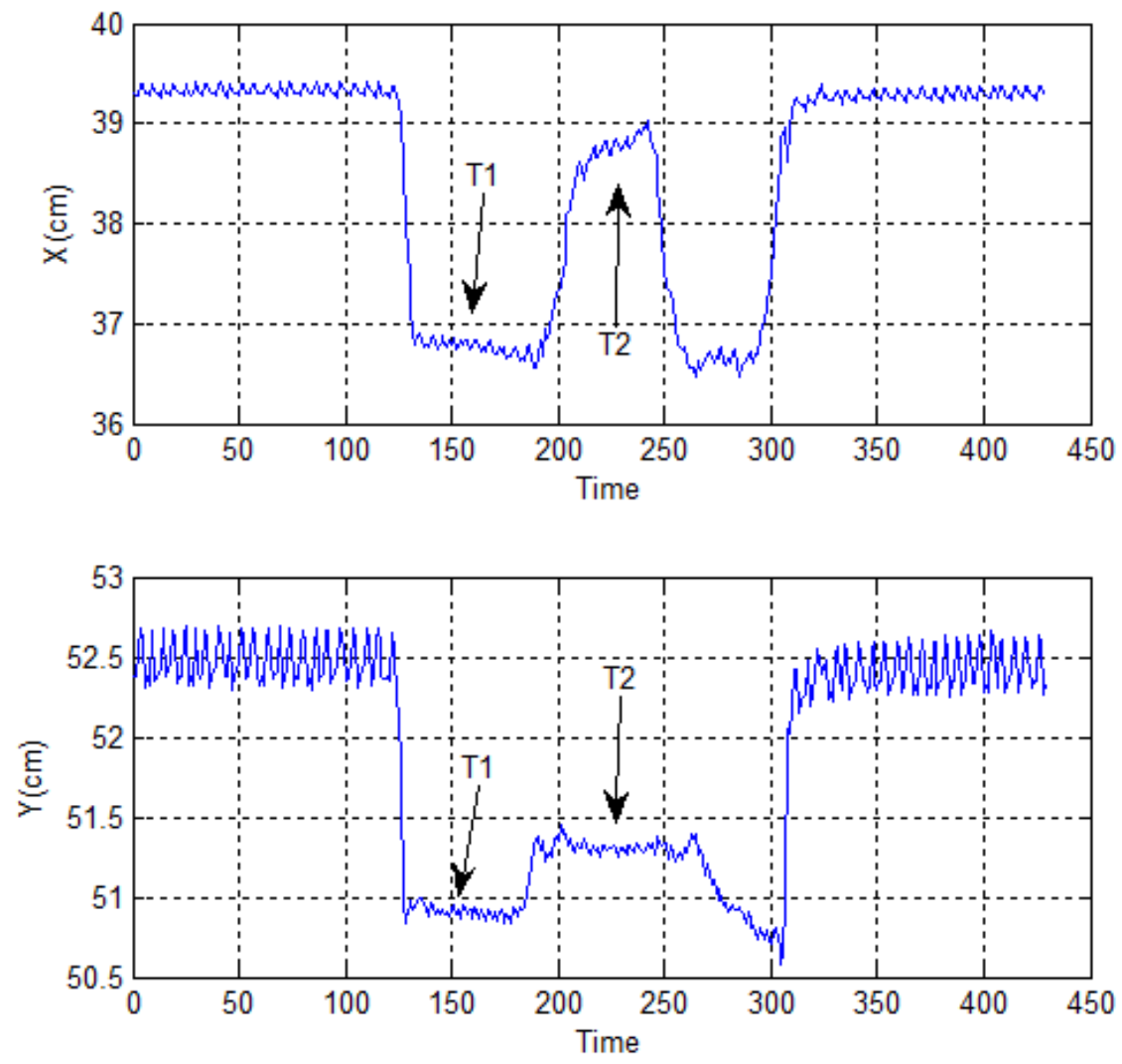

Figure 22: time-X and time- $Y$ trajectory plots versus time (in sample intervals) of lab test 2 . 
Figure 23 shows the 3-D COP trajectory plot of this test.

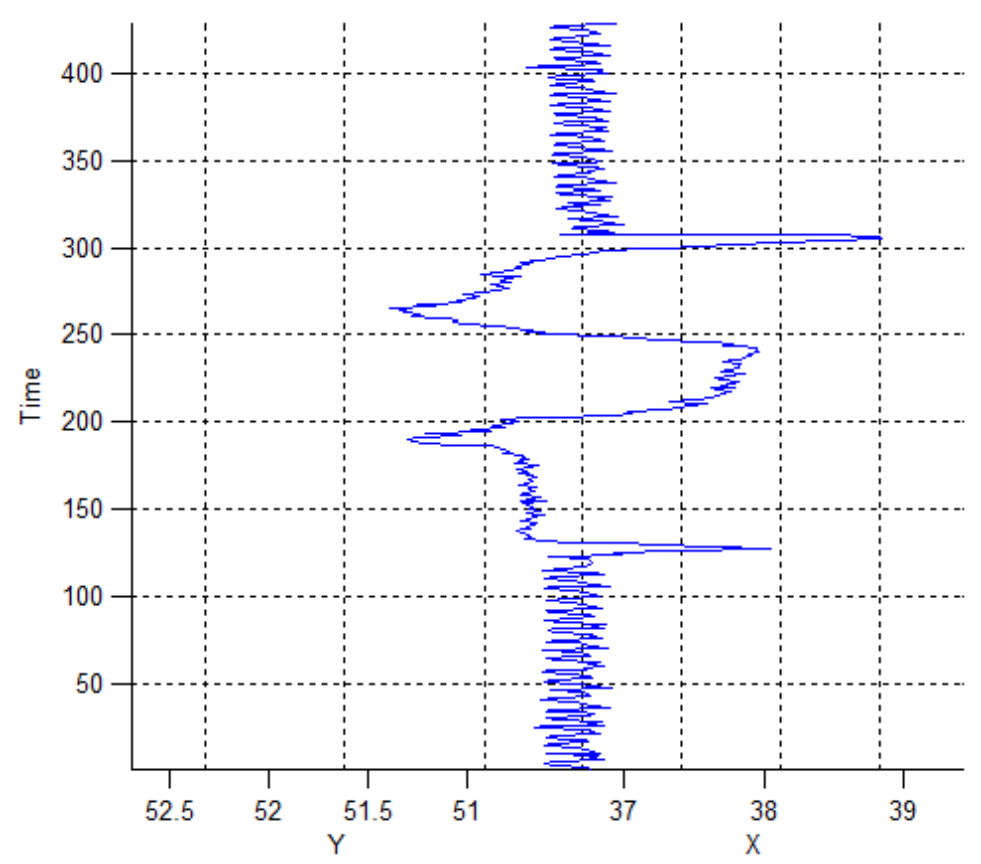

Figure 23: COP 3-D trajectory plots from test 2 (used "Rotate 3D" function to make the plot readable).

Figures 18 and 19 verified the algorithm of COP in terms of X direction (COP-X) trajectory movements. When the subject leaned to the left and right, COP did not change much at $\mathrm{X}$ axis which was the short side of the bed. It changed at $\mathrm{Y}$ axis, the long side of the bed. It can be seen from Fig. 19, that at $\mathrm{Y}$ axis there were clearly two big movements at two opposite directions which indicated the subject leaned to the left and right. The small bumps at $\mathrm{X}$ axis shown at Fig. 18 were able to be matched with the video. They were the volunteer slightly leaning backward so the $\mathrm{COP}$ at $\mathrm{X}$ direction on the plot has fluctuations. Figure 22 and 23 verified the algorithm of COP in terms of Y direction (COP-Y) trajectory movements. T1 was matched with the video that the person sat still, 
COP did not move in both $\mathrm{X}$ and $\mathrm{Y}$ directions. When the person leaned backward, the main COP change was at $\mathrm{X}$ direction, and $\mathrm{Y}$ direction did not have a big movement as Fig. 22 shown. However, From Fig. 22 we can see Y direction also had a small hill at T2, that was because when a person moves it is impossible for her to move $100 \%$ straight.

Figure 24 was an example of a COP trajectory plot generated with data collected from a young healthy female as she performed sequential various tasks related to bed mobility. From this plot, one can see there are 5 distinct stages of movement labeled S1 to S5. The $\mathrm{x}$ axis was time which used samples to represent, and $\mathrm{y}$ axis and $\mathrm{z}$ axis were locations which displayed in millimeter. These points and the paths represent the following sequence of movements: $\mathrm{S} 1$ = sitting on edge of bed; $\mathrm{S} 1$ to $\mathrm{S} 2$ path = lying down; $\mathrm{S} 2=$ supine lying in stable position; S2 to S3 path = rolling to side of bed where previously sitting; S3 = stable side lying position; $\mathrm{S} 4$ = sitting on edge of bed but closer to foot of bed than S1 starting position; S4 to S5 path = getting out of bed, S5 = out of bed. One can see from the graph that the largest movement on the $\mathrm{Y}$ axis was lying down whereas rolling and going from sit to stand occur mainly in the X-direction.

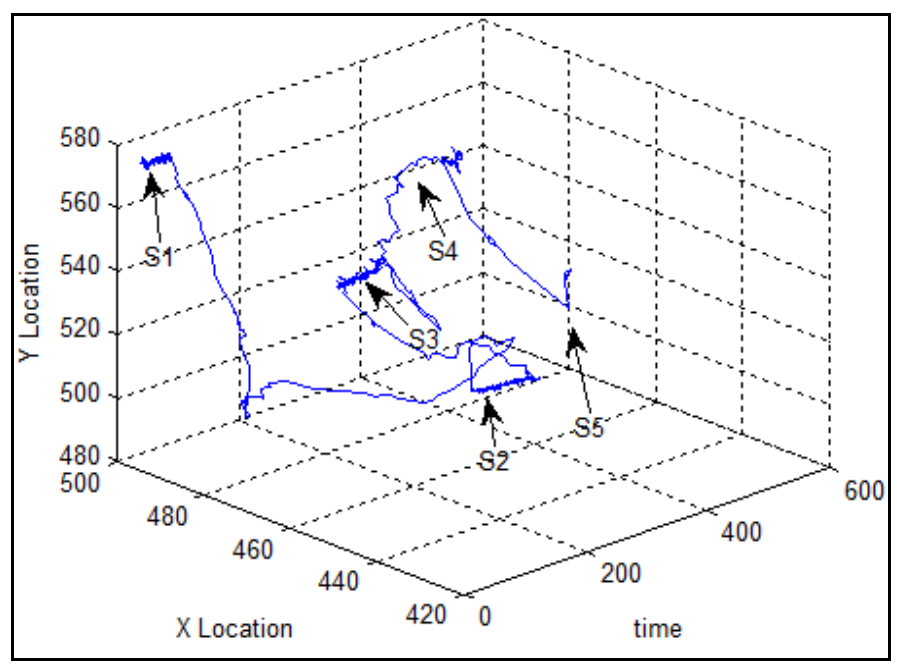

Figure 24: Example of COP plot (time in sample intervals). 
The temporal variations of the COP Y-coordinate are depicted in Fig. 25. This figure was associated with the same movement sequence as performed by the young participant and shown in Fig. 24. In Fig. 25, the horizontal axis represents the time in sample numbers. The color difference represents the subject's distance in Y direction of the COP. The color changes from blue color to red color represents the distance changes from 500 millimeters to 570 millimeters. From Fig. 25, one can see in the first 60 time samples that the participant produced a large $\mathrm{Y}$ value represented by dark red followed by smaller $\mathrm{Y}$ value represented by yellow. The color change from dark red to yellow indicates there was a large magnitude COP movement in the $\mathrm{Y}$ direction for the given time period which corresponded to her position change from sitting to supine lying. Similar graphs could be generated for COP X-coordinate.

The rest of the sequence of movements had a smaller magnitude than the initial change from S1 to S2 corresponding to Fig. 24. From S2 to S3 (i.e. supine lying to side lying) there was a comparably smaller magnitude movement in the $\mathrm{Y}$ direction which is represented by the change from light blue to dark blue and back to light blue around the 250 time mark. In essence, this plot uses color contrasts ranging from dark blue to dark red to represent whether a movement in the $\mathrm{Y}$ direction was large or small in magnitude at any given time in the movement sequence. The width of a color band within a time coordinate time shows how long the participant stays in a posture. The key of visualizing the color contrast plot is horizontal color changes. It shows how fast the movement changes in $\mathrm{Y}$ direction. 


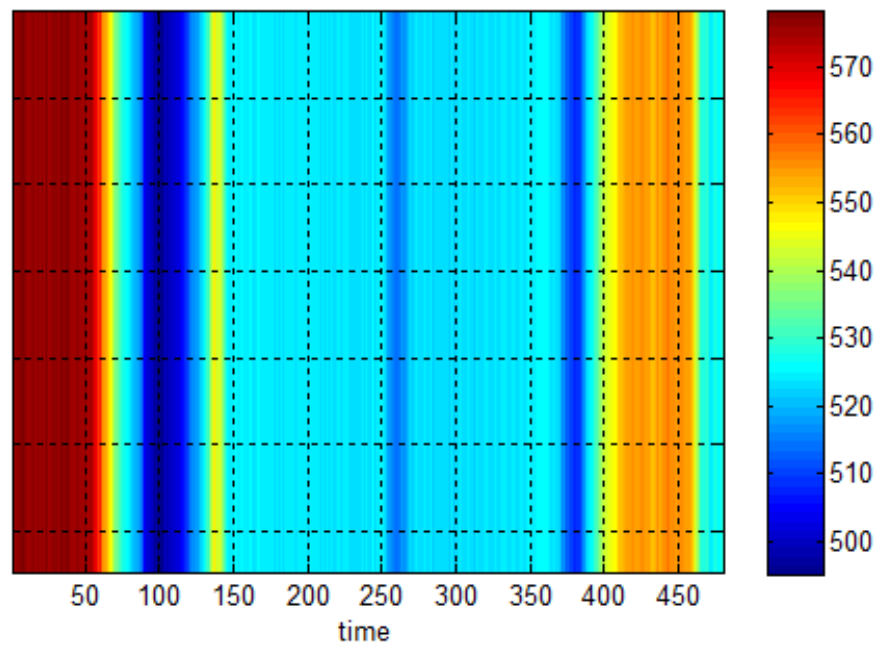

Figure 25: Color contrast plot showing the temporal variations of the Y-coordinate of the COP.

\subsubsection{Time-X trajectory}

Each 3-D plot was converted into two 2-D plots to get a clear image of movement in different directions to help on analyzing 3-D COP trajectory plots. The 2-D plots have been used to verify the algorithms in section 4.2.1. Time-X plots, is $\mathrm{x}$ axis along with time, $\mathrm{y}$ axis along with the $\mathrm{x}$ direction of the mat which is the width side of the bed. From time-X trajectory plot, movement along the width of the bed can be displayed. In other words the time-X trajectory plot shows how a person's COP changes as he turns from one side to the other side of a bed or how COP changes as a person moves out of a bed. Figure 26 is a break-down of the plot from Fig. 24 in the X direction only. 


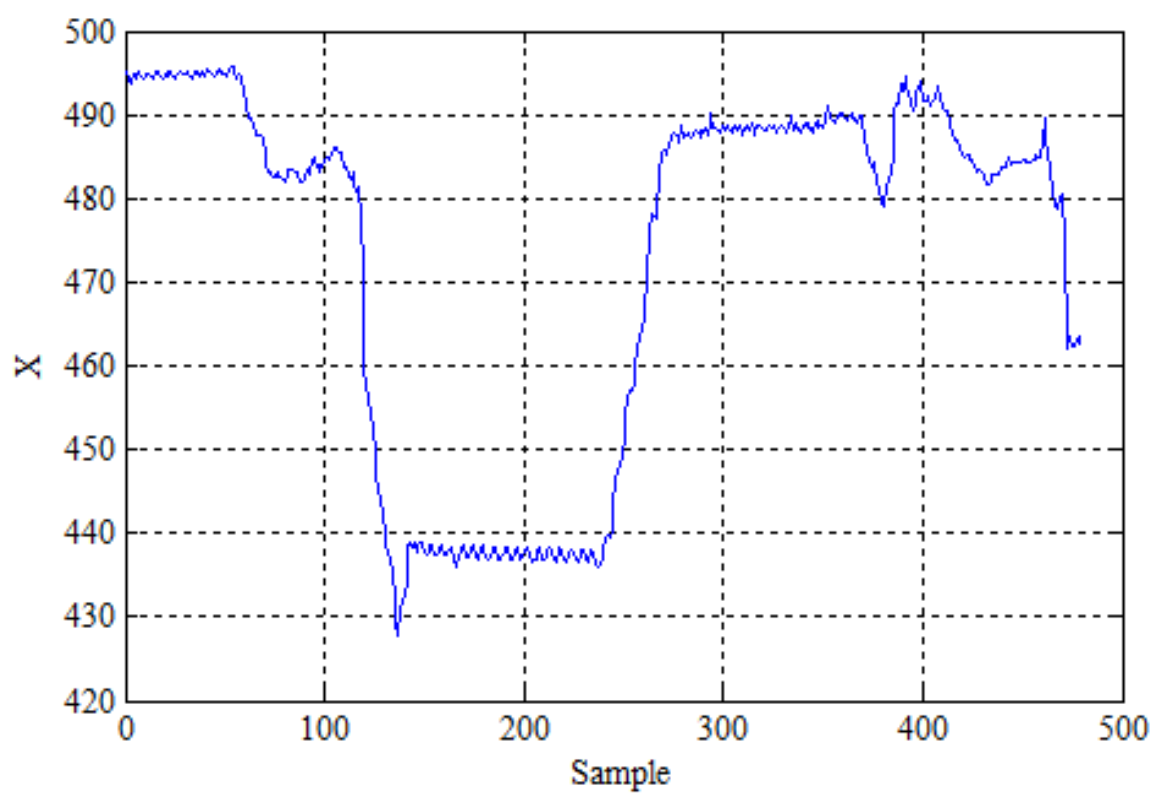

Figure 26: $\mathrm{X}$ direction movement with sample sequence.

The time axis of Fig. 26 used sample index, and y axis used the sensor row number multiplied by 100 to make movements more clear. Figure 27 shows the time axis as the real time of the data and y axis shows the location in centimetre, from $0 \mathrm{~cm}$ to $80 \mathrm{~cm}$.

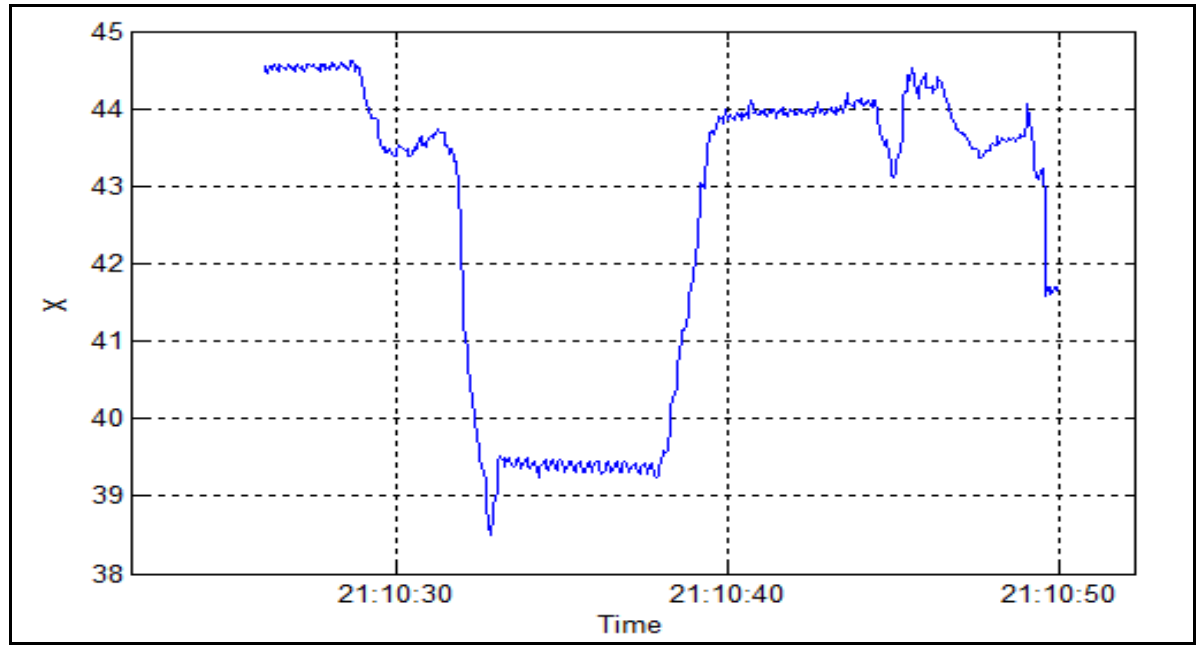

Figure 27: Time-X plot with real time and $\mathrm{cm}$ as unit for $\mathrm{y}$ axis. 


\subsubsection{Time-Y trajectory}

If the time-X trajectory was the only aspect of COP examined, information on movement occurring along the bed length would be missing. When a person changes position from lying to sitting, a large amount of sensors' pressure change occurs along the bed length. Therefore obtaining a time-Y trajectory plot is necessary for a more complete picture. Time- $\mathrm{Y}$ plot has sample index as $\mathrm{x}$ axis, and $\mathrm{Y}$ direction of the mat as $\mathrm{y}$ axis. Figure 28 is the break down plot of $\mathrm{Y}$ direction COP from Fig. 14. The plot gave us additional information about how a person performed a bed-exit and how he moved before he got out of a bed. For instance, as a subject's position changes from sitting to lying, there is a corresponding trough in the COP pressure tracing in the $\mathrm{Y}$ direction of the mat. This trough can be seen on the beginning of time-Y trajectory plot in Fig. 28. The ability to detect this aspect of movement may be useful to detect bed exit symmetry changes that can occur with age, injury or illness. Theoretically, poor symmetric problem caused movements during bed-exit could be captured in the COP changes on the time-Y plot if they occurred along the bed length. Conversely if rocking action occurred along bed width it can be captured by time-X plot. 


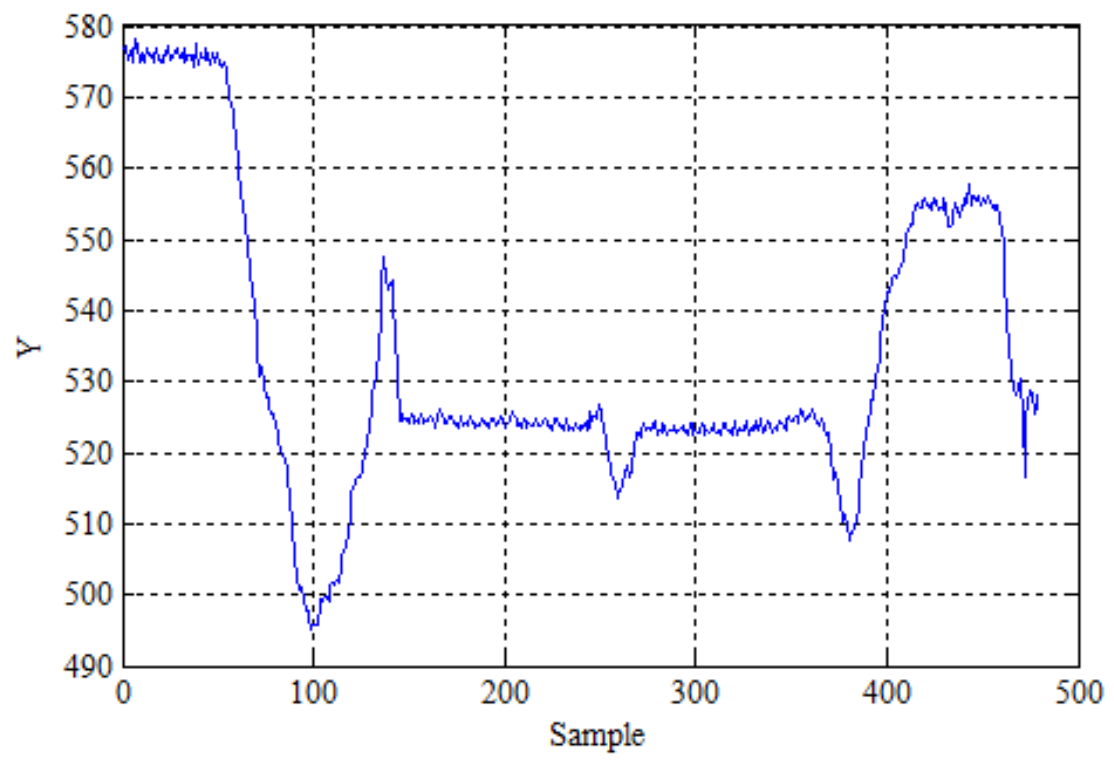

Figure 28: Y direction movement with sample sequence.

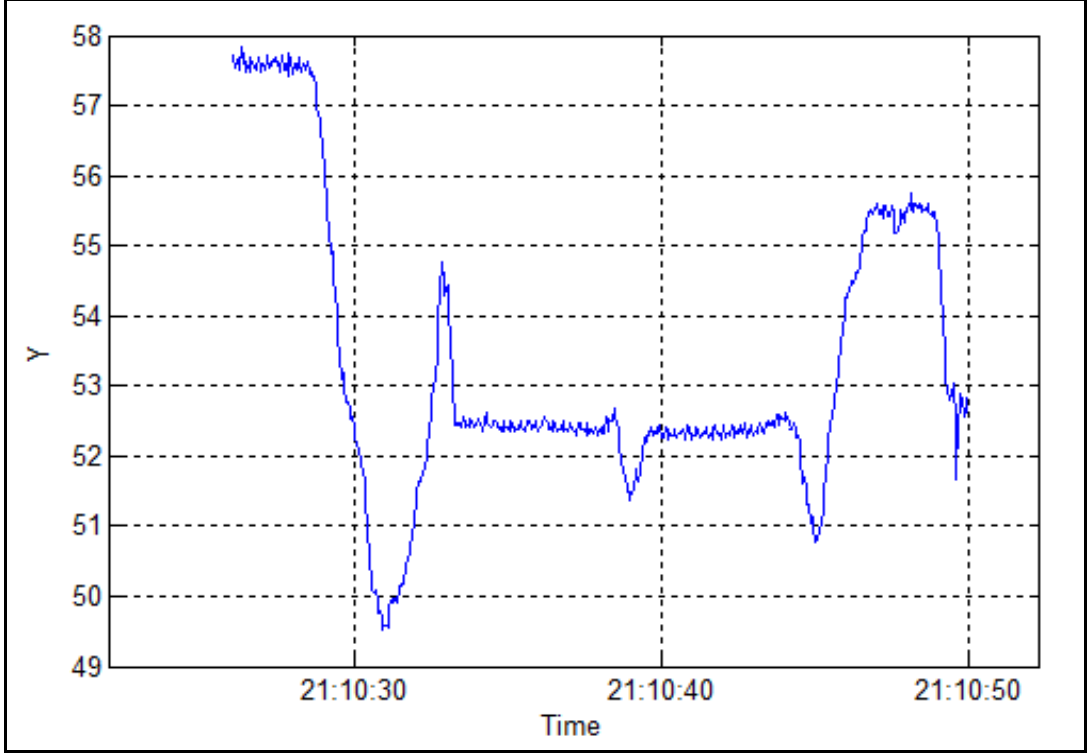

Figure 29: Time-Y plot with real time and $\mathrm{cm}$ as unit for $\mathrm{y}$ axis.

Figure 29 the time axis was the real time of the movements happened; y axis was the Y direction of centimeter in the coverage area of mats from $0 \mathrm{~cm}$ to $90 \mathrm{~cm}$. 


\subsection{Feature Differences}

\subsubsection{COP trajectory in 3-D graph difference before and after a fall}

The algorithm described in section 4.2 , has been used to analyze COP bed exit data collected from an older participant in relation to a fall she experienced. The data were provided to us by our clinical partners in a full anonymized way. The participant recorded the date of this fall on a calendar collected by the researcher. The COP pattern was analyzed for all bed exits which occurred a week before and a week after the participant fell (approximately 40 exits in total). Figure 30 illustrates a typical pattern of her first morning bed exit. This particular transfer was chosen to represent her typical pattern since the timing corresponded to her average monthly bed exit sit-to-stand time as measured by a previously developed algorithm [33]. The time period sampled in Fig. 30 was about 1 hour to include a period of quiet lying prior to the bed exit. 


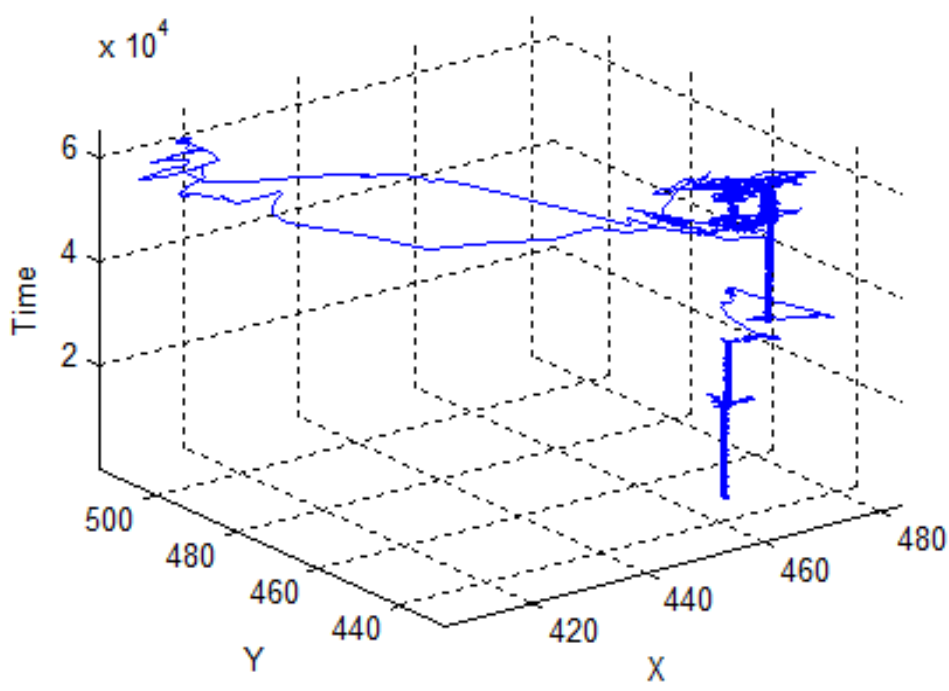

Figure 30: Morning exit of four days before the fall (time axis shows as sample sequence).

In Fig. 30, the COP path indicates that she normally rolled to one side from supine lying and then got into a stable position from the straight long perpendicular line to an another period of perpendicular line. The trace shows that the subject leaned to one side while exiting the bed (the big movement). 


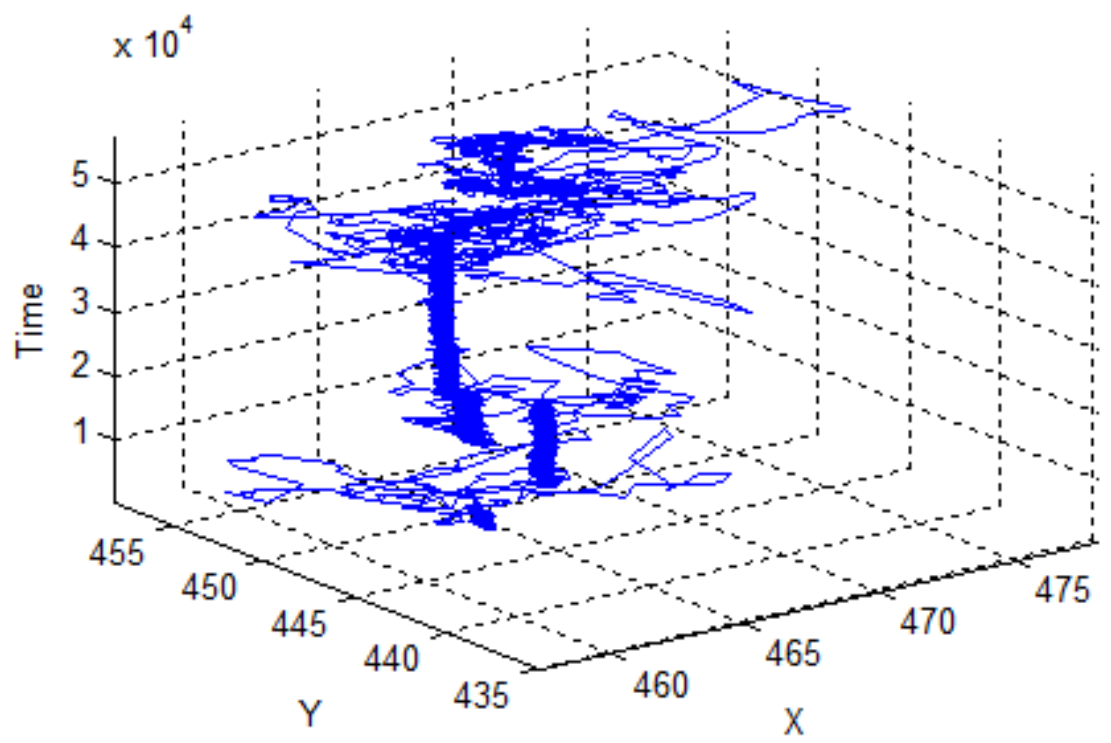

Figure 31: The next morning exit after the fall (time axis shows as sample sequence).

Figure 31 illustrates COP bed exit pattern the first morning after the participant fell. The path in Fig. 31 does not display a long period of quiet lying or a big movement pattern as did the previous plot. Furthermore there is a much larger area of COP movements in both $\mathrm{X}$ and $\mathrm{Y}$ directions prior to her bed exit.

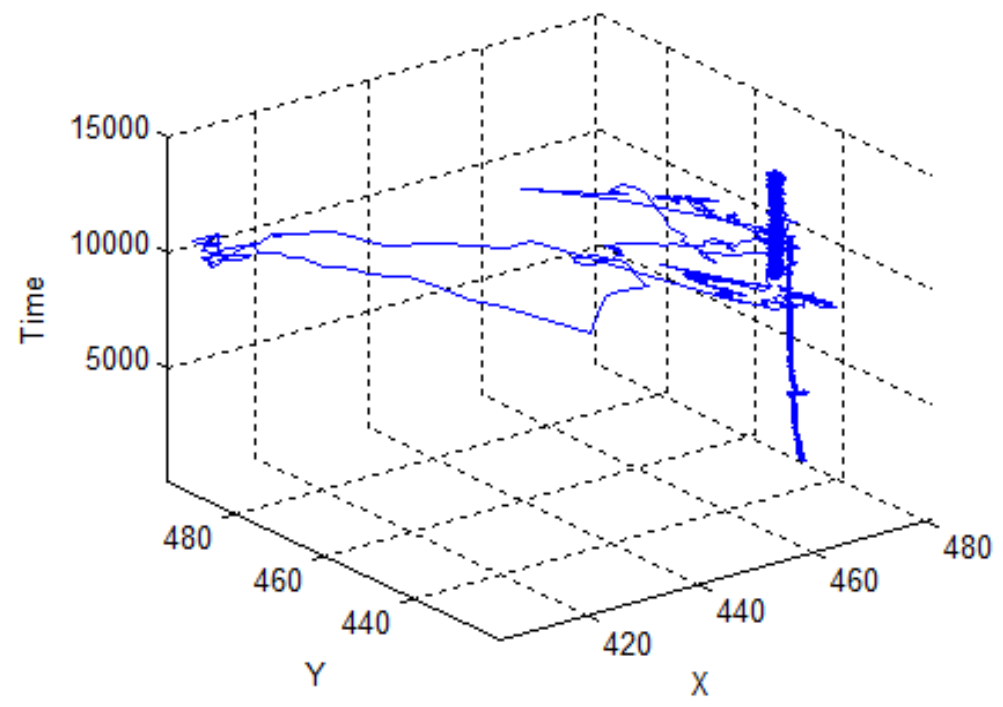

Figure 32: Morning exit four days after the fall (time axis shows as sample sequence). 
Figure 32 illustrates COP movements 4 days after the participant's fall. The COP path trajectory is much closer to the participant's typical pattern based on visualizing. They both have a period of stability prior to the roll into side lying. Before her bed exit, there is also less COP movement indicating greater stability. This pattern is much closer to the typical pattern in Fig. 30 and Fig. 30-32 suggesting that the participant altered the way she got out of bed after falling but returned to her regular pattern 4 days later.

Here $\mathrm{X}, \mathrm{Y}$ axis interval values are slightly different among plots since the sizes of COP movements are different. The MATLAB 'auto tight' function was used to adjust X, Y interval values for each set of data in order to optimize plot clarity.

\subsubsection{Frequency color graph difference}

The color contrasts in Fig. 33 illustrate the magnitude changes of COP movements in the Y direction with time by the color from dark blue to dark red as the color legend show on the side, according to Fig. 30. The narrow band of red shows where the furthest movement in the $\mathrm{Y}$ direction occurred during the sequence. The relatively high degree of color contrast horizontally in Fig. 34 throughout the sampled time period indicates that more movements occurred throughout this bed exit over a longer period of time and the movements' changes were more than Fig. 33. Figure 34 corresponds with Fig. 31 and Fig. 35 corresponds with Fig. 32 . The Fig. 35 color plot shows that initially there was no movement as indicated by the large area of solid light blue color. Following this, there is a small band of high color contrast including yellow, green and red. This high contrast color band indicates higher magnitude movements were performed in a relatively short period of time. 


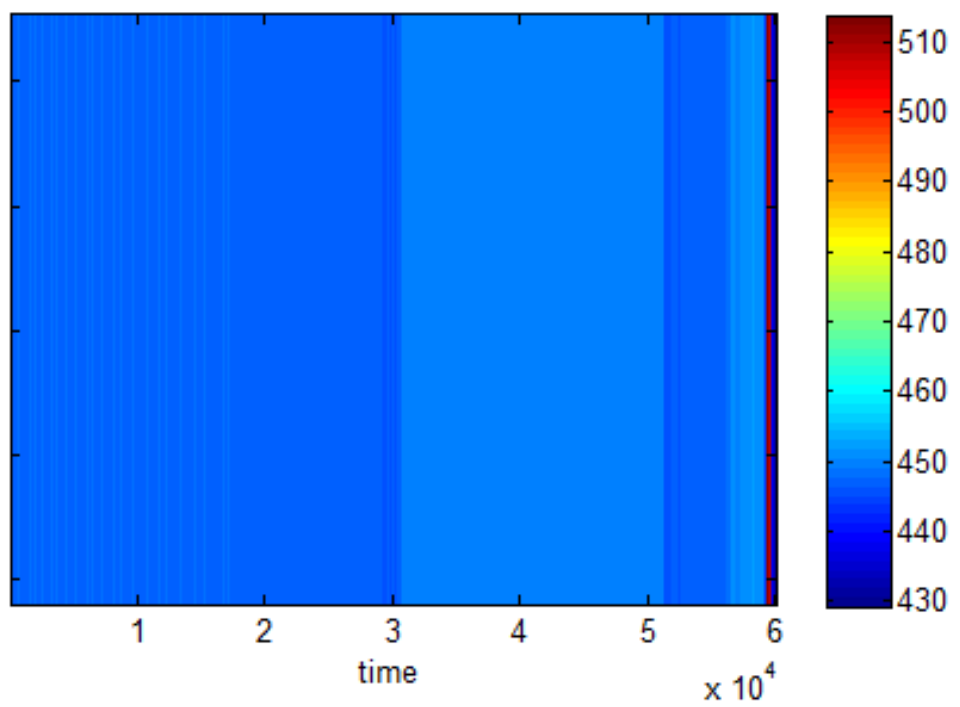

Figure 33: Color contrast plot of the morning exit four days before the fall showing the temporal variations of the Y-coordinate of the COP.

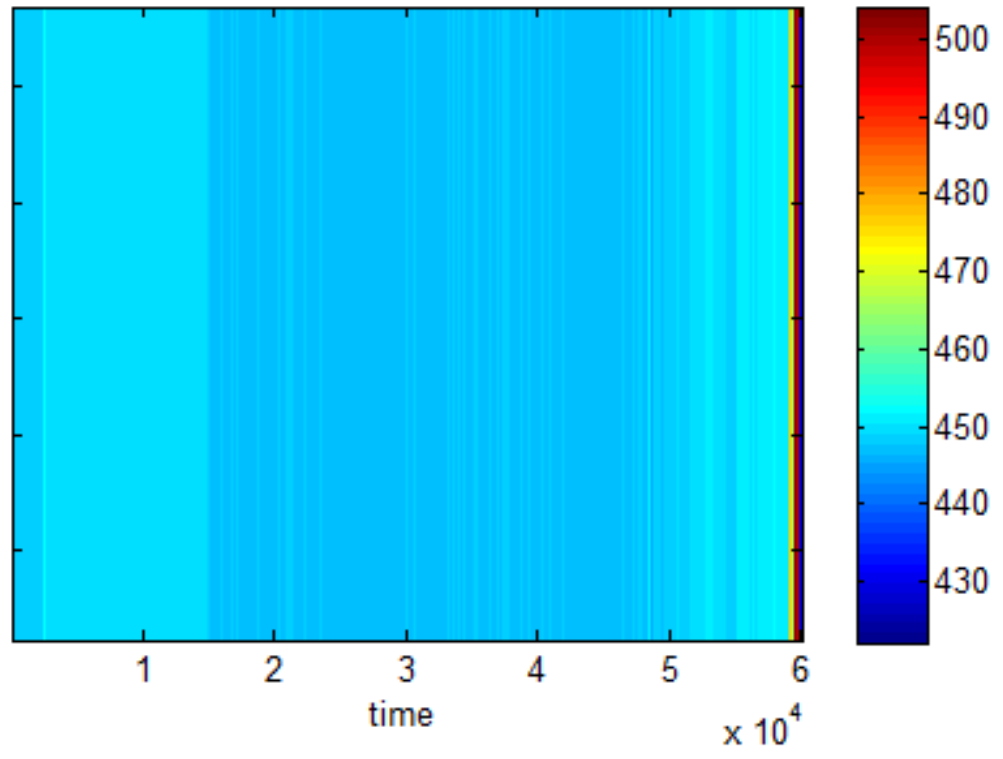

Figure 34: Color contrast plot of the next morning bed exit after the fall showing the temporal variations of the Y-coordinate of the COP. 


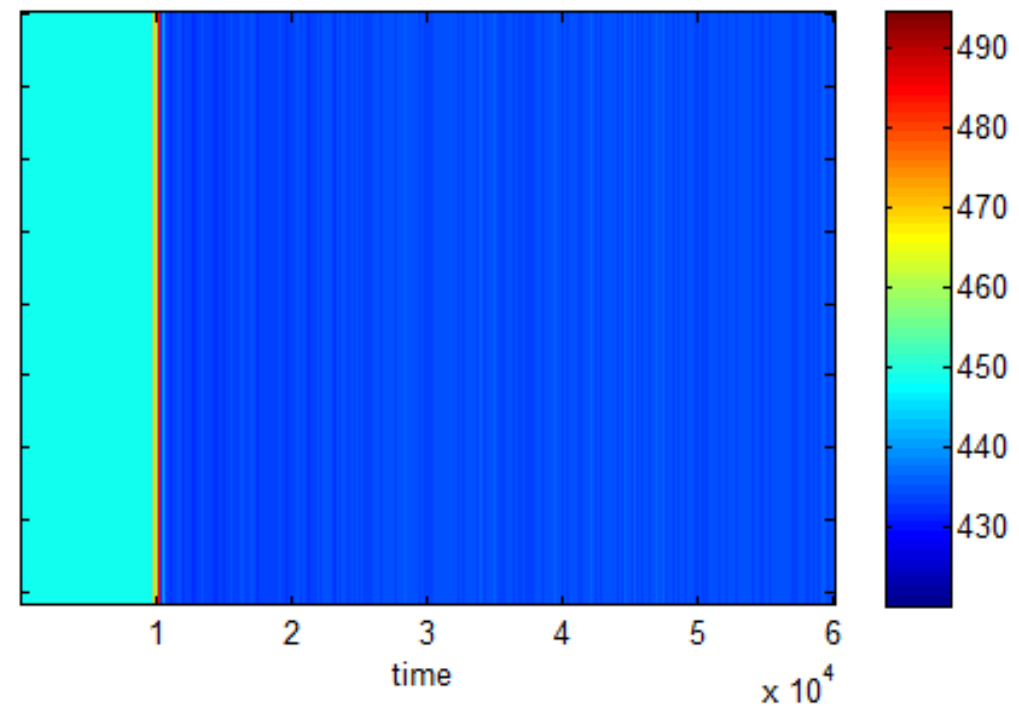

Figure 35: Color contrast plot of the morning exit four days after the fall showing the temporal variations of the $\mathrm{Y}$-coordinate of the $\mathrm{COP}$.

\subsection{Results}

Figures 30 and 33 show a typical way of getting out of bed. From Fig. 31 and 34 we can see there are a large amount of small movements and color contrasts. These differences may have been due to greater pain, instability or restlessness following the fall. After 4 days, this participant's bed-exit pattern is backing to normal, suggesting her health situation and mobility is recovering.

This algorithm of calculating and generating COP trajectory over time introduced in this chapter provides a novel approach for analyzing COP information that has relevance for monitoring bed mobility over time. Using this approach, visual displays of COP data were produced which incorporated movement in $\mathrm{X}$ and $\mathrm{Y}$ directions over time thereby producing a movement trajectory that can be interpreted as a sequence. Furthermore visual color contrast displays were produced that convey additional information regarding 
the magnitude and frequency of COP movement. When tested on data collected from an older participant who experienced a fall, this method of analyzing COP was able to illustrate distinct differences in bed exit patterns used pre and post fall episode. The approach, therefore shows promise in terms of being able to detect critical mobility changes and is worthy of ongoing development.

For comparison with older adults bed exit patterns, the algorithms were also tested on young people who are at their 20s. Five young people performed the task of laying stable and then getting out of bed in their normal way, including LTS and STS periods. Figure 36 and 37 represent one young volunteer's same bed-exit transfer from two directions. Time axis of this two plots shows that this young volunteer took a very short time getting out of the bed. Her movements were smooth and there were no redundant movements. Figure 38 is 3-D COP trajectory of the same transfer with Fig. 36 and 37. In the first three seconds the young volunteer was lying on the bed without moving so the trajectory line is straight. Following the initial period of stability she started to roll to one side while sitting up and then stood up without a pause. After she got out of the bed, the trajectory line returned to the middle of bed meaning no one was on the bed.

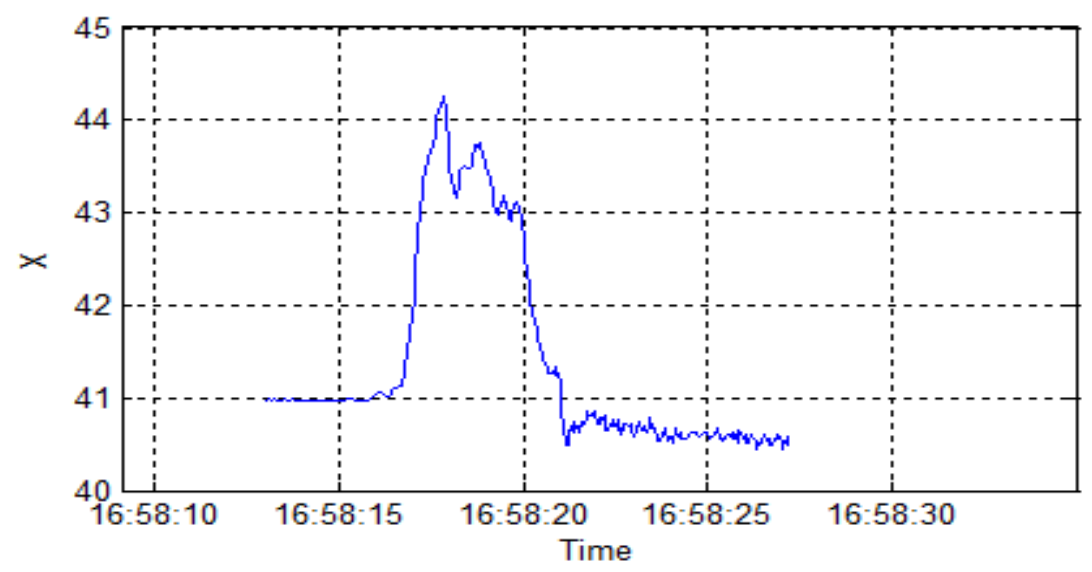

Figure 36: A young person getting out of the bed $\mathrm{X}$ direction plot. 


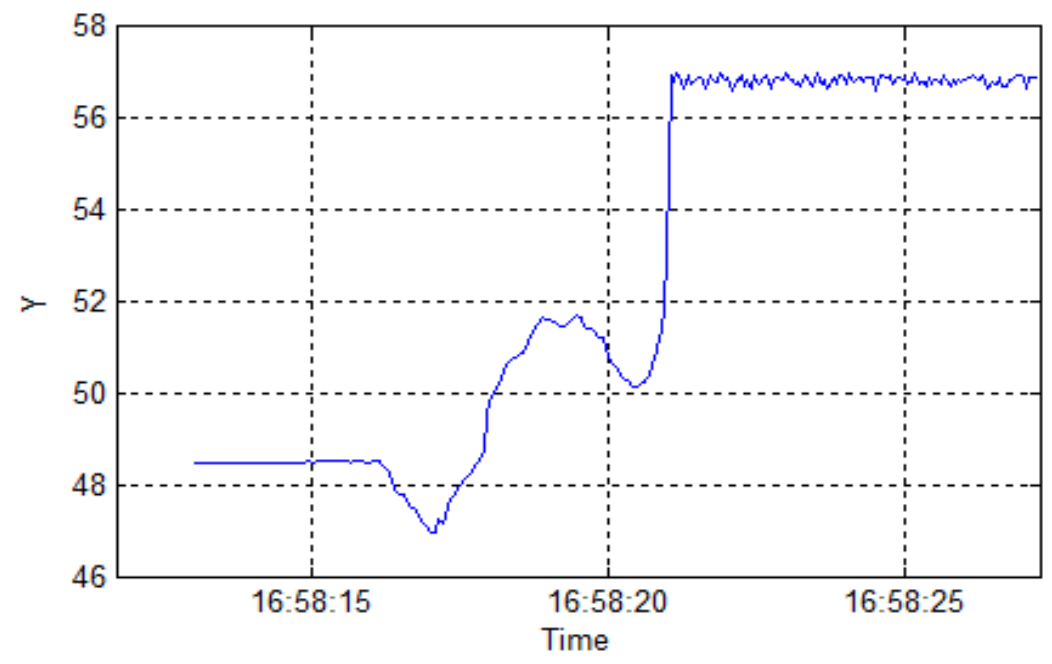

Figure 37: A young person getting out of the bed $\mathrm{Y}$ direction plot.

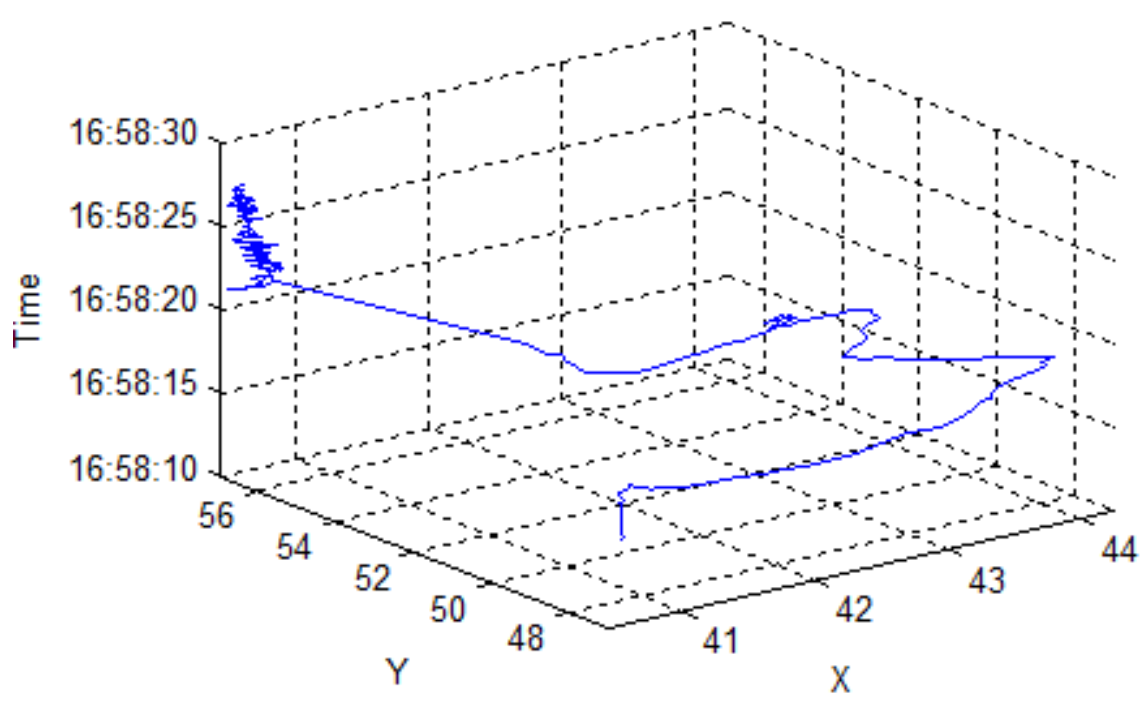

Figure 38: This young person bed-exit COP trajectory plot.

Below Fig. 39 shows three sets of plots from three young volunteers. They all got out of the bed in their own habitual ways. The first two individuals combined from lying to sitting and moving to one side of the bed two motions, had a small pause before getting out of bed. The third young person combined all three motions in one. From young 
people's COP trajectory plots and break-down time-X and time-Y plots, all showed that young people have similar bed exit patterns on bed-exit transits which are more smooth and faster than seniors'.
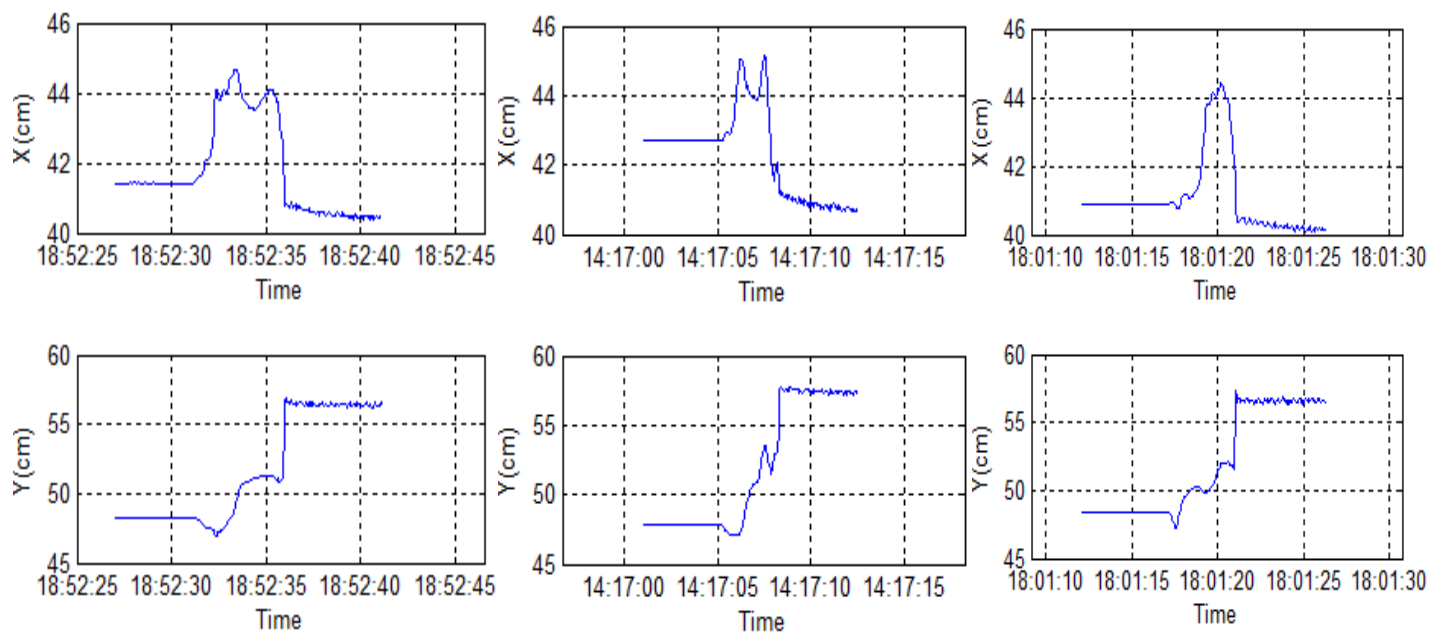

Figure 39: Three young people break-down COP plots of bed-exit.

\subsection{Discussion}

Comparing young people bed-exit COP plots with the senior's COP trajectory plots shown in subsection 4.3.1, the senior's plots show that there was plentiful small movements. Those small movements may be related to health related complications due to reduced muscle control ability and some noise. The data collected from these sensors is valuable to care givers and doctors because by using the algorithm mentioned in this chapter can provide a quick visual representation of participants' quality of sleep over a chosen period of time. In the future, the algorithm will need to be refined to make visual displays clearer for interpretation and user friendly for health care providers. Future work could also explore the use of a filter for this algorithm, helping to further reduce the work 
required to thoroughly analyze larger data files visual information of COP movement. The high sampling rate produced a sensitive and accurate display of COP movement. However, the use of a filter will allow related health care professionals or others to quickly produce plots that are able to pin-point various patterns associated with health trends. The results of this chapter reflected patterns observed in an elderly individual. Analysing more falls will make this result stronger. Among the seniors who participated, there were very few falls. Inpatients have caretakers, instruments and mainly they were staying inside so they also experienced very few falls during the study. The algorithm will allow healthcare workers to discover patients' patterns on the COP plots and health trends by observing bed exits transfers over time. 


\section{Chapter 5: Center of Pressure Magnitude}

\subsection{Introduction}

As described in Chapter 4, COP is the center location of all applied pressure. However, in addition to the location of COP, this research also examined the magnitude of the pressure applied to a mat for a further understanding of sleep patterns and health related issues during bed-exit. COP magnitude represents the amount of applied pressure at every COP location, revealing information about the amount of pressure a person applied to the mat coverage area. Accordingly, COP magnitude changes can show the bouncing problem when a person gets out of a bed. For instance, a patient who is having trouble getting out of bed may have to attempt the exit several times before succeeding and this creates a bouncing effect on the mat [42]. A typical cycle of bouncing during bed-exit often includes three periods, 1) lifting buttocks with a decrease of the total applied pressure on the bed, 2) sitting back with an increase of the total applied pressure on the bed and 3) lifting the buttocks again showing another decrease of pressure. The three periods happen in a short time interval. Bouncing can combine with an increase in sway to indicate information about a person's postural control ability. Information on pressure magnitude changes therefore can provide important information to health care providers on the amount of bouncing behaviours. This can serve as a red flag indicating difficulty 
getting out of bed and allow intervention to decrease the risk of a fall, which may lead to seniors injuries.

\subsection{Generating Magnitude Graphs and Video}

This section describes the algorithm for magnitude evaluation and how magnitude graphs and video may help researchers to know the movements of a subject before they get out of a bed.

\subsubsection{4-Dimensional graphs}

The algorithm for measuring COP magnitude took the sum of the active sensors' pressure values $^{3}$, then divided by the total number of active sensors. A COP location and a magnitude value can be calculated for every sample, then plot COP with magnitude together. To plot them together, magnitude was added as a $4^{\text {th }}$ dimension on the COP plot to display pressure magnitude with respect to $\mathrm{X}$ and $\mathrm{Y}$ directions and time. Each plotted dot shows the COP location by the dot location and pressure magnitude of that timestamp by the size of the dot. The change of pressure magnitude is indicated by the dot's color and size changes; see Fig. 40 as an example. Specifically, the biggest size of the dot displays the highest pressure, and the dot's size variation based on the applied pressure changes normalized with the highest pressure. The color changes follow the color scheme,

\footnotetext{
${ }^{3}$ It should be noted that the average pressure values used here are normalized to be in the range 0 to1. This is done to avoid varies that are caused by the absolute weight of the person.
} 
from dark blue to dark red represents 40 percent of the highest pressure to the 100 percent of the highest pressure.

When there is an increase in applied pressure on the bed, the magnitude of applied pressure is increasing, so that the size of the dot will increase and the color of the dot will change to the upper color in the color scheme to display the increased magnitude, as shown in Fig. 40. On the contrary, if there is a decrease in applied pressure on the bed, the dot will become smaller and the color of the dot will change to the lower color in the color scheme with the decreasing of the pressure magnitude. These magnitude dots are scaled to give a visual representation of the applied pressure variation in magnitude. In order to make the size of dot comparable between different individuals, normalized magnitude values were used. Using every pressure magnitude value divided by the maximum magnitude value of the whole data file got normalized magnitude. Otherwise dots size would vary with different weights of different people so that a large heavy person would have a very large dot and a small lift person would have a small dot.

The normalization process allows for reliable comparison of magnitude changes among people of different weights. Figure 40 is a sample 4-D plot of a bed transfer. It can be seen that dots' color and size are changing, displaying changes in the applied pressure. However, from this plot it can be seen that many of the COP dots are overlapping. The overlapping dots are difficult to be interpreted well and give limited information. 


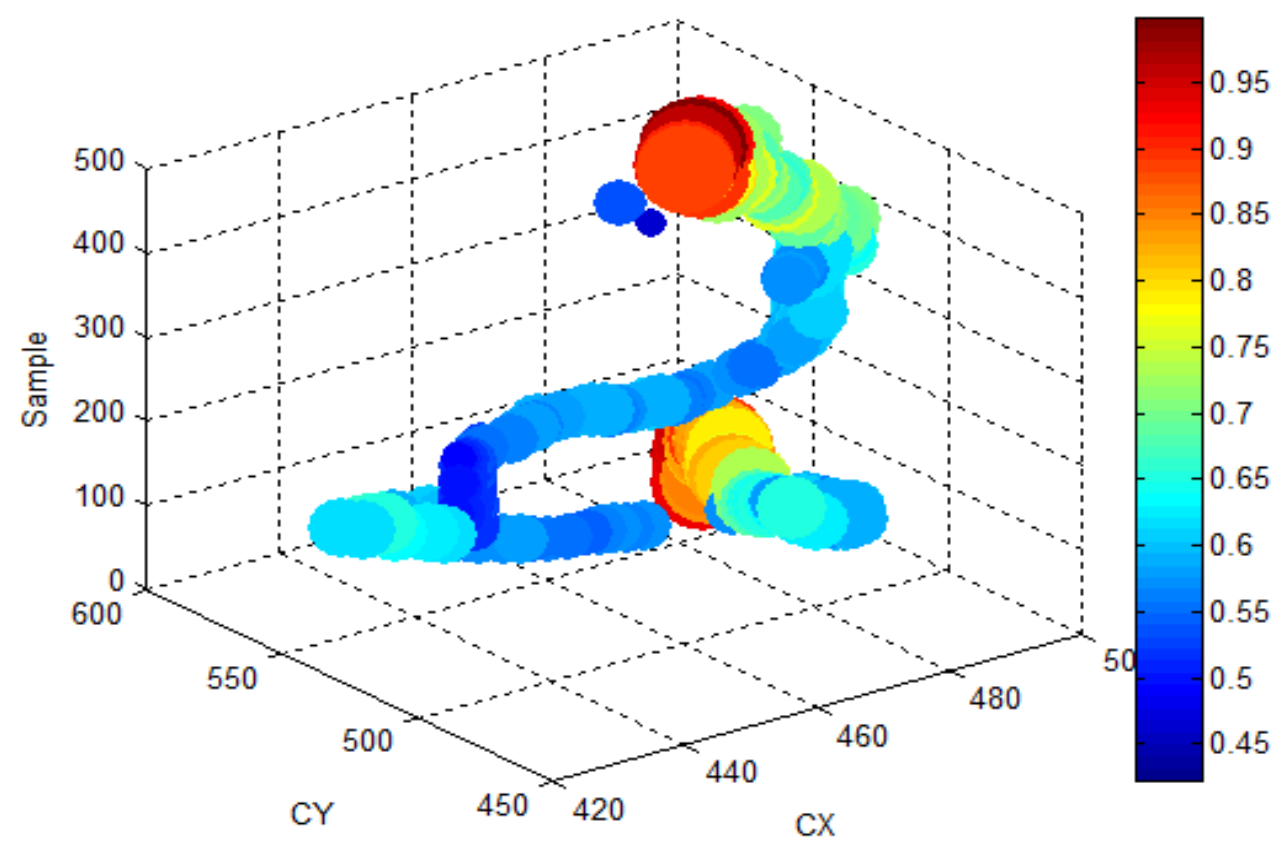

Figure 40: 4-D COP with magnitude scatter plot.

\subsubsection{COP magnitude video}

After considering the drawback of 4-D plots that overlapping dot gives limited information illustrated in the end of previous section, an improved method of graphical video display was developed as an alternative method of displaying four features: $\mathrm{x}$ direction, y direction, magnitude and time. Plotting a fixed area as main pressure coverage area of a mat, a dot on this area is the COP of a subject. The size of the dot shows the magnitude and the dot size changes as mentioned in subsection 5.2.1. The frequency of data was $20 \mathrm{~Hz}$, which meant there were 20 dots per second. A video was made up by displaying the dots, one after each other at the rate of $20 \mathrm{~Hz}^{4}$.

\footnotetext{
${ }^{4}$ Viewed as a video with a frame rate of $20 \mathrm{fps}$.
} 
An algorithm was written in MATLAB for this purpose. Using this algorithm, a magnitude video can be built. Figure 41 and 42 are screenshots from a video of an older participant's data file. The partial $\mathrm{x}$ and $\mathrm{y}$ axis of Fig. 41 and 42 shown in the video were chosen to display the main area of the whole mat. In general, a subject's COP dot do not move to a corner of the pressure mat, so displaying all the mat cover area is unnecessary. In Fig. 41 the dot size is much larger than the dot in Fig. 42, means that the participant reduced the pressure applied to the mat. From a sequential video, it is obvious how the location and size of the dot changes.

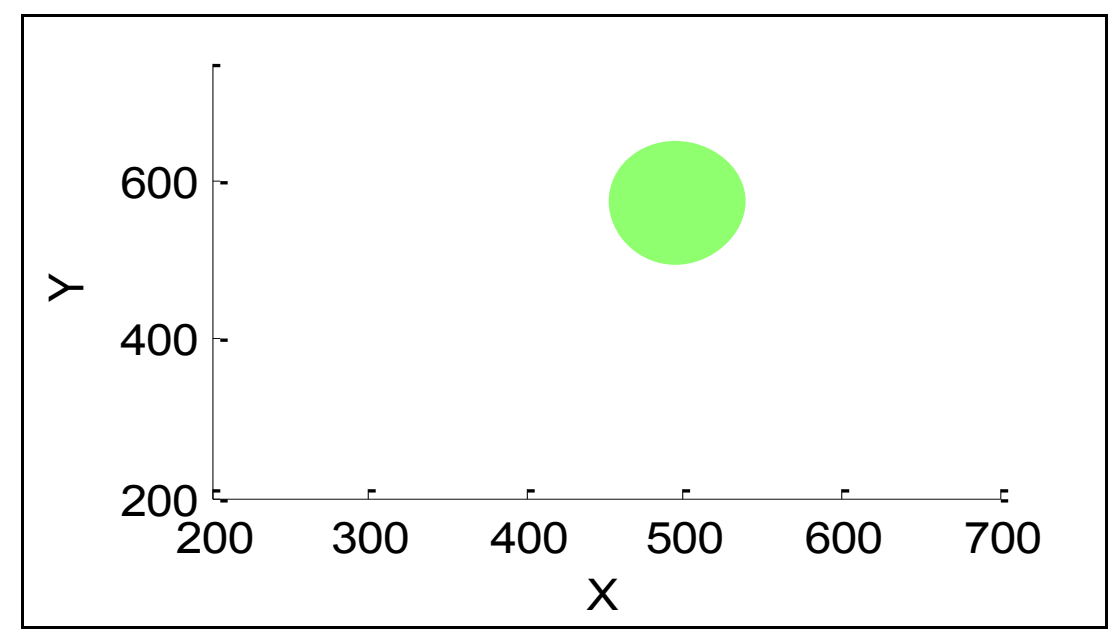

Figure 41: COP dot with a large pressure value (screenshot from a video). 


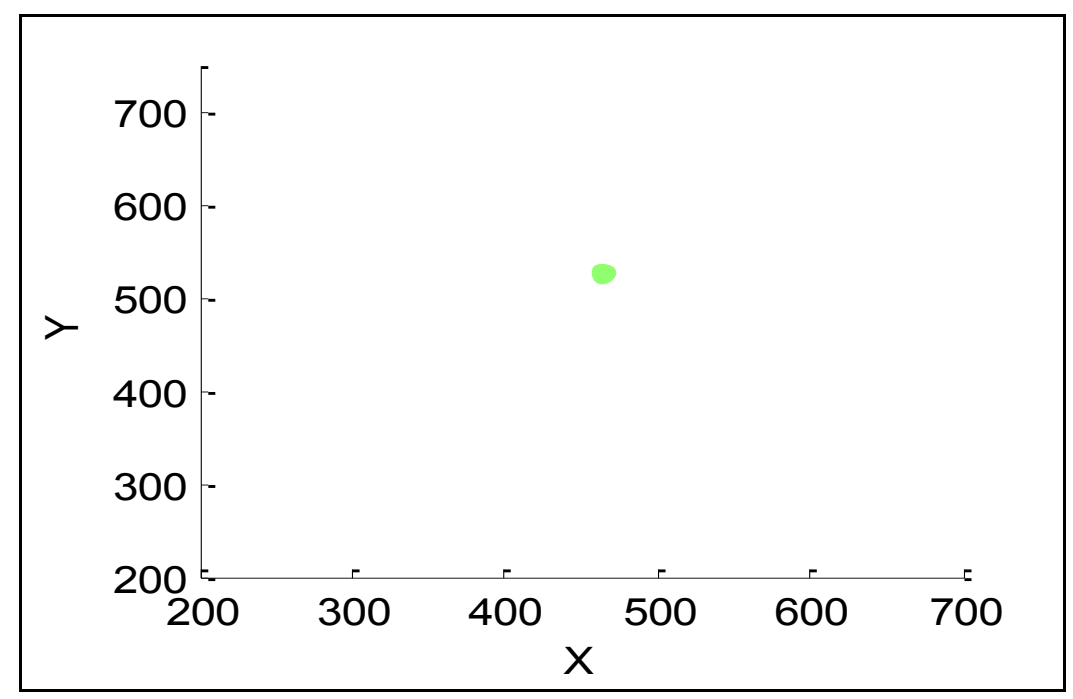

Figure 42: COP dot with a small pressure value (screenshot from a video).

From COP magnitude video, it can be seen how a person moves on the mat covering area, and how the amount of pressure applied to the mat changes. When a senior or a person who has balance problems gets out of bed, this pressure video can display extra effort required to do so. It has been observed that bouncing may also occur during that time [42]. As mentioned in the introduction section, bouncing is when a person lifts his buttocks, sits back down on the bed because of lost balance or other reasons and lifts hips again, which can be gained from the data as a pressure decrease, increase followed by another decrease. In some cases, bounces have been observed to happen many times during one bed-exit because of the subject's health situation. Accordingly, if a person had bounces during a bed-exit, doctors, caretakers or researchers can see them on the COP magnitude video. It shows by the dot size changing from big to comparable small to big then small again while the dot location moves from the middle to one side. 


\subsection{Results}

One advantage of the COP magnitude video is that it is easy to read, even for those who do not have a professional background in engineering or medicine. By viewing differences in dot movement and size, COP information is relatively easy to interpret. The route of dot moves on the video revealed how this person's center of pressure moved on the bed. If rocks happened during bed-exit, they can be found out. Rocking back and forth in the $\mathrm{X}$ direction shows that the location change in this direction only while movement in the $\mathrm{Y}$ direction would not change. Rocking movement in the $\mathrm{Y}$ direction would result in the dot traverse in only the $\mathrm{Y}$ direction while movement in the $\mathrm{X}$ direction would stay constant. The dot size variation can be used to observe different postures change and bed-exits. As an example, a person is changing posture from lying to sitting (more top body pressure is on mat to only the buttocks are on the mat) to standing if observed an increase in pressure followed by a large decrease of pressure since the dot will shrink out of view with a complete exit. As mentioned before, when the dot size becomes smaller and bigger and then smaller again during a bed exit, this pattern is typical of bouncing. Besides those, the COP video can also be used to monitor the time that it takes a person to get out of bed. Bed exit timing is another important indicator of a person's mobility which is hard to decide of the starting point so we will not focus on timing. 


\subsection{Discussion}

This research found that in young healthy volunteers bed-exits were smooth, fast and free of other small movements typical of rocking and bouncing by observing the bed-exits magnitude videos. It also found that in the older volunteer, there was more size changes and small dot magnitude movements on the bed-exits pressure magnitude videos. Since there are a large number of seniors living alone and age is associated with a higher risk of mobility decline and health problems, this method of monitoring bed exit mobility may represent an important tool in the future. Long-term monitoring of their health situation becomes an essential method of ensuring their safety. It is relatively unobtrusive and gives health care providers a way of monitoring seniors in their home environments. In this way, the technology and algorithms can contribute to preventing more serious health problems by picking up early signs of difficulty. 


\section{Chapter 6: Center of Pressure Dynamic Behaviour}

\subsection{Introduction}

This chapter discusses how the instantaneous speed ${ }^{5}$ and the rate of change of speed $(\text { ROCS })^{6}$ of COP were calculated. The chapter includes a discussion of methods of decreasing noise. The noise recorded in the data and introduced a limitation to accurately measure speed and rate of the change of speed.

There are three ways to improve the quality of data: thresholding, averaging and filtering. Since threshold was used from the beginning, so we will only look at averaging and filtering here.

\section{Explanatory note}

Velocity contains two characteristics, the first is a subject's movement speed between positions and the second is the direction of motion; combining these two features is the velocity vector [70]. If there is a change of speed or velocity direction, the subject is observed to be accelerating. Acceleration is used to display the rate at which a subject's velocity changes with respect to time [70].

\footnotetext{
${ }^{5}$ Also referred to as velocity magnitude in this thesis.

${ }^{6}$ In this thesis we are only concerned with the magnitude of the velocity vector, and the rate of change of this magnitude.
} 
When a subject's motion velocity magnitude (not include the direction of motion) increases, acceleration is positive; when a subject's motion velocity magnitude decreases, acceleration is negative. The value of acceleration depends on the magnitude of the velocity changes. Acceleration is defined as the rate of change of velocity.

Velocity magnitude and ROCS calculation by COP location with time was used to translate a visual representation of how participants perform of bed-exits in this chapter. Averaging and filtering were used to modify the velocity plot and they will be discussed at this chapter.

\subsection{Algorithm for Calculating Velocity Magnitude}

A number of 72 sensors bed pressures mats (include type-1 and type- 2 mats) were placed under participants' mattresses, and collected pressure data continuously. Chapter 5 discussed the process of locating and calculating the center of pressure. This section will expand on the method of computing COP velocity magnitude (instantaneous speed) by using COP locations.

The participant COP movement velocity was calculated based on the change of the locations of the COP versus time. The locations were used to calculate the COP displacement between desired time intervals. The displacement is then divided by the time interval to calculate velocity magnitude (speed). The direction of that movement can 
also be calculated from the start and the end locations. COP locations expressed by coordinate values based on the coordinate axis setting of the pressure mat. From the difference of coordinate values between the start and the end point, movement angle $\theta$ can be calculated by using equation (3).

The Type-1 mats have a data logging frequency of $20 \mathrm{~Hz}$, meaning that every second there were $20 \mathrm{COP}$ points recorded, so that every two sequential points have a time difference of $1 / 20$ second. For example, time between COP point one $\left(P_{1}\right)$ and COP point two $\left(P_{2}\right)$ was $1 / 20$ seconds, likewise the time between point two and point three was $1 / 20$ seconds, etc. The time interval for the velocity calculation was simply correspondingly changed with the number of COP points were used. Figure 43 is an example of the distance between adjacent points, from point $P_{1}$ to the next point $P_{2}$, where $\mathrm{X}$ and $\mathrm{Y}$ direction values were $(\mathrm{x} 1, \mathrm{y} 1),(\mathrm{x} 2, \mathrm{y} 2)$ respectively. The distance between $P_{1}$ and $P_{2}$ was found by summing the $\mathrm{X}$ and $\mathrm{Y}$ direction distance difference of squares and then taking the square root of it, as shown in equation (1). The magnitude of the velocity is the moving rate over time, is calculated as equation (2), that distance divided by time. The direction from $P_{1}$ to $P_{2}$, equation (3) shows $\theta$ is the angle from $P_{1}$ to $P_{2}$. If more than two points were being used to calculate velocity magnitude, the distances between each sequential two points must be summed. This chapter will focus on observing the movement speed patterns rather than specific direction of the movement.

$$
\begin{aligned}
& r_{2}=\sqrt[2]{(x 2-x 1)^{2}+(y 2-y 1)^{2}} \\
& s_{2}=\frac{r_{2}}{t}
\end{aligned}
$$


$\theta=\tan ^{-1} \frac{y_{2}-y_{1}}{x_{2}-x_{1}}$

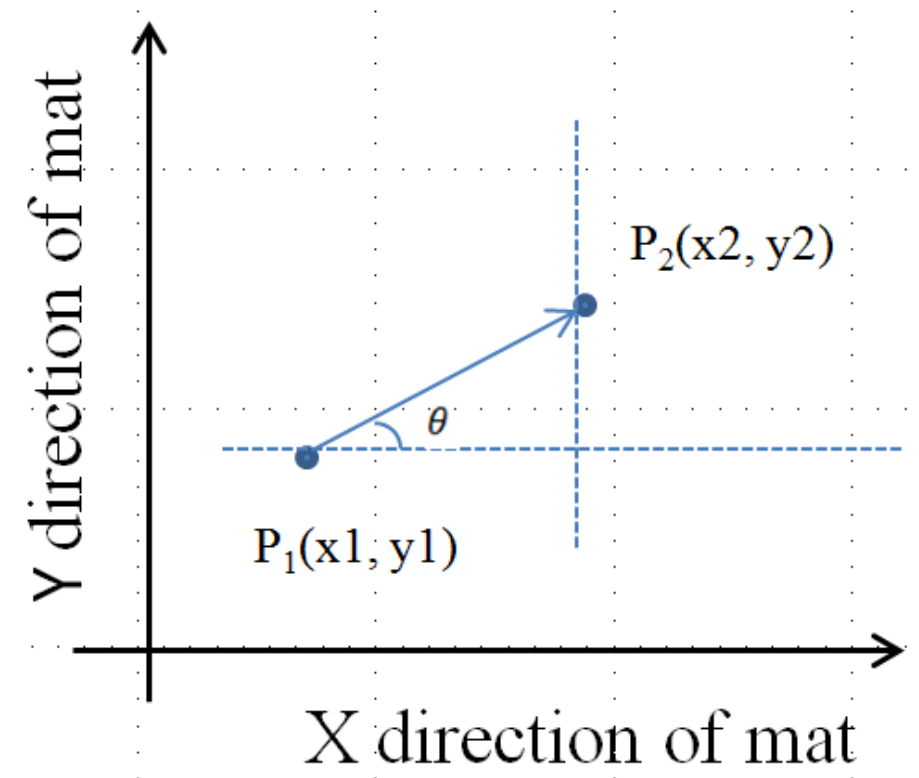

Figure 43: Two COP locations on mat coverage area.

This algorithm of instantaneous speed, versus time, was tested by doing experiments at a laboratory system set-up. A subject sat on the bed, leaned slowly to the left then slowly to the right, and then she moved relatively fast to the left and right (pictures in Fig. 44). Data collected from the test was processed by the proposed algorithms. Figure 45 is the velocity magnitude (speed) plot, Fig. 46 is COP-X direction and COP-Y direction plots, and Fig. 47 is COP trajectory plot. From Fig. 45, T1 was when the subject sat on the bed fast, T2 and T3 were the stages she slowly moved to left then right, T4 and T5 shown she leaned to left then right relatively fast, T6 was she got out of the bed. The velocity changes and differences could be seen from velocity plot Fig. 45 and trajectory plots Fig. 46. At Fig. 46, the angles of slopes for T4 and T5 were much lower than T4 and T5's 
slopes which were nearly-vertical. From the slope we can also obtain the conclusion that T2 and T3 indicated movements which were slower than T4 and T5 indicated movements. Figure 47 was a COP trajectory plot of this test, and it could reveal the velocity changes from timing of every movement and slope. The movements with different velocities can be seen at the video and the changes matched with Fig. 45-47. That verified the algorithm for velocity.
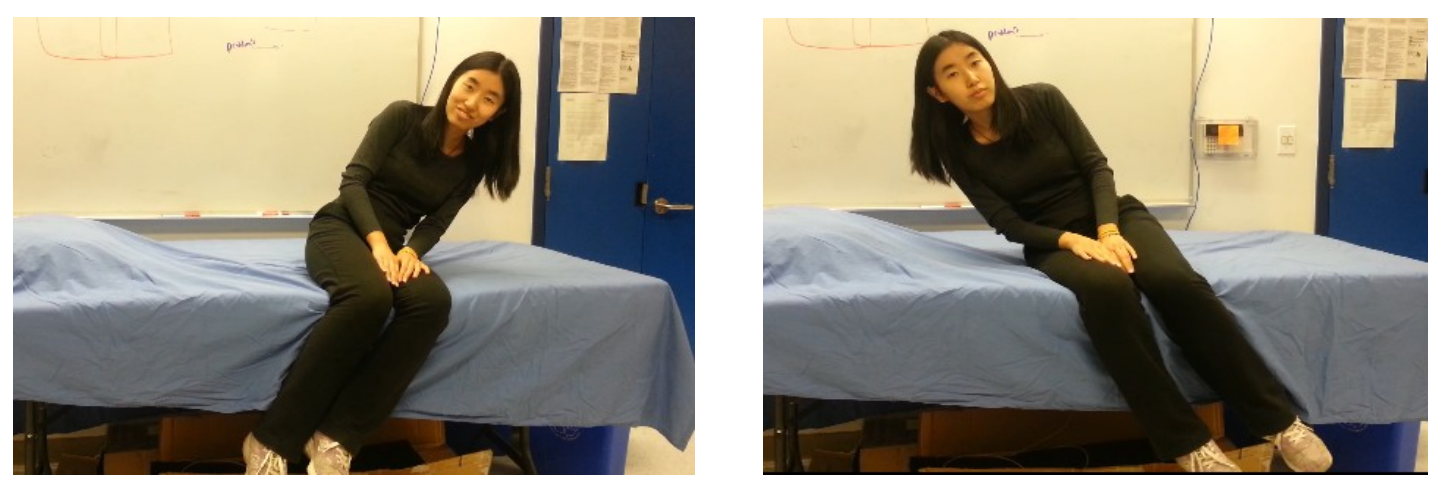

Figure 44: The subject leans to her left and her right with different speed for lab test of velocity. 


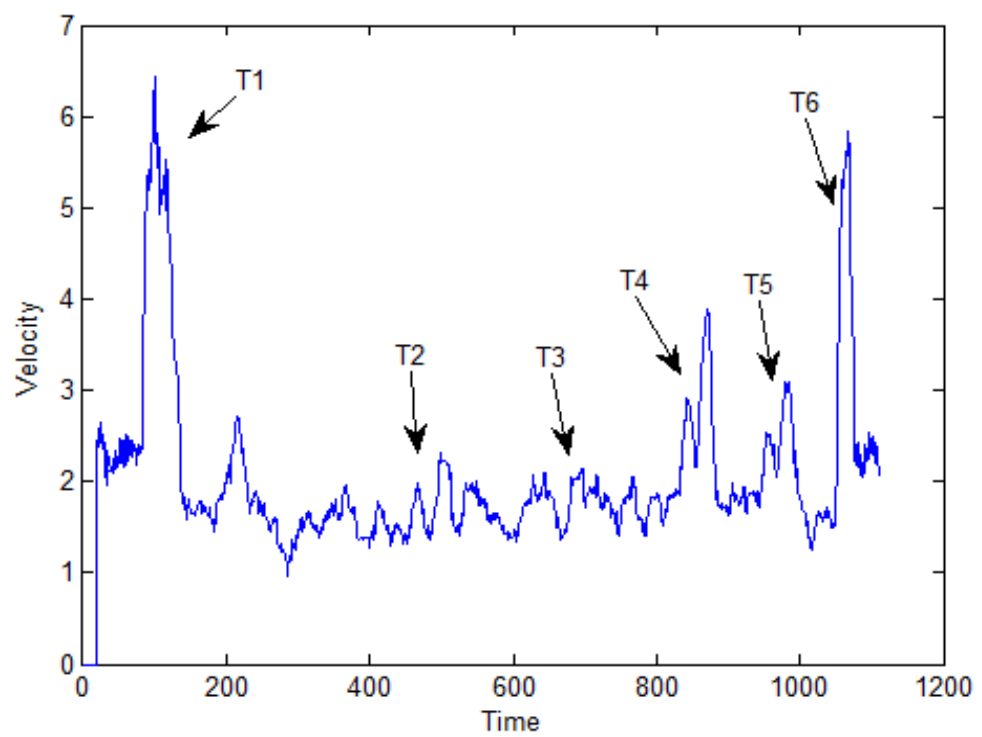

Figure 45: Slow and fast velocity plot versus time (in sample intervals) from lab test showing on magnitude.
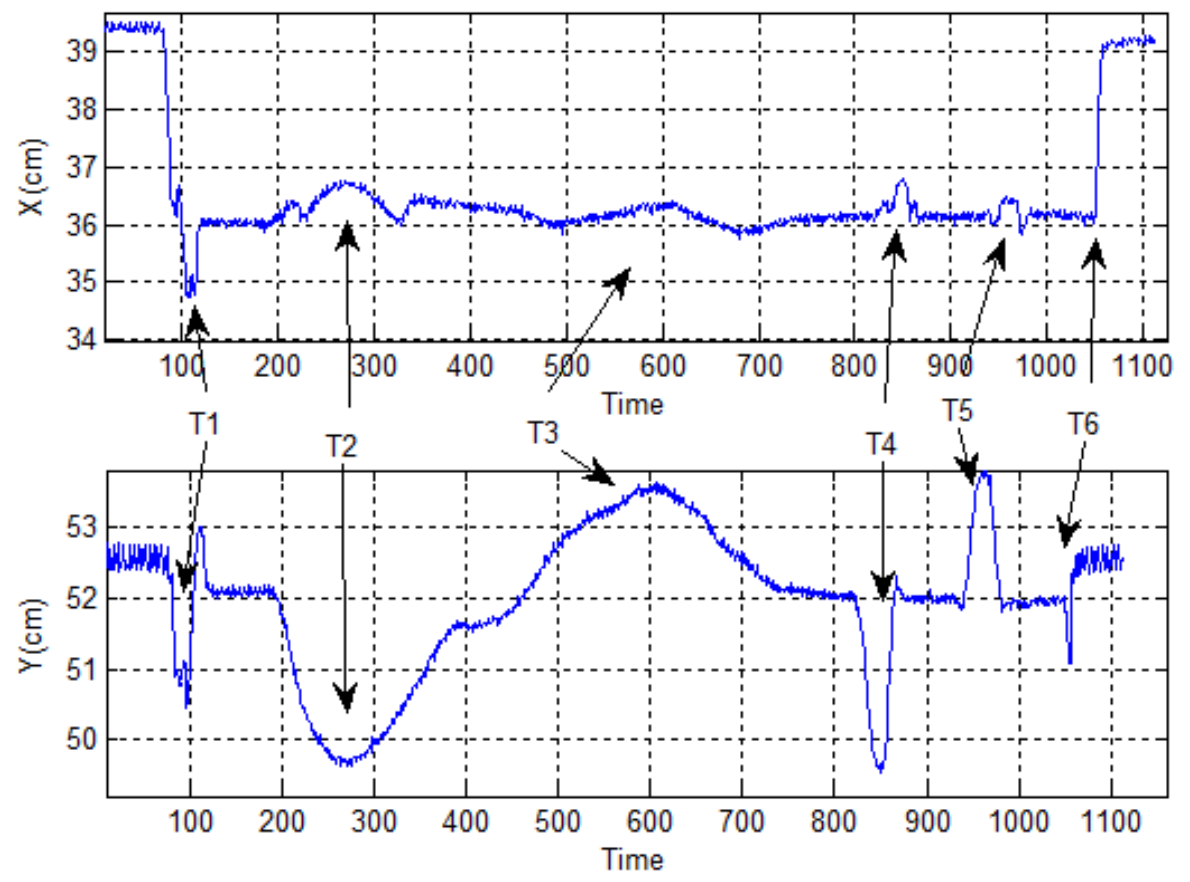

Figure 46: t-X and t-Y plots versus time (in sample intervals) from lab test. 


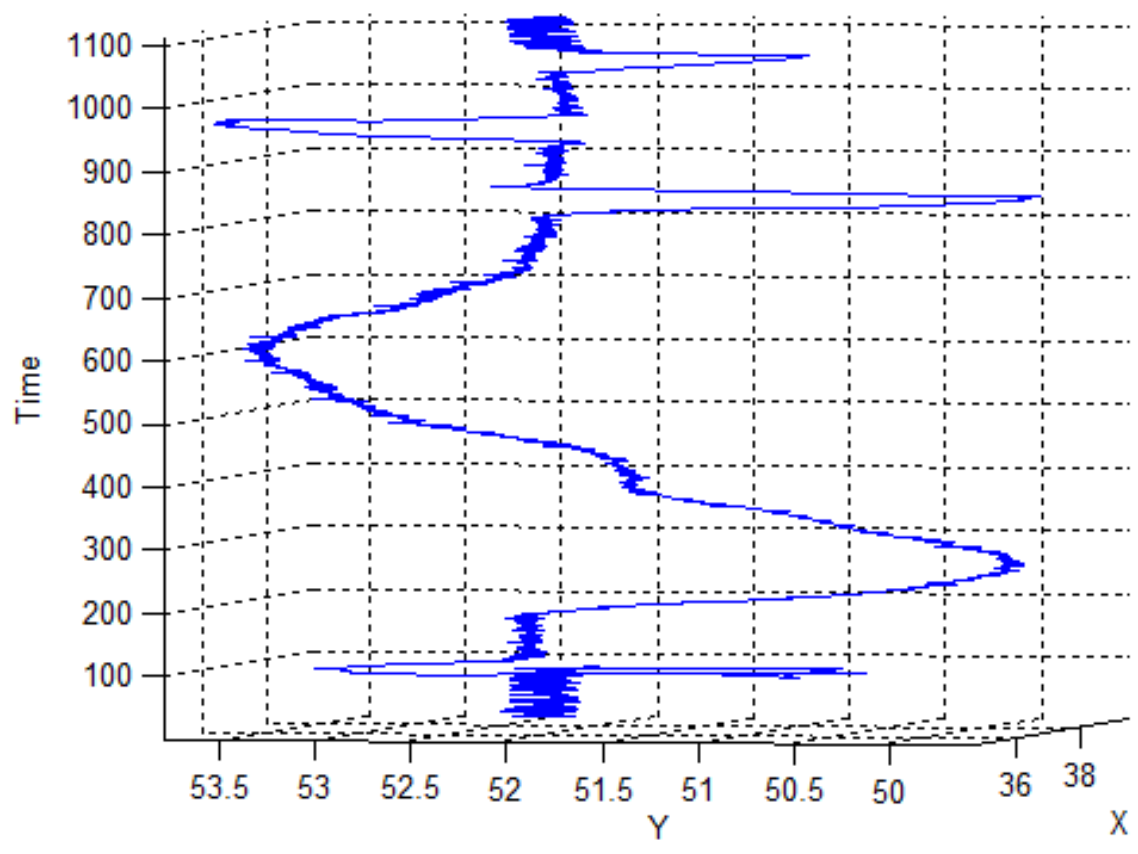

Figure 47: COP 3-D trajectory plots from test (used "Rotate 3D" function to make the plot readable).

\subsection{Noise Suppression in the Computed COP Velocity Magnitude}

There is always a small range of random noise in the sensors pressure value, even under fixed load condition [71]. Methods of reducing noise in order to display clean velocity plots will be discussed in this section for producing an accurate interpretation of movement velocity magnitude. 


\subsubsection{Using 2 points of COP location}

COP velocity magnitude could be obtained using two consecutive locations of the COP. As section 6.2 explained of the method of determining velocity magnitude, processing two sets of coordinate values from two consecutive points helped us get the COP velocity magnitude. We focused on the magnitude of the velocity only.

This method of COP velocity calculation was used on older participant data during bedexits to produce time-velocity plots, with time on $\mathrm{x}$ axis and velocity magnitude on $\mathrm{y}$ axis. The velocity plot values were always greater than zero when direction is not accounted for. Figure 48 shows the velocity change between two successive COP location points (V2) ${ }^{7}$, was using the same data file as Fig. 24, in Chapter 4. After looking at many time-velocity plots, they appeared noisy. The changes were mainly caused by random noise of pressure sensors. . When the noise add up with a participant's actual movement velocity, it makes a plot have small rapid fluctuations. Moreover, this pressure sensor mat is sensitive on pressure changes that even very minor speed changes can be captured. We needed to reduce the noise effect on velocity magnitude.

\footnotetext{
${ }^{7}$ The ' $\mathrm{V} 2$ ' indicates the instantaneous values obtained from 2 consecutive COP locations later on we will use 'V10' and 'V20' to indicate average speeds over 10 and 20 consecutive COP locations.
} 


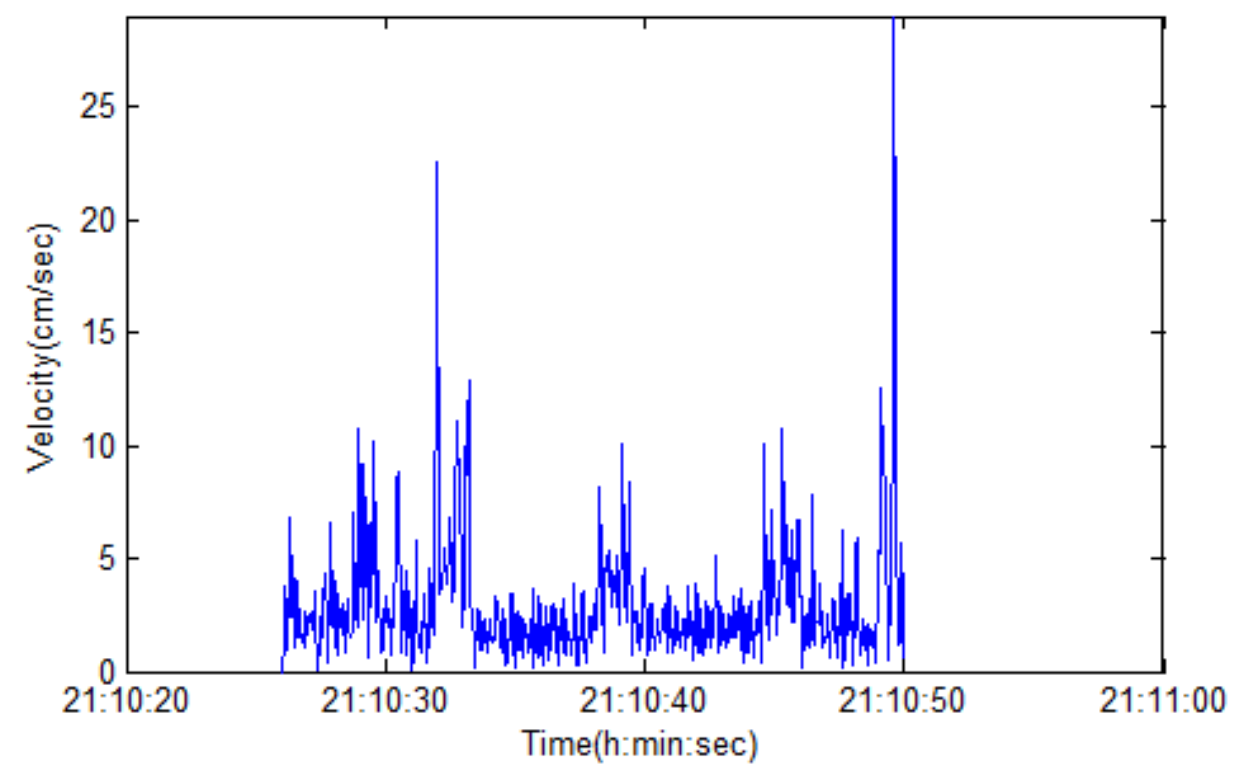

Figure 48: Velocity magnitude plot calculated by every two adjacent COP locations (V2).

\subsubsection{Using 10 points of COP location}

From subsection 6.3.1, we know that using 2 points of COP location to calculate velocity magnitude is too sensitive since it captures all the small changes which may or may not be a subject's movements related. The way to decrease the effect of noise and smooth the plot is averaging. Specifically, averaging is using a window to average distances of several successive velocity magnitude values, then moves the window to the next sample. However, using a wider averaging window to get the mean velocity magnitude may cause a subject's slow movements to not be seen on a velocity magnitude plot. A proper window size is chosen as a compromise between its noise suppression potential and the potential suppression of slow movements in the velocity magnitude plot. 
One way to check the proper number of maximum points needed to average velocity magnitude is by considering the slope of movements in time- $\mathrm{X}$ and time- $\mathrm{Y}$ plots. Searching for the slowest movement which has the smallest slope in both plots, and then counting how many COP points were representing the slowest movement at both plots, they should have the same amount of COP points. To be able to see the slow movements, the amount of samples contained in the slowest movement need to be bigger than the averaging window. The level of slowness can vary, so that the slowest movements of different people especially between young people and older people can be very diverse. This means the numbers of COP points for slow movements is located in a wide range. To ensure this method could capture the small movements from both young and older people, the number of COP points included in slow movement of older people was used as a standard ${ }^{8}$. Overall the number of COP points in slow movements is higher than 20.

\footnotetext{
${ }^{8}$ This choice leads to results that were better perceived by the health care clinicians.
} 


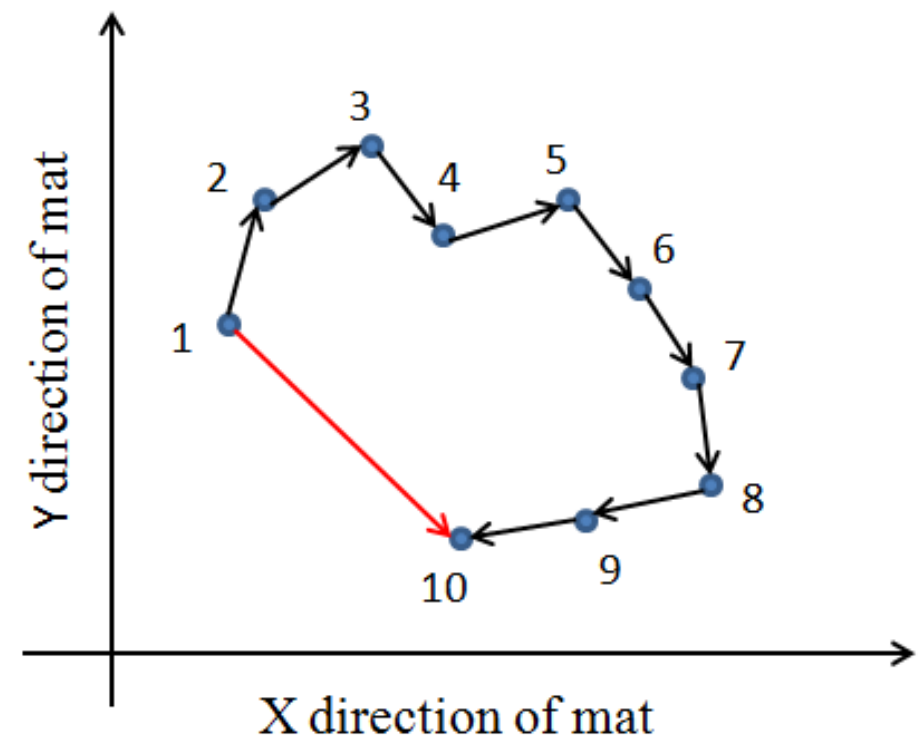

Figure 49: Two ways to calculate 10 COP points distance.

In subsection 6.3.1, velocity from 2 points of COP has been discussed. In this section we are going to talk about velocity from 10 points of COP. The way of getting 10 points average velocity is similar to using 2 points. If we were to use the start point and the end point to calculate the velocity, it will not be accurate because one subject may move around so the end point is close to the start point. This scenario the displacement from the start point to the end point would not reveal the whole path distance as shown in Fig. 49. There are 10 random dots on the plot to simulate a subject's consecutive COP locations represented by dots. The dot occurrence sequence follows the black arrows. If we were to just look at the first dot labelled 1 and the last dot labelled 10, it would be the wrong distance as shown by the red arrow on the plot. To avoid looking at only start and end points and to calculate the velocity value accurately we must use all 10 points, from the 
start point $\mathrm{P} 1$ to the end point $\mathrm{P} 10$ and 8 points in between $\mathrm{P} 2, \mathrm{P} 3 \ldots$ P9. The timing consumption of 10 points is 9 periods; each period between two points is $1 / 20$ second as mentioned before, so that 9 periods is $9 / 20$ second. The total distance from P1 to P10 is obtained by calculating the distance between every two close points and then adding those distances together. The way of calculating distance between two points is described in subsection 6.3.1. Using the total distance divided by the timing consumption which is 9/20 second produces a 10 points average velocity. For obtaining next COP velocity, the 10 points window slides one point towards the end from last window. Calculating velocities magnitude for the rest of data by using the described way, every time moves the window one point as shown at Fig. 50. The speed of velocity from P1 to P10 is $v_{10}$ :

$$
v_{10}=\frac{d}{t}=\frac{\sum_{i=2}^{10} \sqrt{\left(x_{i}-x_{i-1}\right)^{2}+\left(y_{i}-y_{i-1}\right)^{2}}}{9 / 20}
$$
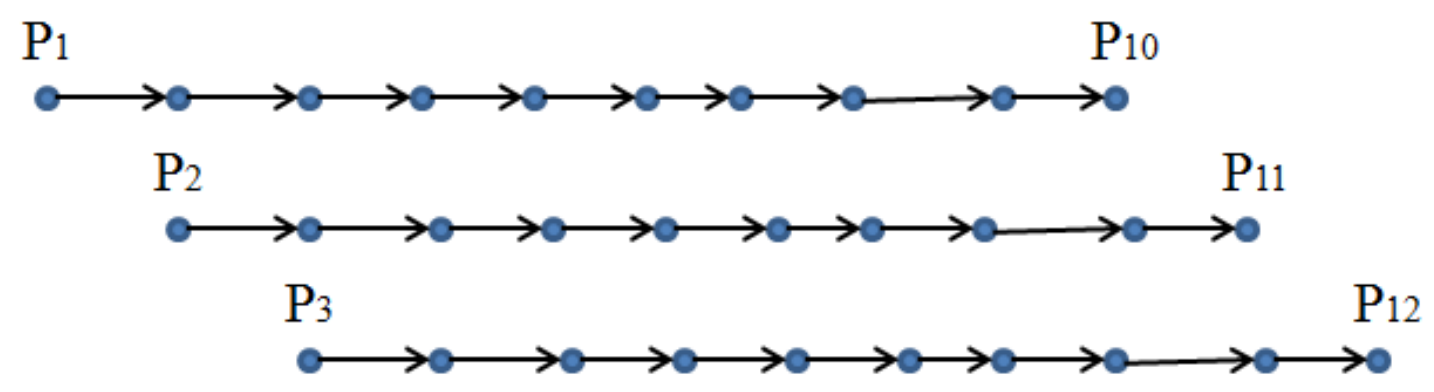

Figure 50: 10 points window. 
A MATLAB program was written to implement this algorithm. The program was run with participant data files. The resulting plots were smoother than those velocity plots generated by using successive two COP points. However, noises continued to cause small continuous fluctuations. That meant we needed to increase the averaging of COP point number to find velocity plots with less noise effect. Figure 51 shows velocity plots created by using 10 adjacent points, and used the same data file as with Fig. 48.

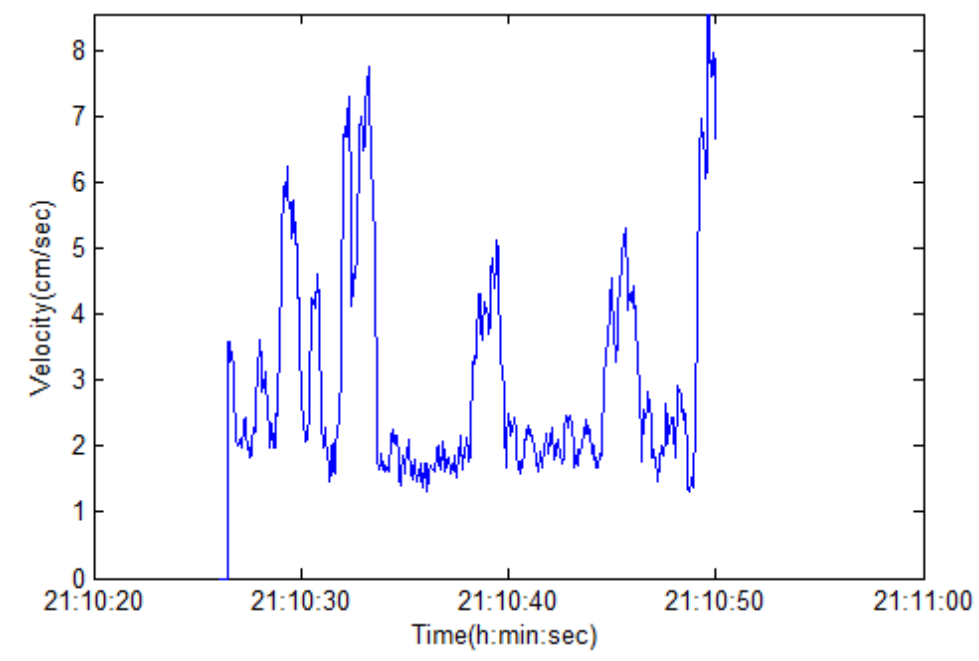

Figure 51: Velocity plot calculated using ten adjacent COP locations (V10).

\subsubsection{Using 20 points of COP location}

We described 2 points and 10 points of COP location to calculate velocity magnitude but later discovered that using $20 \mathrm{COP}$ points to calculate velocity was better and did not lose any slow movements from averaging. To get 20 points average velocity, we used 20 COP locations to calculate one velocity, then moved the 20 point window one point to the timestamp's larger side which was also the side of the end, until the end of a data file. 
The timing consumption from the first COP point $\mathrm{P} 1$ to the last point $\mathrm{P} 20$ in this window was 20 periods. Each period was $1 / 20$ second, so 20 periods (count in zero to P1 period) was 1 second. The total distance from P1 to P20 combined all the small segments of the distance between each successive two points, and then the total distance was divided by time consumption to generate a 20 points average velocity magnitude, the equation for this calculation is below:

$$
v_{20}=\frac{d}{t}=\frac{\sum_{i=2}^{20} \sqrt{\left(x_{i}-x_{i-1}\right)^{2}+\left(y_{i}-y_{i-1}\right)^{2}}}{20 / 20}
$$

Plots were much clearer and smoother compared with the 10 point average velocity plots. Figure 52 is velocity plot based on a 20 points window (same data files that were used for Fig. 48 and 51).

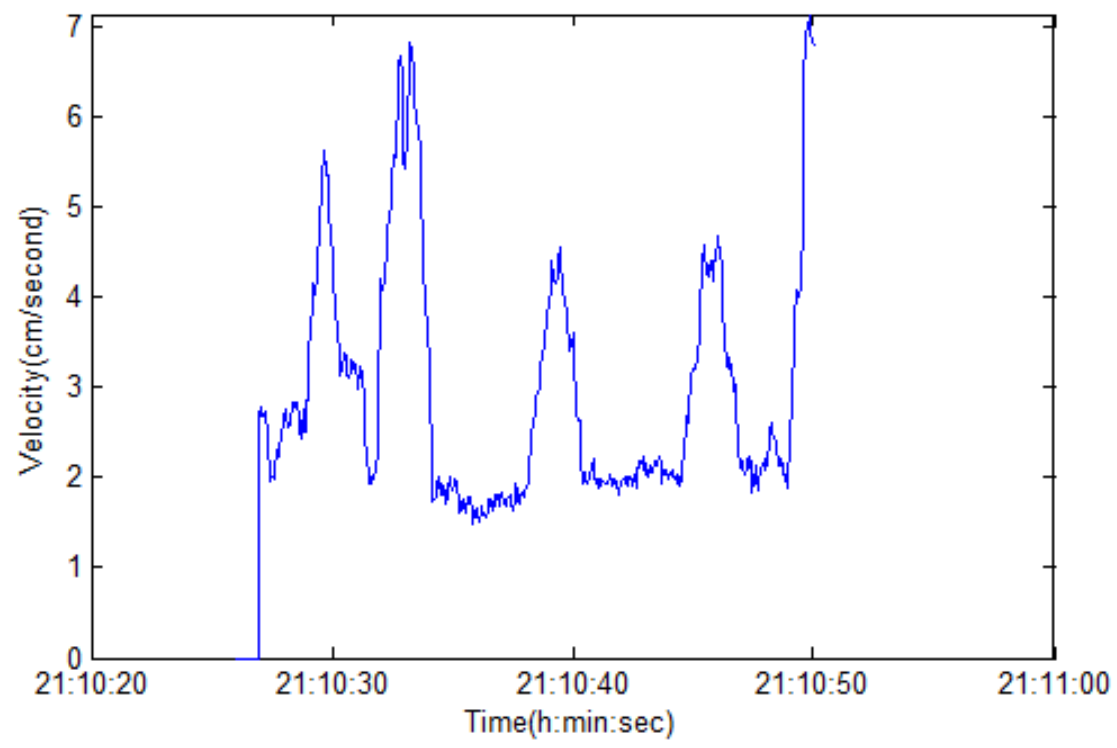

Figure 52: Velocity plot calculated by twenty adjacent COP locations (V20). 


\subsubsection{Using filter}

There is always delay by using moving average window. To get a zero delay signal, processing input data in both the forward and reverse directions [72]. To be consistent with other subsections in Chapter 6, used 10 samples and 20 samples as windows separately, the numerator coefficients of the filter was $1 / 10$ and 1/20 respectively. After filtering the data in the forward direction, reverses the filtered sequence and runs it back through the filter. Velocity magnitude with 10 samples window zero delay filtered signal plot is shown as Fig. 53. The 20 samples window zero delay filtered signal plot shown as

Fig. 54. The 20 samples moving window operation follows equation (5).

$$
v_{\text {filter }}(z)=v(0)+v(1) z^{-1}+v(2) z^{-2}+\cdots+v(19) z^{-19}
$$

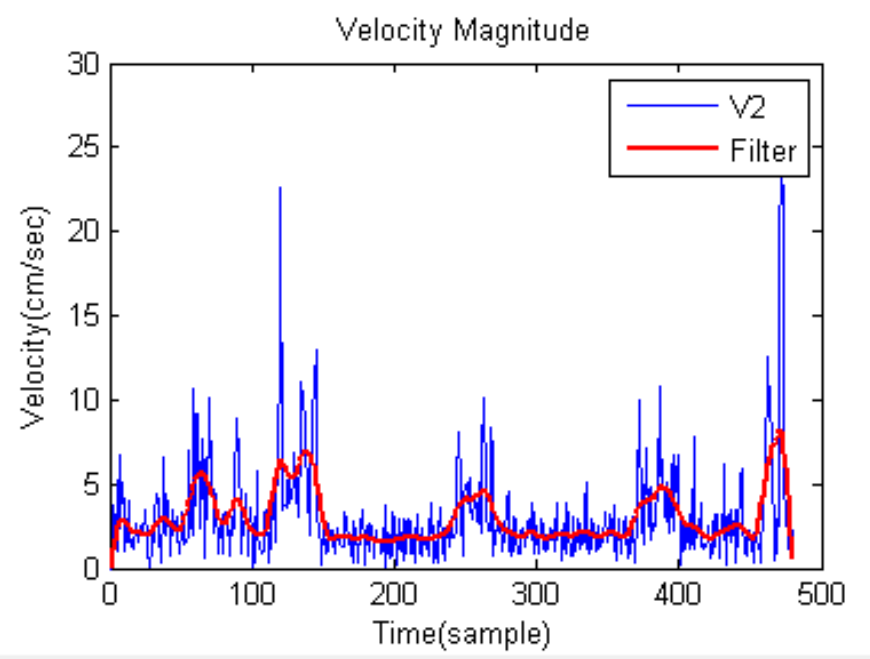

Figure 53: Window 10 filtered velocity magnitude plot with V2. 


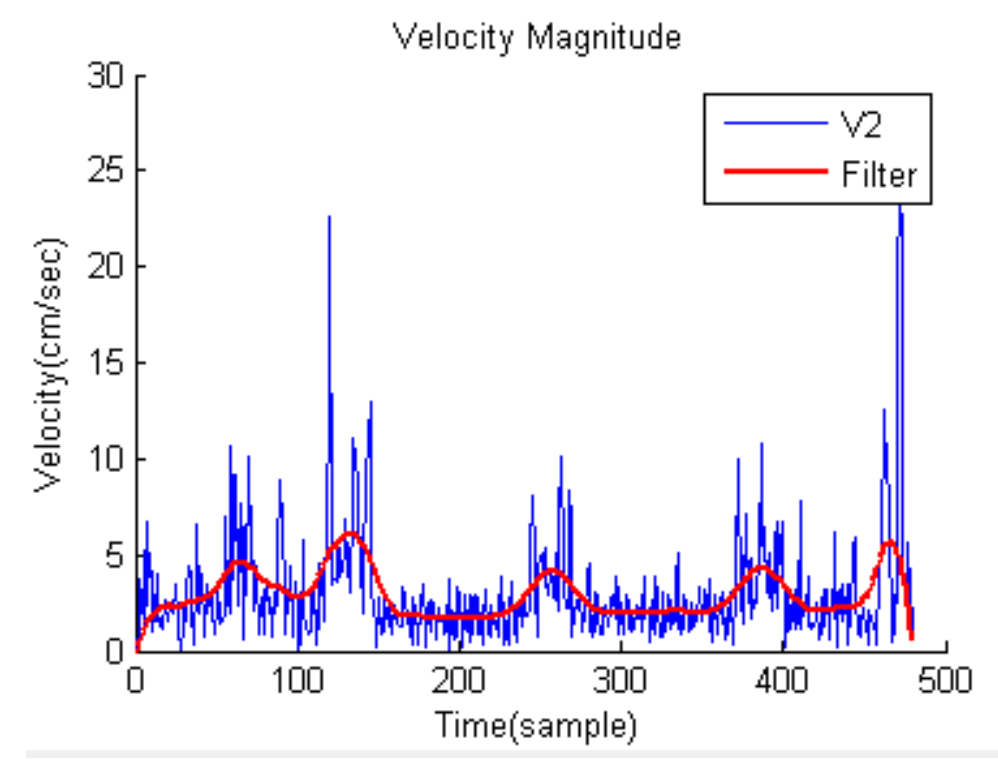

Figure 54: Window 20 filtered velocity magnitude plot with V2.

\subsection{Algorithm for Calculating Rate of Change of Velocity Magnitude}

\subsubsection{Rate of change of velocity magnitude from V2}

Acceleration was calculated from velocity and is velocity's change over time. In this thesis we are only concerned with the velocity magnitude and its rate of change. The change of velocity magnitude over time is the difference in velocity magnitude, in two consecutive COP locations, divided by the sampling interval $1 / 20$ second. A MATLAB program was written to calculate the rate of change of velocity magnitude and plot it. Figure 55 is a velocity magnitude change plot from V2 (used same data from Fig. 48). 


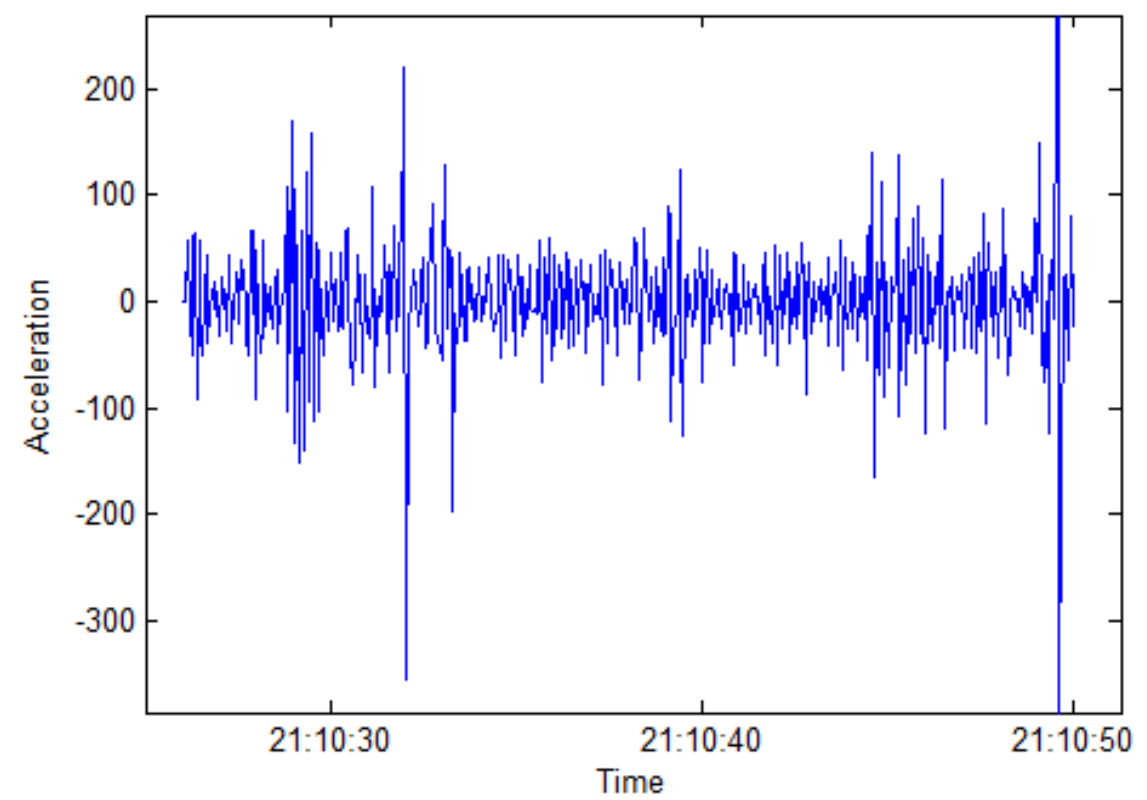

Figure 55: The change of velocity magnitude plot based on V2.

\subsubsection{Rate of change of velocity magnitude from V10}

The rate of change of velocity magnitude from the V10 plot was calculated using the method described in section 6.4.1. (i.e. using velocity values from V10). The plot below in Fig. 56 shows the rate of change based on a 10 point average (related graph: Fig. 51). 


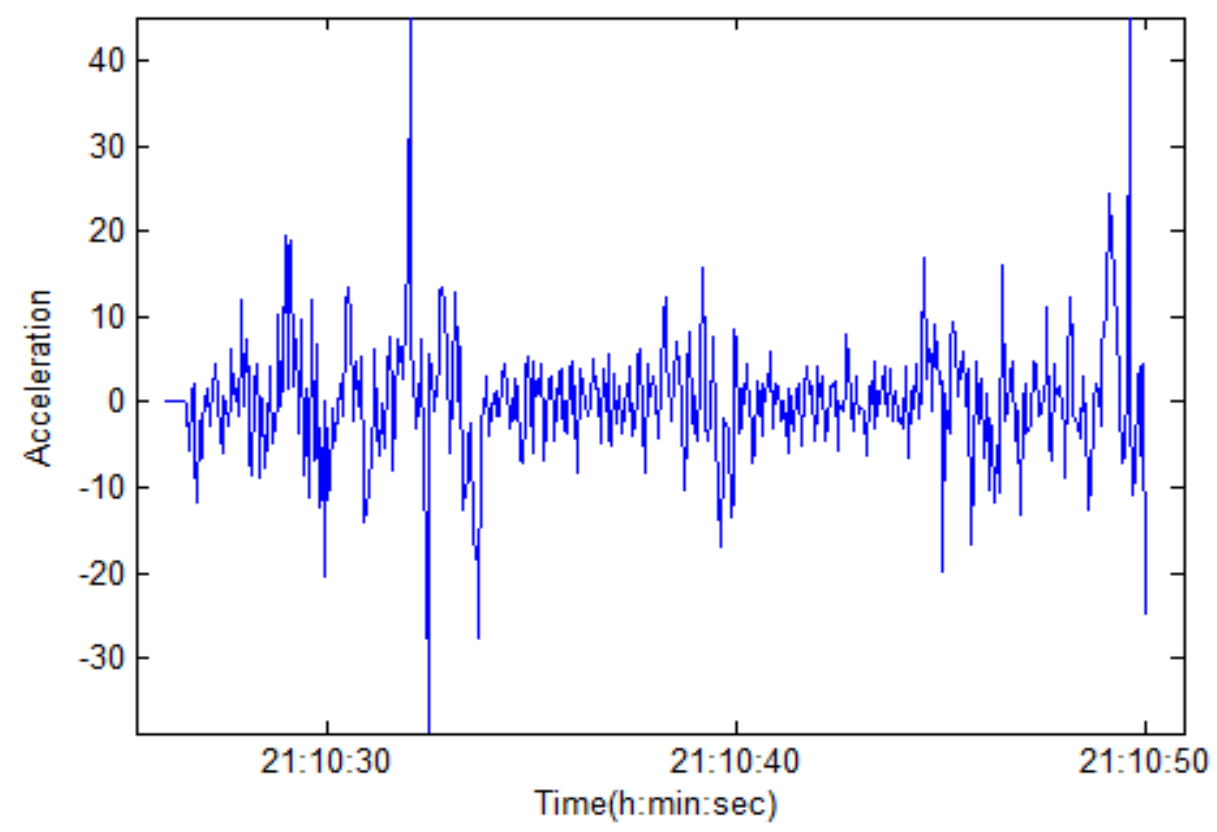

Figure 56: The change of velocity magnitude plot based on V10.

\subsubsection{Rate of change of velocity magnitude from V20}

Rate of change of velocity magnitude from the V20 plot was calculated with the method described in section 6.4.1. The next plot Fig. 57 shows the rate of change based on 20 points averaging (related graph: Fig. 52). 


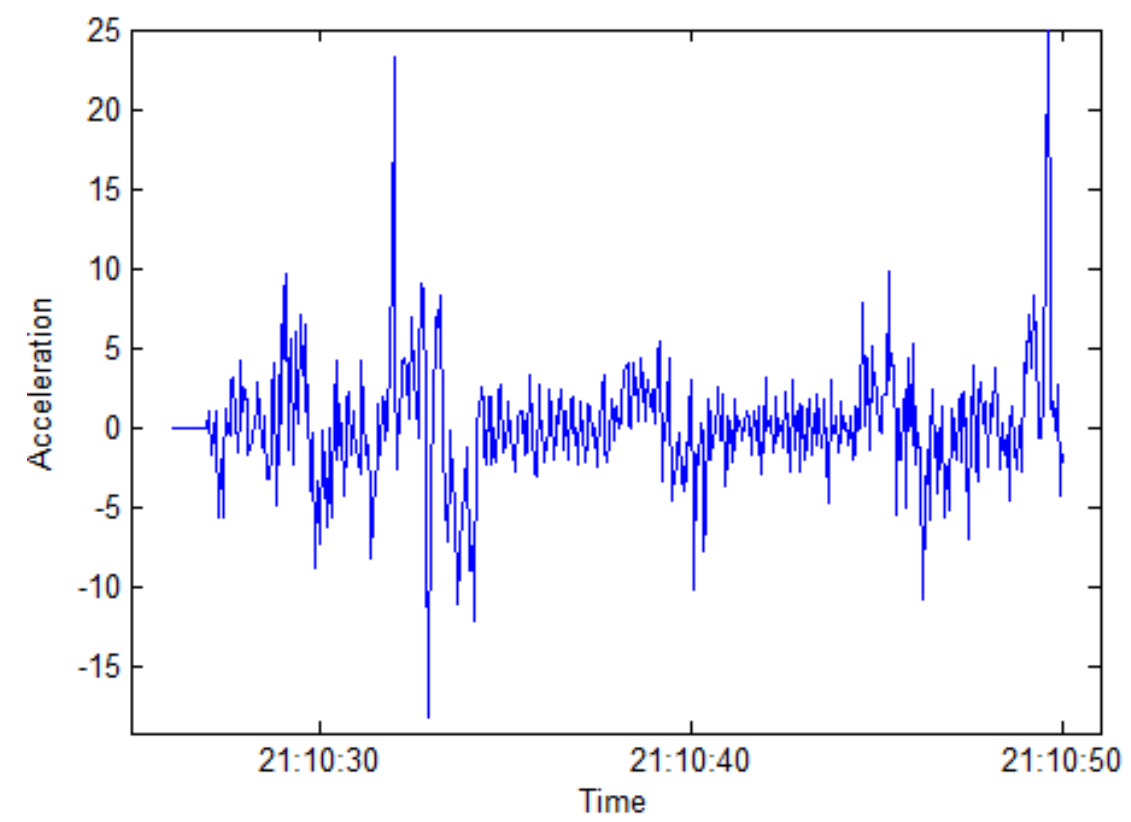

Figure 57: The change of velocity magnitude plot based on V20.

\subsection{Results}

Among all the separate plots of velocity magnitude and the change of velocity magnitude from different numbers of COP locations, putting some of them in the same plot so that we could see the difference clearly and commit a comparison between them. Figure 58 has two velocity magnitude plots; one is calculated by two COP locations (red), the other one is calculated by 10 COP locations (blue). The blue line has basically same peaks and valleys as the red line but without the outliers and with less noise interferences. In Fig. 59 , the red line is velocity magnitude with window of two COP locations, and the blue line is velocity magnitude with window of twenty COP locations. It is apparent that the V20 blue line is tremendously smooth and much better than the V2 line. For Fig. 58 and 59 the blue lines used moving averaging windows by 10 samples and 20 samples 
separately. Therefore, the delay was $10 / 2$ and 20/2 samples respectively. For compensating the delay of averaging window, time-shifted the input signal. From the plots we can see the averaging window did help to decrease the noise of data processing.

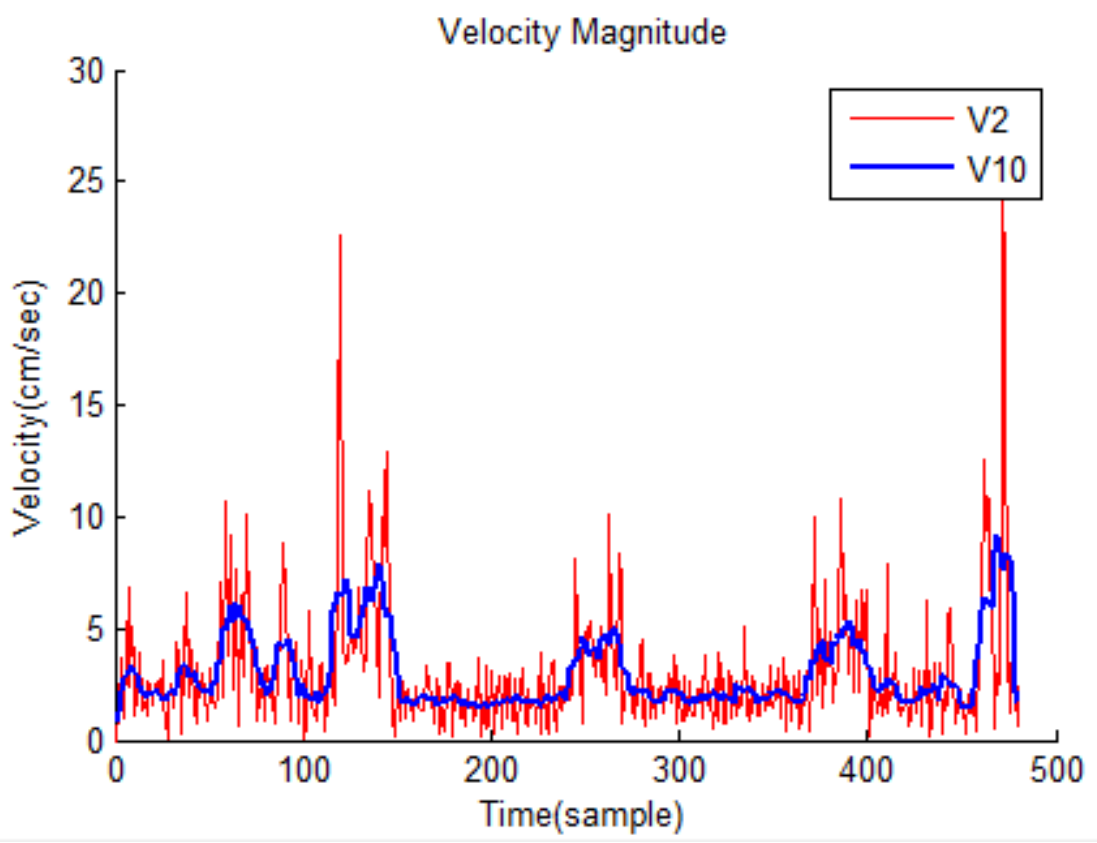

Figure 58: V2 and V10 comparison plot. 


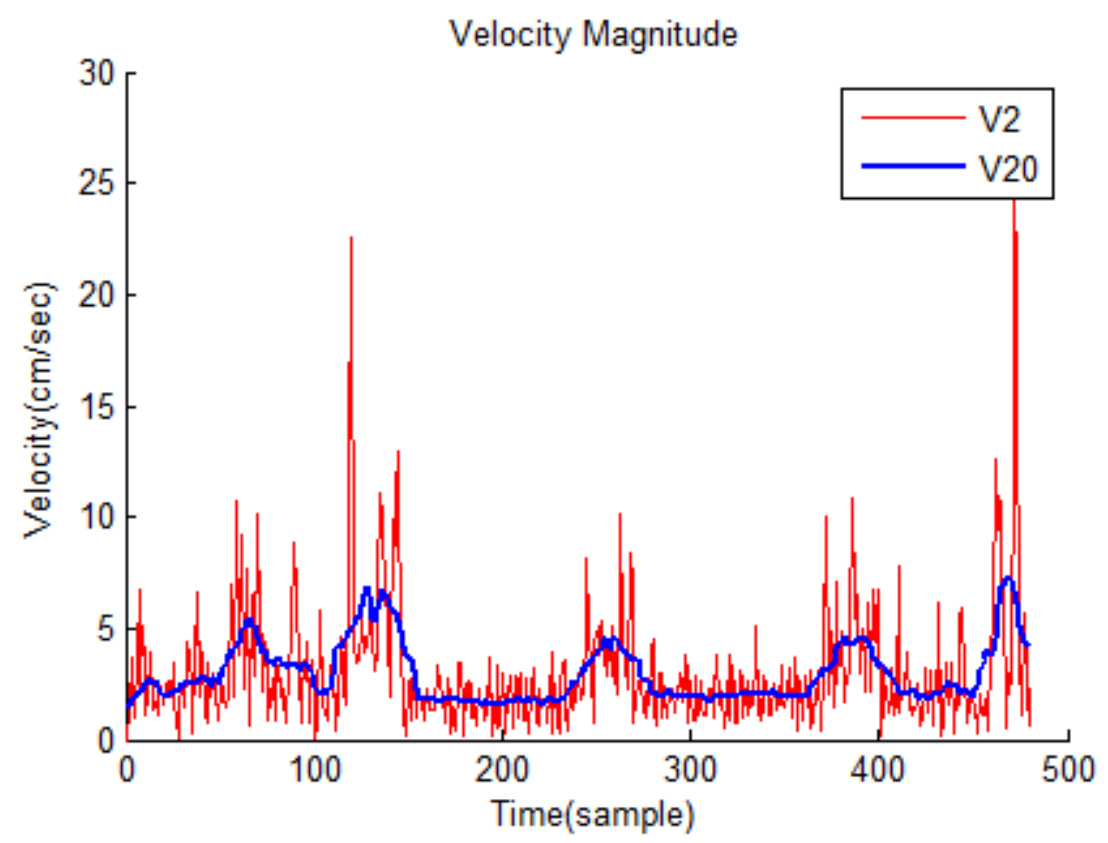

Figure 59: V2 and V20 comparison plot.

For comparing the change of velocity magnitude results, we put the plots from V2 and from V10 in Fig. 60, which show as a2 (red) and a10 (blue), and the plots from V2 and V20 in Fig. 61, which show as a2 (red) and a20 (blue). Figure 62 is a zoom-in plot of the beginning of Fig. 61 for observing the difference better where it has some amount of large fluctuations. From these graphs we can see that the change of velocity magnitude fluctuate dramatically. When the change of velocity magnitude value is above zero it means the speed of the subject is increasing and when below zero it means the speed of the subject is decreasing. 


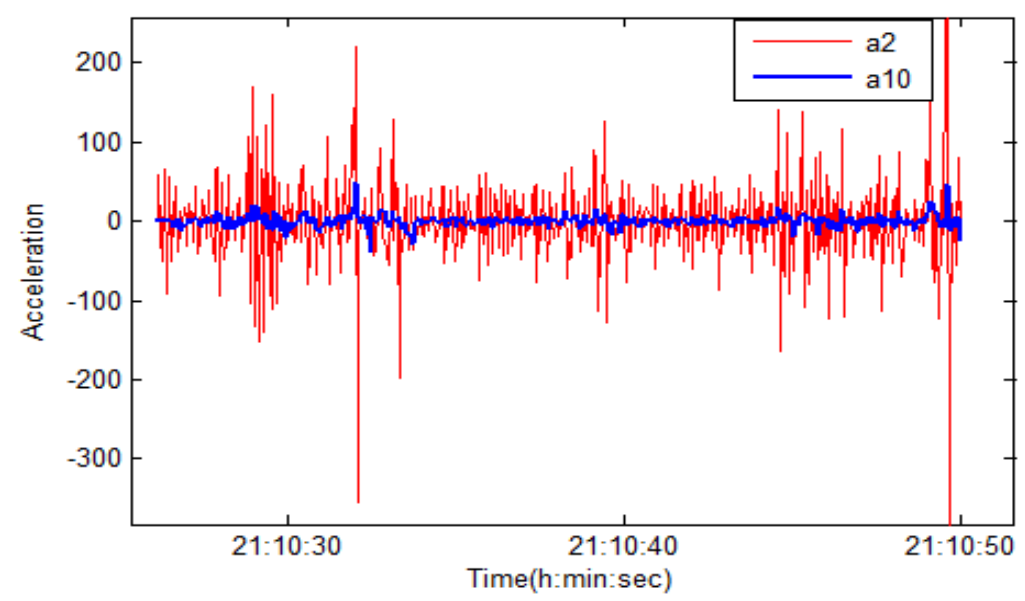

Figure 60: a2 and a10 comparison plot.

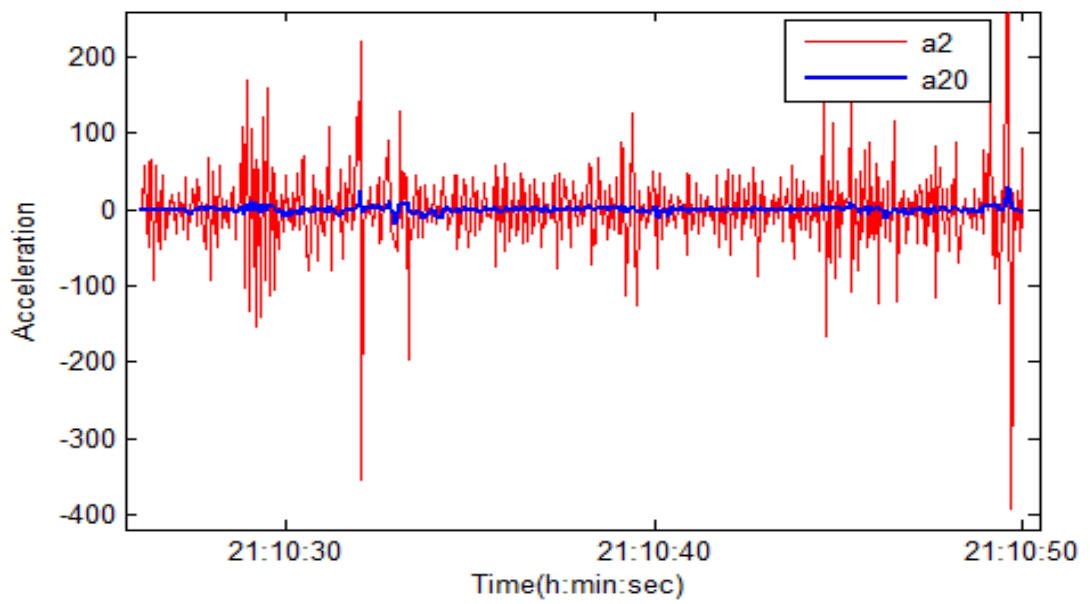

Figure 61: a2 and a20 comparison plot. 


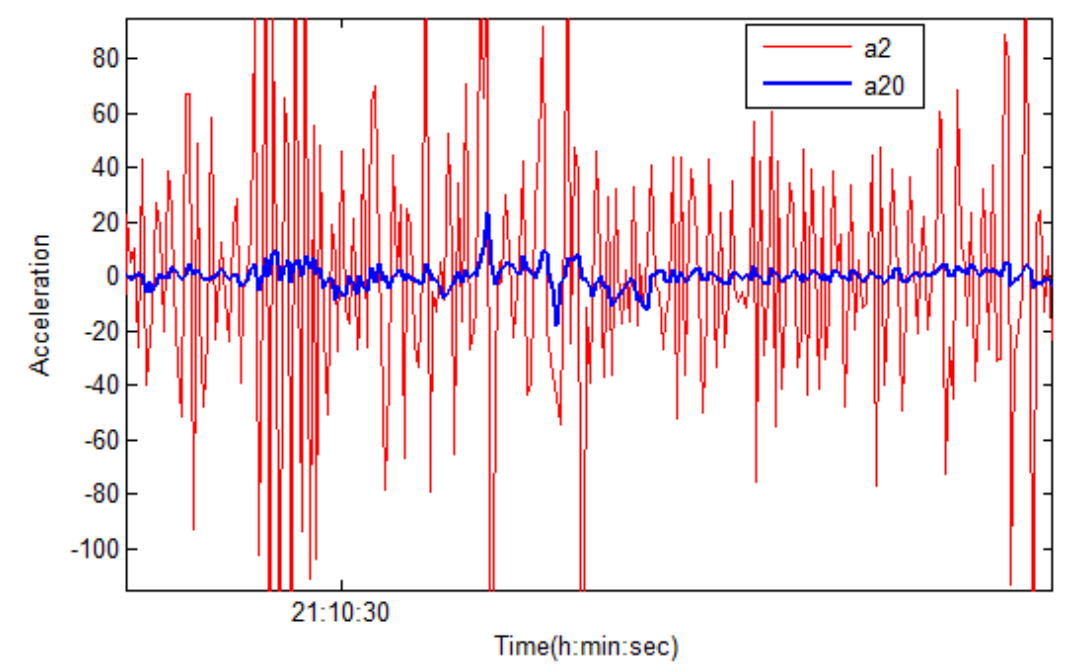

Figure 62: The zoom-in plot of the beginning of Figure 61.

\subsection{Discussion}

The method used to reduce noise is working well. The filtered velocity plot reduced some noise frequencies, so the plot looks much smoother. Zero phase filtering is able to smooth the plot and eliminate the noise. Using the moving average window is the most common way of doing noise reduction, so in this thesis the most of data processing used averaging window method to minimize the effect of noise.

The change of velocity magnitude algorithm was dependent on velocity magnitude calculation, so any noises within the velocity magnitude calculation was amplified within the computed the change of velocity magnitude data. When comparing the noises in Fig. 59 and Fig. 62, V2 and V20 velocity magnitude comparison and the change of velocity magnitude comparison respectively, that the noise in the velocity data was amplified significantly when used to produce the change of velocity magnitude plot. The V20 velocity magnitude and the change of velocity magnitude plots demonstrated a significant 
reduction in noise within the change of velocity magnitude data displaying a decrease in noise and as approximated by visual inspection, resulting in a reduction in computational error by 1 or more orders of magnitude. 


\section{Chapter 7:}

\section{Graphical User Interface}

This chapter will discuss the user interface for the graphical analysis design, functions of it and the usage of this interface.

\subsection{Introduction}

Graphical user interface (GUI) is an interface for computer users to quickly set and change the graphical output to observe various trends. This GUI works as a user friendly and time efficient method of analysing data. The features of participants data that clinicians would like to devote time to look at were discussed by clinicians in CIHR meeting. This GUI displays the features that were picked by clinicians. Section 7.2 discusses the design of this GUI that was created for graphical analysis for participant data files. Section 7.3 demonstrates the interface output plots and explains how to use it to get different features of pressure value data files.

\subsection{Design}


MATLAB was used to design this GUI for CIHR team members, doctors and other related people for a user friendly data processing of different features and participant specific constraints to monitor a participant's condition of a period of time.

This application was capable of displaying 5 characteristics list on the interface which are COP trajectory, velocity magnitude (show as 'Velocity'), the rate of change of velocity magnitude (show as 'Acceleration'), summing the pressure acting on every sensor (Sum Pres) and the number of active sensors (Act-Sen\#). One more push button on the interface was used for comparing plots from two different features and super-imposed them with two scaling systems, for example, comparison of velocity magnitude and the change of velocity magnitude (label as 'A\&V'). Data files were located in a scroll menu (Data files list). "Model file directory" was a browser to choose which directory's data file names want to be put under "Data files list". "HoldPlot" was added for comparing the different bed-exit transits of any one of the five features. To help distinguish the superimposed bed exit plots, a function was added to change plots' color and a Grid function was built to help people clearly see how much the difference was between postures transits. The GUI can be seen in Fig. 63. The interface contained all the basic functions also, zoom in, zoom out, and rotating the plot, etc.

This GUI helps doctors and researchers to observe trends associated with bed-exit of participants. The difference of bed-exit transfers from one patient or bed-exit transfers between several patients can be used to find out diverse health issues associated with bed mobility, the ability to live independently and health situation over time caused by various problems in their daily life. To prevent future potential injuries, care providers can be sent to intervene, based on abnormal bed mobility. 


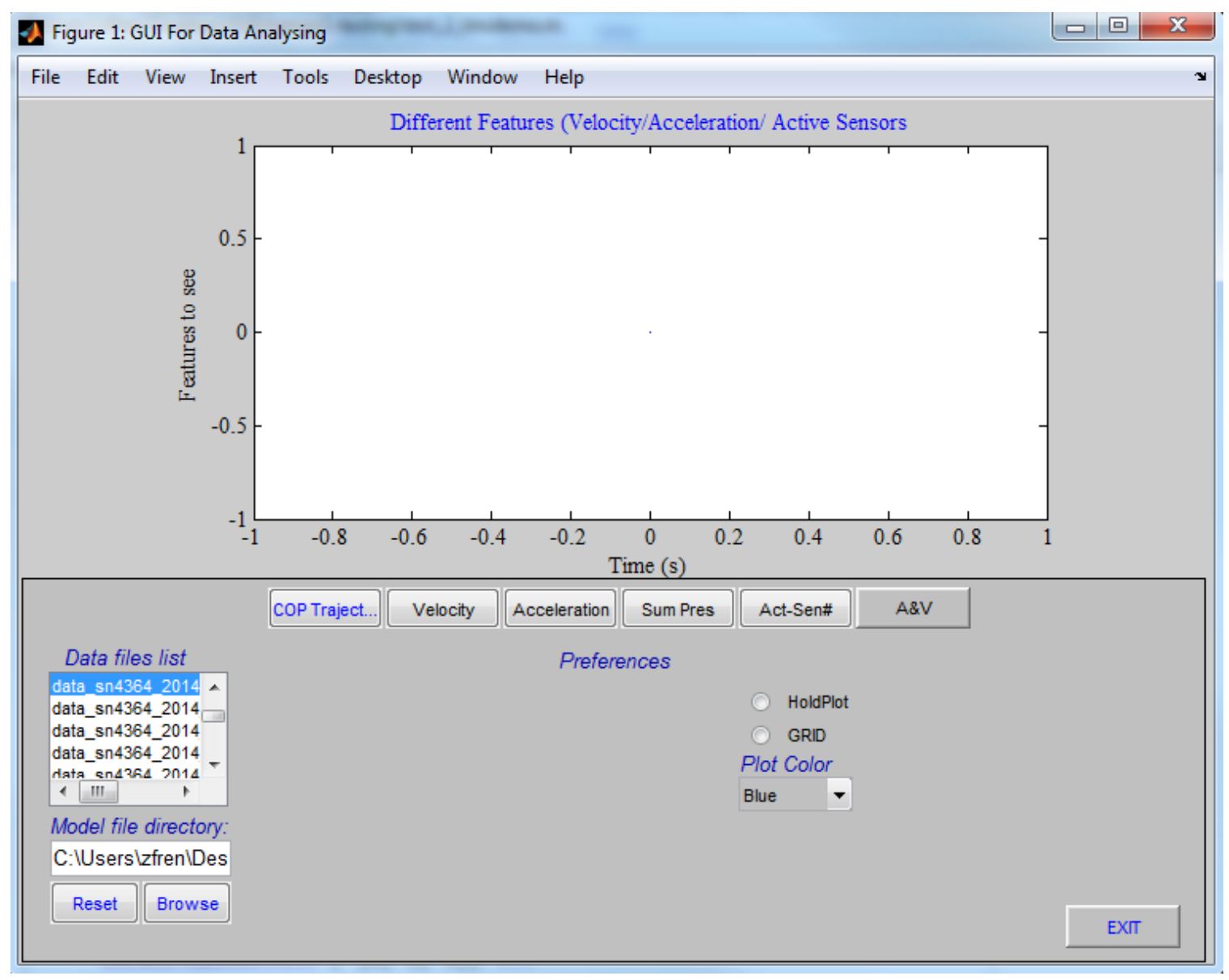

Figure 63: Graphical user interface.

\subsection{Usage of Interface}

A GUI program was written on MATLAB. Running the GUI from within MATLAB will display the interface as shown in Fig. 63. Based on the users' criteria, they chose one of the five features and the special "A\&V" button, and then an interface for picking a data file among all the data files would show up as Fig. 64. From all the directories pick one file to process. Figure 65 was an example of using this interface to do COP trajectory plotting. Comparing COP trajectory plots of two bed-exits data files at Fig. 66, two plots 
with different colors and grid function was on. Click on 'HoldPlot' to make plots over top of each other, and if we did not click on 'HoldPlot' again to release the function, the interface plots everything on the same graph. For changing next plot's color, click on 'PlotColor' and pick a color for next plot, color choices are shown in Fig. 67. Figure 67 included the color choices and the velocity plotted by using GUI. Figure 68 shows sum of pressure of one senior volunteer morning bed exit plot by using GUI, and Fig. 69 was the number of active sensors from the same morning bed exit as in Fig. 65. All the data files using in this chapter were real patients' uncontrolled data files recorded at their homes. Figure 70 had two line plots from two different features (velocity and the change of velocity magnitude) with two scaling systems. The left y axis showed the the change of velocity magnitude plot's scaling and the right y axis showed the velocity magnitude plot's scaling. Plotting two features into one plot for visual a comparison of different characteristics of a participant's bed-exit transfers.

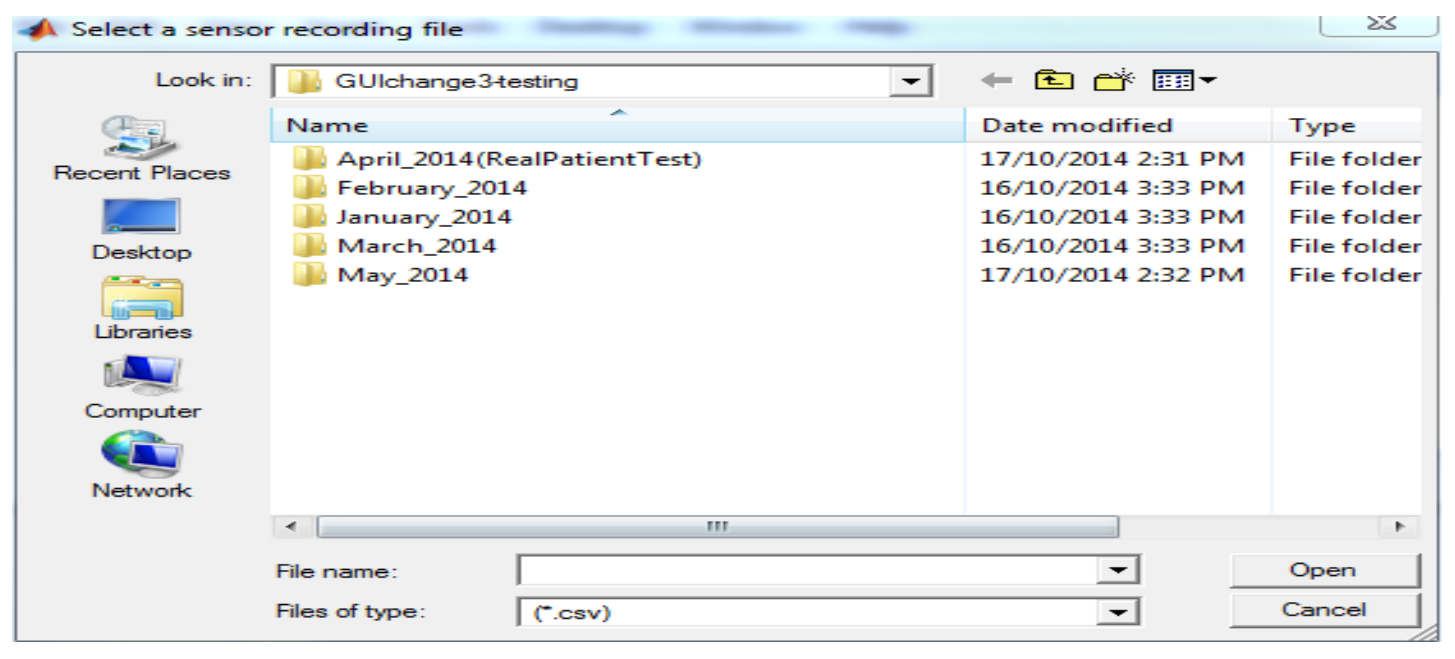

Figure 64: Picking data file interface. 


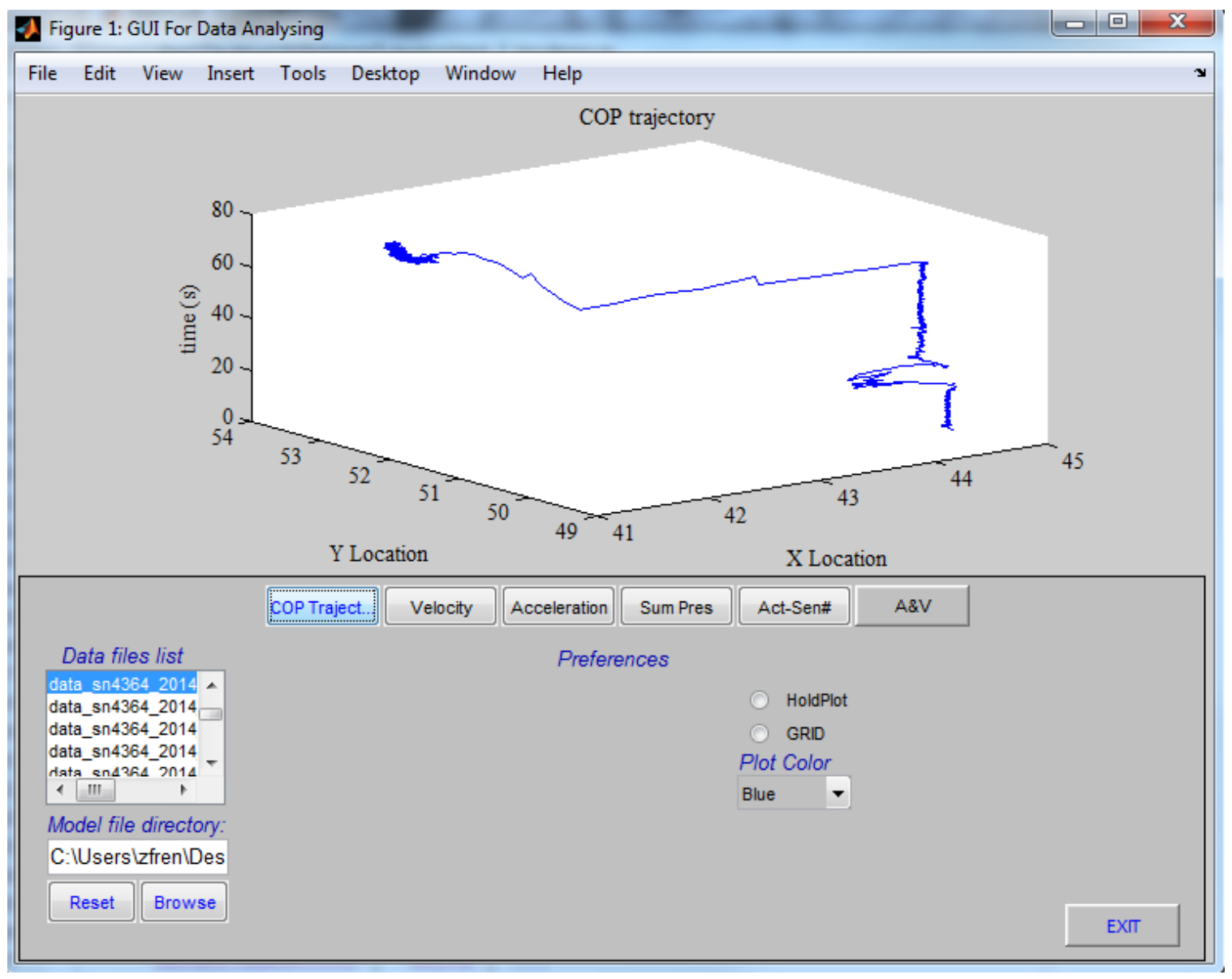

Figure 65: COP trajectory plot by using GUI. 


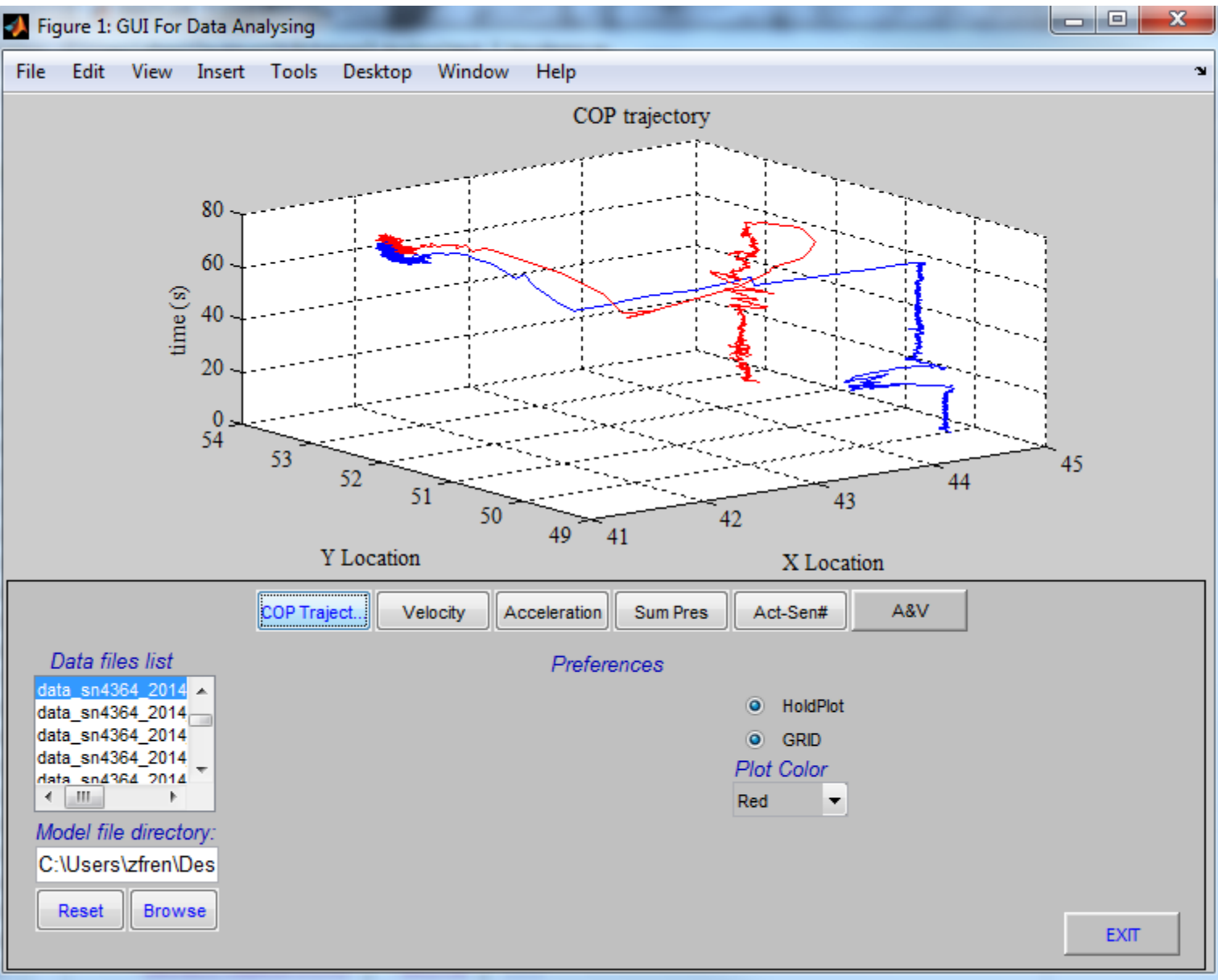

Figure 66: Comparison of two bed-exits on GUI. 


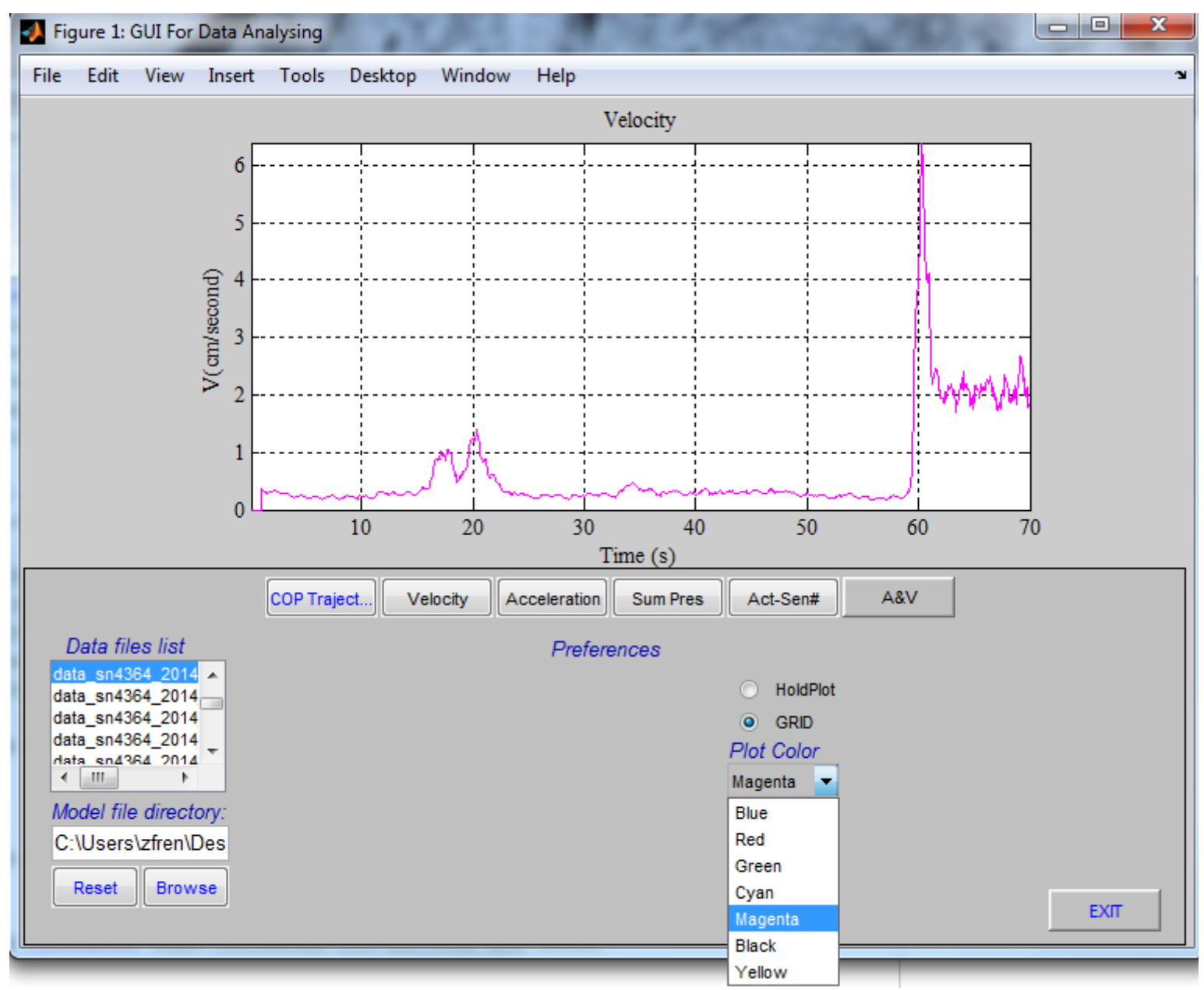

Figure 67: Choices of color for plots. 
Figure 1: GUI For Data Analysing

File Edit View Insert Tools Desktop Window Help

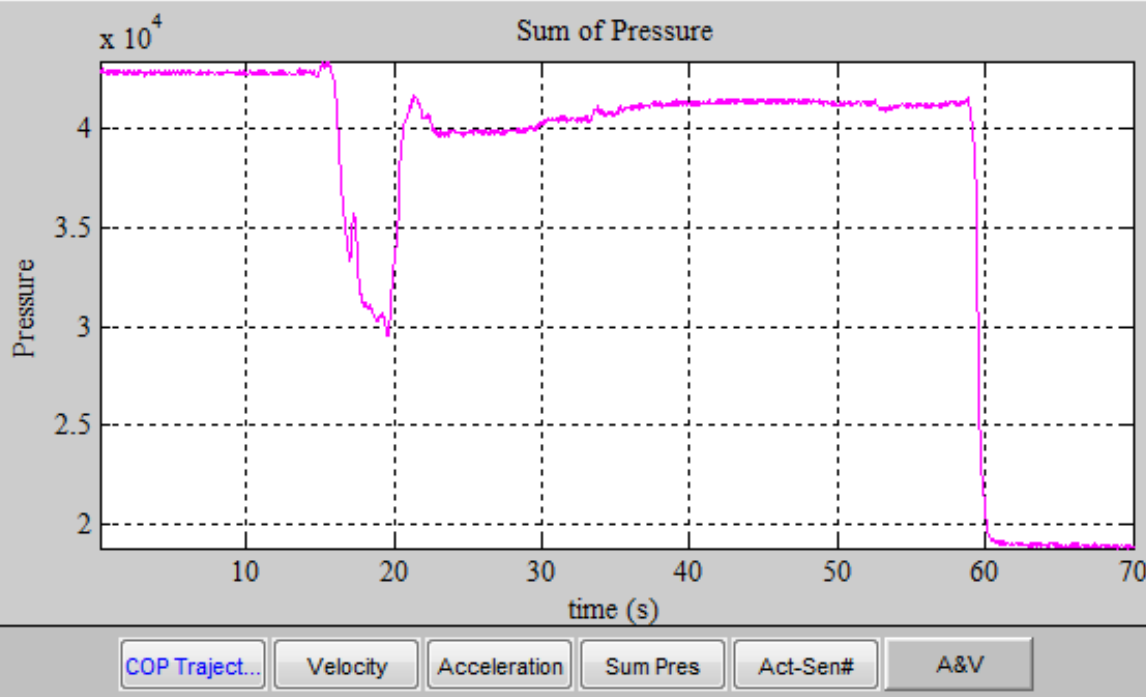

Data files list

data $\sin 43642014$ A

data_sn4364_2014

data_sn4364_2014

data_sn4364_2014

तata enA2GA 2014

1 III

Model file directory:

C:IUserslzfren\Des

Reset

Browse

Preferences

(1) HoldPlot

- GRID

Plot Color

Magenta

Figure 68: Sum of pressure changes during a bed-exit. 


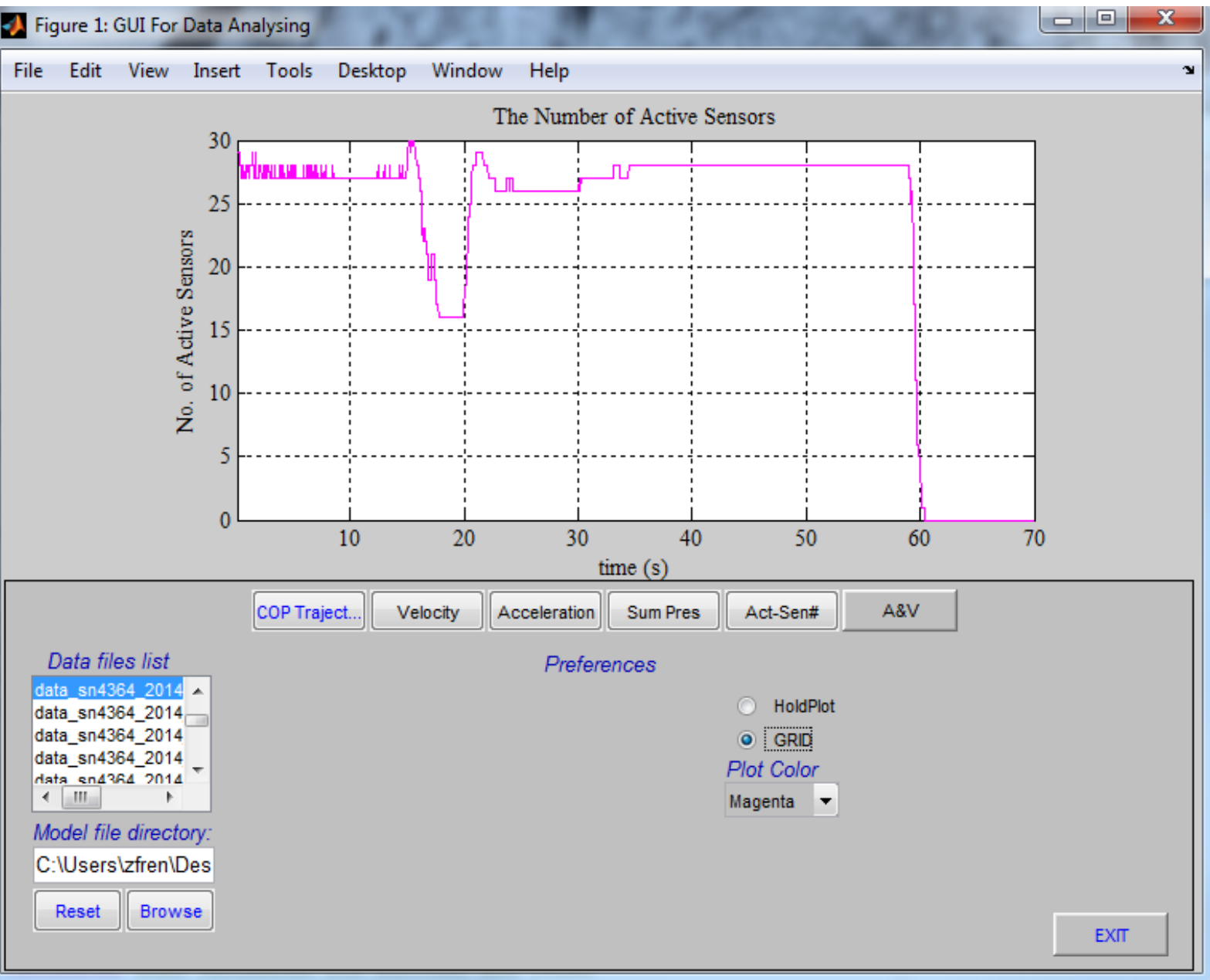

Figure 69: The number of active sensors during a bed-exit. 


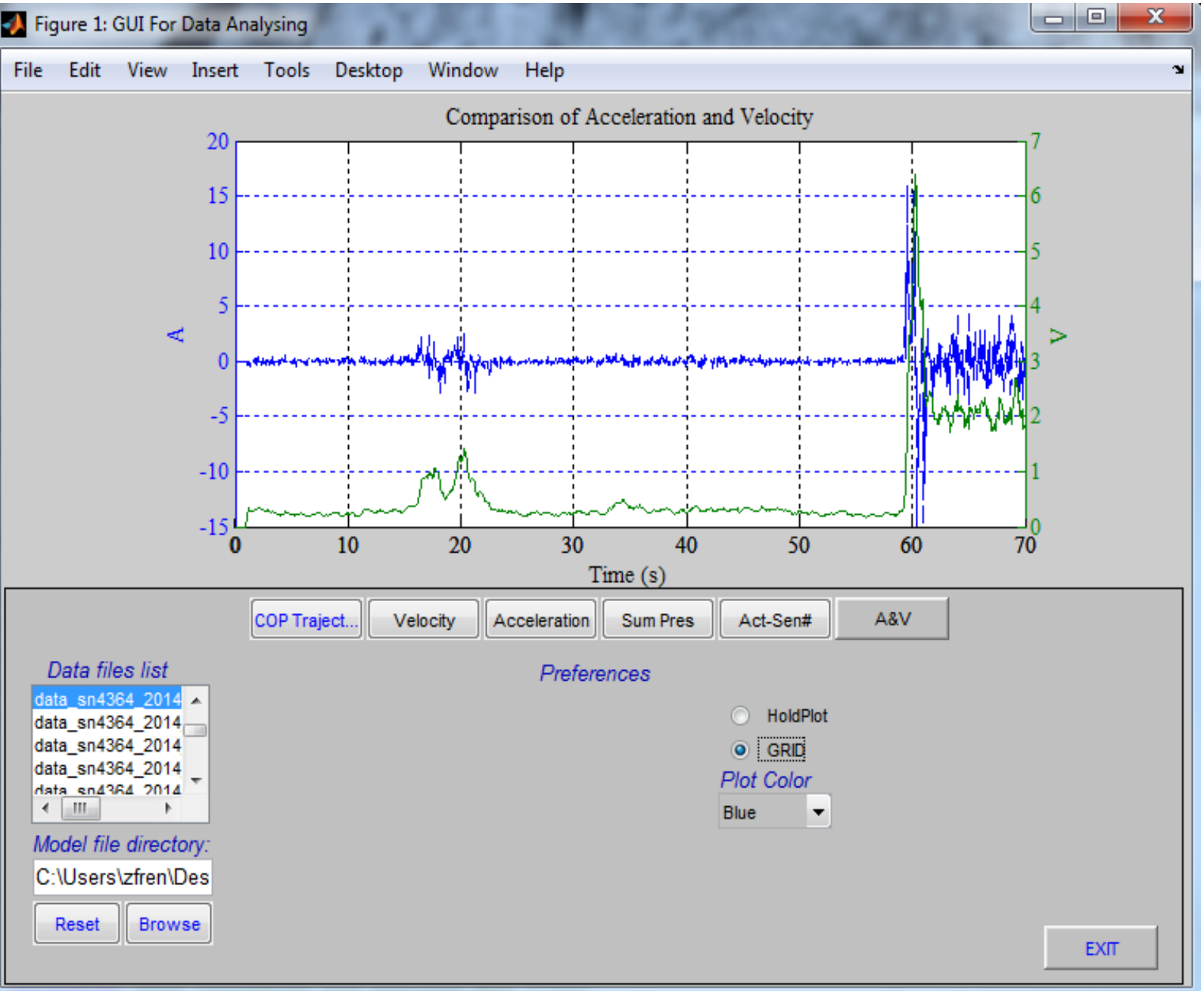

Figure 70: Comparison of Acceleration and velocity. 


\section{Chapter 8: Conclusion}

\subsection{Thesis Summary}

This thesis used a pressure-sensitive mat that is placed between a bed frame and the mattress. The pressure data can reveal important clinical information about the bed occupant. This thesis dealt with the analysis of bed exit characteristics in terms of centre of pressure trajectory and its dynamic behaviour. The algorithms were tested on data collected from bed occupants to demonstrate the various clinical features. A graphical user interface (GUI) was designed to assist health care providers in interpreting and comparing these clinical features. This work will be used to monitor frail older adults in their own homes to assist with early detection of mobility decline.

\subsection{Thesis Contributions}

Below is a list of contributions resulting from this research:

- An algorithm was designed and implemented to convert the data from different mat types and mat configurations into a common format that can be processed with a single set of algorithms.

- A set of algorithms were proposed to measure the Center of Pressure (COP) location and the average pressure magnitude. These algorithms plot the COP trajectory and generate a video of the COP trajectory over time. The movements 
of a subject on the bed, including the sways and rocks during bed-exit transfers can be seen in the COP trajectory plot and video. This set of algorithms finally extracts clinical health information of a subject's mobility such as smoothness, symmetry and stability, which could help in fall prevention.

- Several algorithms were implemented to compute and plot the COP dynamic behaviour. The COP speed and its rate of change also revealed additional information about an individual's smoothness and timing of movements. Filtering was used to reduce the noise.

- A Graphical User Interface (GUI) was designed to allow the user to select any desired transfers from any patient. The user could then plot and compare any of the following features: COP trajectory, speed and its variation, average pressure magnitude, and the total number of active sensors. This GUI was written to plot multiple, super-imposed graphs of the same feature as well as plot two different features with two different scaling factors in one graph.

- A number of approaches were proposed for noise deduction related to the COP dynamic behaviour.

- An algorithm was written to parse data files and find bed-exit times, truncate files, take every bed-exit transfer data out and put them into new CSV files. 


\subsection{Future Work}

Using non-intrusive pressure sensor mats to analyze the mobility of bed occupants can reveal important clinical information that can be crucial in the early detection of disease. In this thesis pressure mats were inserted in the middle of beds, which covered only parts of the beds. In the future, pressure mats covering the whole bed area can be used to get a better picture of how the subject moves on the bed instead of looking at part of the torso.

The algorithms that were designed in this thesis could be used to analyze data that are presently being collected from patients though longitudinal studies. Correlating the clinical information gathered from the pressure-sensitive mats with patient's health record will be valuable and will help optimize our algorithms. More volunteers will be recruited to collect in-home data for time ranges beyond one year so that more health problems will be captured, for instance, stroke and fall. If there are more falls happening to seniors, the data will be used to help for analysing fall pattern for future fall risk prediction. 


\section{References}

[1] “Canada - Total population 2014 | Statistic.” Statista. [Online]. Available: http://www.statista.com/statistics/263742/total-population-in-canada/. [Accessed: Nov. 29, 2014].

[2] S. D. C. Government of Canada. "Canadians in Context - Population Size and Growth / Indicators of Well-being in Canada." [Online]. Available: http://www4.hrsdc.gc.ca/.3ndic.1t.4r@-eng.jsp?iid=35, Sep. 12, 2006. [Accessed: Nov. 29, 2014].

[3] S. D. C. Government of Canada. "Canadians in Context - Aging Population / Indicators of Well-being in Canada." [Online]. Available: http://www4.hrsdc.gc.ca/.3ndic.1t.4r@-eng.jsp?iid=33, Sep. 12, 2006. [Accessed: Nov. 29, 2014].

[4] C. I. of H. R. Government of Canada. "Canadian Longitudinal Study on Aging (CLSA) - CIHR.” [Online]. Available: http://www.cihr-irsc.gc.ca/e/18542.html, Aug. 01, 2009. [Accessed: Nov. 29, 2014].

[5] P. H. A. of C. Government of Canada. "Changing Demographics, Aging and Health - The Chief Public Health Officer's Report on the State of Public Health in Canada, 2014: Public Health in the Future - Public Health Agency of Canada." [Online]. Available: http://www.phac-aspc.gc.ca/cphorsphc-respcacsp/2014/changeng.php\#a4, Sep. 08, 2014. [Accessed: Nov. 29, 2014]. 
[6] "Stroke/Transient Ischemic Attacks Symptoms - Diseases and Conditions - PDR Health." pdrhealth. [Online]. Available: http://www.pdrhealth.com/diseases/stroketransient-ischemic-attacks/symptoms. [Accessed: Nov. 29, 2014].

[7] "NIHSeniorHealth: Falls and Older Adults - Causes and Risk Factors." [Online]. Available: http://nihseniorhealth.gov/falls/causesandriskfactors/01.html. [Accessed: Nov. 29, 2014].

[8] C. I. of H. R. Government of Canada, "Reviews of health research - CIHR." [Online]. Available: http://www.cihr-irsc.gc.ca/e/45333.html, May 22, 2012. [Accessed: Nov. 29, 2014].

[9] M. Frischholz, L. Sarmento, M. Wenzel, K. Aquilina, R. Edwards and H.B. Coakham., "Telemetric Implantable Pressure Sensor for Short- and Long-Term Monitoring of Intracranial Pressure," in Proc. IEEE 29th Annual International Conference of the IEEE Engineering in Medicine and Biology Society (EMBC), 2007, pp. 514-514.

[10] M. Taylor, T. Grant, F. Knoefel, and R. Goubran, "Bed occupancy measurements using under mattress pressure sensors for long term monitoring of communitydwelling older adults," in Proc. 2013 IEEE International Symposium on Medical Measurements and Applications Proceedings (MeMeA), 2013, pp. 130-134.

[11] Z. Ren, T. Grant, R. Goubran, M. El-Tanany, F. Knoefel, H. Sveistrup, M. Bilodeau, and J. Jutai, "Analyzing center of pressure progression during bed exits," in Proc. IEEE 36th Annual International Conference of the IEEE Engineering in Medicine and Biology Society (EMBC), 2014, pp. 1786-1789. 
[12] F. Knoefel, Z. Ren, T. Grant, M. Bilodeau, R. Goubran, H. Sveistrup, and J. Jutai, "A method of analyzing bed exit movement in the home context," presented at Regional Geriatric Program of Eastern Ontario Annual General Meeting, Ottawa, Ontario, Canada, Oct. 24, 2014.

[13] S. Bennett, Z. Ren, R. Goubran, K. Rockwood, and F. Knoefel, “In-Bed Mobility Monitoring Using Pressure Sensors," submitted to 2014 IEEE Transactions on Instrument and Measurement, 2014.

[14] Y. Li, "Design of a Key Establishment Protocol for Smart Home Energy Management System," in Proc. 2013 Fifth International Conference on Computational Intelligence, Communication Systems and Networks (CICSyN), 2013, pp. 88-93.

[15] D. I. Townsend, R. Goubran, M. Frize, and F. Knoefel, "Preliminary results on the effect of sensor position on unobtrusive rollover detection for sleep monitoring in smart homes," in Proc. IEEE Annual International Conference of the IEEE Engineering in Medicine and Biology Society, 2009, pp. 6135-6138.

[16] J.-Y. Son, J.-H. Park, K.-D. Moon, and Y.-H. Lee, "Resource-aware smart home management system by constructing resource relation graph," IEEE Trans. Consum. Electron., vol. 57, no. 3, pp. 1112-1119, Aug. 2011.

[17] D.-M. Han and J.-H. Lim, “Smart home energy management system using IEEE 802.15.4 and zigbee," IEEE Trans. Consum. Electron., vol. 56, no. 3, pp. 14031410, Aug. 2010. 
[18] L. Jiang, D. Liu, and B. Yang, "Smart home research," in Proceedings of 2004 International Conference on Machine Learning and Cybernetics, 2004, vol. 2, pp. $659-663$.

[19] K. W. Schaie, Impact of Technology on Successful Aging. Springer Publishing Company, 2003.

[20] A. Dunne, Hertzian Tales: Electronic Products, Aesthetic Experience, and Critical Design. The MIT Press, 2008.

[21] S. Livingstone, Young People and New Media: Childhood and the Changing Media Environment. SAGE, 2002.

[22] S. Gouthaman, A. Pandya, O. Karande, and D. R. Kalbande, "Gesture detection system using smart watch based motion sensors," in Proc. 2014 International Conference on Circuits, Systems, Communication and Information Technology Applications (CSCITA), 2014, pp. 311-316.

[23] T. Bialy, J. Kobusinski, M. Malecki, and K. Stefaniak, "EMeH: Extensible mobile platform for healthcare," in Proc. 2011 Federated Conference on Computer Science and Information Systems (FedCSIS), 2011, pp. 355-361.

[24] A. J. Saldarriaga, J. J. Pérez, J. Restrepo, and J. Bustamante, “A mobile application for ambulatory electrocardiographic monitoring in clinical and domestic environments," in Health Care Exchanges (PAHCE), 2013 Pan American, 2013, pp. $1-4$.

[25] J. Kim, S. Kim, S. Park, and J. Hong, "Home appliances controlling through Smart TV set-top box with screen-mirroring remote controller," in Proc. 2013 International Conference on ICT Convergence (ICTC), 2013, pp. 1009-1012. 
[26] A. Zhu, P. Lin, S. Cheng, "Design and Realization of Home Appliances Control System Based on the Android Smartphone," in Proc. Control Engineering and Communication Technology (ICCECT), 2012 International Conference, 2012, pp. $56-59$.

[27] D. He, R. Xia, W. Lin, and T. G. Habetler, “A survey on consumer electronic products in residential home for demand response," in Proc. IEEE 38th Annual Conference on IEEE Industrial Electronics Society, 2012, pp. 4816-4820.

[28] S. Wang, M. Skubic, and Y. Zhu, "Activity Density Map Visualization and Dissimilarity Comparison for Eldercare Monitoring," IEEE Trans. Inf. Technol. Biomed., vol. 16, no. 4, pp. 607-614, Jul. 2012.

[29] A. Arcelus, M. Holtzman, R. Goubran, H. Sveistrup, P. Guitard, and F. Knoefel, "Analysis of commode grab bar usage for the monitoring of older adults in the smart home environment," in Proc. IEEE 31th Annual International Conference of the IEEE Engineering in Medicine and Biology Society (EMBC), 2009, pp. 6155-6158.

[30] V. Joshi, M. Holtzman, A. Arcelus, R. Goubran, and F. Knoefel, "Highly survivable bed pressure mat remote patient monitoring system for mHealth," in Proc. IEEE 34th Annual International Conference of the IEEE Engineering in Medicine and Biology Society (EMBC), 2012, pp. 268-271.

[31] J. Leon and T. J. Lair, "Functional Status of the Noninstitutionalized Elderly: Estimates of ADL and IADL Difficulties," Agency for Health Care Policy and Reserach, Rockville, MD, PHS 90-3462,1990. 
[32] A. Arcelus, C. L. Herry, R. A. Goubran, F. Knoefel, H. Sveistrup, and M. Bilodeau, "Determination of Sit-to-Stand Transfer Duration Using Bed and Floor Pressure Sequences,” IEEE Trans. Biomed. Eng., Oct. 2009, vol. 56, no. 10, pp. 2485-2492.

[33] I. Veledar, A. Arcelus, R. Goubran, F. Knoefel, H. Sveistrup, and M. Bilodeau, "Sitto-stand timing measurements using pressure sensitive technology," in Proc. IEEE Instrumentation and Measurement Technology Conference (I2MTC), 2010, pp. $1337-1340$.

[34] J. H. Porter, “Differential fiber optic proximity sensor,” US4358960 A, Nov. 16, 1982.

[35] M. Zimmerman, L. Felton, E. Lacsamana, and R. Navarro, "Next generation low stress plastic cavity package for sensor applications," in Electronic Packaging Technology Conference (EPTC). Proceedings of $7^{\text {th }}$, Dec. 2005, vol. 1, pp. 7 pp.231-237.

[36] G. Reyes, D. Wang, R. Nair, C. Li, X. Li, and J. Lin, "VitalTrack: A Doppler radar sensor platform for monitoring activity levels," in 2012 IEEE Topical Conference on Biomedical Wireless Technologies, Networks, and Sensing Systems (BioWireleSS), 2012, pp. 29-32.

[37] G. Wang, S. Rodriguez, C. Gu, J. Lin, and C. Li, "Non-contact measurement of rotational movement using miniature Doppler radar," in Proc. IEEE Wireless and Microwave Technology Conference (WAMICON), 2012 IEEE 13th Annual, 2012, pp. 1-6. 
[38] Y. Li, K. C. Ho, and M. Popescu, "Efficient Source Separation Algorithms for Acoustic Fall Detection Using a Microsoft Kinect," IEEE Trans. Biomed. Eng., vol. 61, no. 3, pp. 745-755, Mar. 2014.

[39] M. Parajuli, D. Tran, W. Ma, and D. Sharma, "Senior health monitoring using Kinect," in Proc. 2012 Fourth International Conference on Communications and Electronics (ICCE), 2012, pp. 309-312.

[40] D. Townsend, R. Goubran, and F. Knoefel, "Amplitude-based central apnea screening," in Proc. IEEE 33th International Workshop on Medical Measurements and Applications Proceedings (MeMeA), 2011, pp. 395-398.

[41] A. Arcelus, I. Veledar, R. Goubran, F. Knoefel, H. Sveistrup, and M. Bilodeau, "Measurements of Sit-to-Stand Timing and Symmetry From Bed Pressure Sensors," IEEE Trans. Instrum. Meas., vol. 60, no. 5, pp. 1732-1740, May 2011.

[42] A. Arcelus, R. Goubran, F. Knoefel, H. Sveistrup, and M. Bilodeau, "Detection of bouncing during sit-to-stand transfers with sequential pressure images," in Proc. IEEE 33th International Workshop on Medical Measurements and Applications Proceedings (MeMeA), 2011, pp. 158-161.

[43] A. M. Adami, M. Pavel, T. L. Hayes, A. G. Adami, and C. Singer, "A method for classification of movements in bed," in Proc. IEEE 33th Annual International Conference of the IEEE Engineering in Medicine and Biology Society (EMBC), 2011, pp. 7881-7884.

[44] D. Townsend, R. Goubran, F. Knoefel, and J. Leech, "Validation of Unobtrusive Pressure Sensor Array for Central Sleep Apnea Screening," IEEE Trans. Instrum. Meas., vol. 61, no. 7, pp. 1857-1865, Jul. 2012. 
[45] D. Townsend, M. Holtzman, R. Goubran, M. Frize, and F. Knoefel, "Relative Thresholding With Under-Mattress Pressure Sensors to Detect Central Apnea," IEEE Trans. Instrum. Meas., vol. 60, no. 10, pp. 3281-3289, Oct. 2011.

[46] D. I. Townsend, M. Holtzman, R. Goubran, M. Frize, and F. Knoefel, "Measurement of Torso Movement With Delay Mapping Using an Unobtrusive Pressure-Sensor Array," IEEE Trans. Instrum. Meas., vol. 60, no. 5, pp. 1751-1760, May 2011.

[47] M. Taylor, R. Goubran, and F. Knoefel, "Patient standing stability measurements using pressure sensitive floor sensors," in Proc. IEEE 2012 Instrumentation and Measurement Technology Conference (I2MTC), 2012, pp. 1275-1279.

[48] M. Holtzman, D. Townsend, R. Goubran, and F. Knoefel, "Breathing sensor selection during movement," in Proc. IEEE 33th Annual International Conference of the IEEE Engineering in Medicine and Biology Society (EMBC), 2011, pp. 381384.

[49] M. Holtzman, R. Goubran, F. Knoefel, and J. Pereira, "Measurement of respiratory changes in palliative care," in Proc. IEEE 2012 Instrumentation and Measurement Technology Conference (I2MTC), 2012, pp. 1472-1476.

[50] M. Holtzman, R. Goubran, and F. Knoefel, "Maximal ratio combining for respiratory effort extraction from pressure sensor arrays," in Proc. IEEE 2011 IEEE International Workshop on Medical Measurements and Applications Proceedings (MeMeA), 2011, pp. 88-92.

[51] N. Foubert, A. M. McKee, R. A. Goubran, and F. Knoefel, "Lying and sitting posture recognition and transition detection using a pressure sensor array," in Proc. 
IEEE 2012 IEEE International Symposium on Medical Measurements and Applications Proceedings (MeMeA), 2012, pp. 1-6.

[52] S. L. Bennett, R. Goubran, K. Rockwood, A. Arcelus, and F. Knoefel, "Pressure signal feature extraction for the differentiation of clinical mobility assessments," in Proc. IEEE 2012 IEEE International Symposium on Medical Measurements and Applications Proceedings (MeMeA), 2012, pp. 1-5.

[53] S. Bennett, R. Goubran, K. Rockwood, and F. Knoefel, "Automated assessment of mobility in bedridden patients," in Proc. IEEE 35th Annual International Conference of the IEEE Engineering in Medicine and Biology Society (EMBC), 2013, pp. 4271-4274.

[54] S. L. Bennett, R. Goubran, K. Rockwood, and F. Knoefel, "Distinguishing between stable and unstable sit-to-stand transfers using pressure sensors," in Proc. IEEE 2014 International Symposium on Medical Measurements and Applications (MeMeA), 2014, pp. 1-4.

[55] S. L. Bennett, R. Goubran, K. Rockwood, and F. Knoefel, "Monitoring the relief of pressure points for pressure ulcer prevention: A subject dependent approach," in Proc. IEEE 2013 International Symposium on Medical Measurements and Applications Proceedings (MeMeA), 2013, pp. 135-138.

[56] M. Holtzman, R. Goubran, and F. Knoefel, "Motion monitoring in palliative care using unobtrusive bed sensors," in Proc. IEEE 36th Annual International Conference of the IEEE Engineering in Medicine and Biology Society (EMBC), 2014, pp. 5760-5763. 
[57] A. Arcelus, "Context-Aware Smart Home Monitoring Through the Analysis of Pressure Sequences,” Ph.D. dissertation, Dept. Elect. Comput. Eng., Carleton University, Ottawa, ON, 2010.

[58] A. Arcelus, R. Goubran, H. Sveistrup, M. Bilodeau, and F. Knoefel, "Context-aware smart home monitoring through pressure measurement sequences," in Proc. IEEE 2010 International Workshop on Medical Measurements and Applications Proceedings (MeMeA), 2010, pp. 32-37.

[59] M. Taylor, "Image Processing Algorithms for Patient Mobility Monitoring using Pressure Sensitive Bed and Floor Arrays,” M.A.Sc. thesis, Dept. Biomedical Eng., Carleton University, Ottawa, ON, 2012.

[60] M. Taylor, D. McEwen, R. Goubran, H. Finestone, F. Knoefel, H. Sveistrup, and M. Bilodeau, "Assessing standing stability of older adults using pressure sensitive arrays," in Proc. IEEE 2012 International Symposium on Medical Measurements and Applications Proceedings (MeMeA), 2012, pp. 1-5.

[61] F. Knoefel, M. Taylor, T. Grant, S. Rocan, J. Jutai, H. Sveistrup, R. Goubran, J. Taylor, M. Bilodeau, "Unobtrusive Home Bed Transfer Monitoring in an Older Adult," presented at the 41st Annual Scientific and Educational Meeting Canadian Association on Gerontology, Vancouver, Canada, 2012.

[62] M. P. Murray, A. Seireg, and R. C. Scholz, "Center of gravity, center of pressure, and supportive forces during human activities.," J. Appl. Physiol., vol. 23, no. 6, pp. 831-838, Dec. 1967.

[63] X. Tortolero, K. Masani, T. A. Thrasher, and M. R. Popovic, "Instability Prediction by Monitoring Center of Pressure During Standing," in Proc. IEEE 28th Annual 
International Conference of the IEEE Engineering in Medicine and Biology $\operatorname{Society}(E M B C), 2006$, pp. 5412-5415.

[64] J. Fiolka and Z. Kidon, "Center of pressure trajectory segmentation for postural stability analysis," in Proc. Mixed Design of Integrated Circuits and Systems (MIXDES), 2011 Proceedings of the 18th International Conference, 2011, pp. 3943.

[65] P. Era, P. Sainio, S. Koskinen, P. Haavisto, M. Vaara, and A. Aromaa, "Postural balance in a random sample of 7,979 subjects aged 30 years and over," Gerontology, vol. 52, no. 4, pp. 204-213, 2006.

[66] M. E. Tinetti, M. Speechley, and S. F. Ginter, "Risk factors for falls among elderly persons living in the community," N. Engl. J. Med., vol. 319, no. 26, pp. 17011707, Dec. 1988.

[67] F. Mourey, A. Grishin, P. d' Athis, T. Pozzo, and P. Stapley, "Standing up from a chair as a dynamic equilibrium task: a comparison between young and elderly subjects," J. Gerontol. A. Biol. Sci. Med. Sci., vol. 55, no. 9, pp. B425-431, Sep. 2000.

[68] A. M. S. Baldan, S. R. Alouche, I. M. G. Araujo, and S. M. S. F. Freitas, "Effect of light touch on postural sway in individuals with balance problems: a systematic review," Gait Posture, vol. 40, no. 1, pp. 1-10, May 2014.

[69] E. Versi, “'Gold standard' is an appropriate term.,” BMJ, vol. 305, no. 6846, pp. 187, Jul. 1992.

[70] D. Halliday, J. Walker, R. Resnick, Fundamentals of Physics, Chapters 33-37. 2010. 
[71] D. Townsend, "Context-aware algorithms for sleep apnea monitoring and sensor acceptance using unobtrusive pressure sensors arrays," Ph.D. thesis, Dept. of Elect. and Comput. Eng., Carleton University, Ottawa, ON, Canada, 2012.

[72] A.V. Oppenheim, and R.W. Schafer, Discrete-Time Signal Processing. PrenticeHall, 1989. 